\title{
Spatially varying fuzzy multi-scale uncertainty propagation in unidirectional fibre reinforced composites
}

\author{
Susmita Naskar ${ }^{\mathrm{a}}$, Tanmoy Mukhopadhyay ${ }^{\mathrm{b},{ }^{*},}$, Srinivas Sriramula ${ }^{\mathrm{a}}$ \\ ${ }^{a}$ School of Engineering, University of Aberdeen, Aberdeen, UK \\ ${ }^{b}$ Department of Engineering Science, University of Oxford, Oxford, UK \\ *Email address: tanmoy.mukhopadhyay@eng.ox.ac.uk (T. Mukhopadhyay)
}

\begin{abstract}
This article presents a non-probabilistic fuzzy based multi-scale uncertainty propagation framework for studying the dynamic and stability characteristics of composite laminates with spatially varying system properties. Most of the studies concerning the uncertainty quantification of composites rely on probabilistic analyses, where the prerequisite is to have the statistical distribution of stochastic input parameters. In many engineering problems, these statistical distributions remain unavailable due to the restriction of performing large number of experiments. In such situations, a fuzzy-based approach could be appropriate to characterize the effect of uncertainty. A novel concept of fuzzy representative volume element (FRVE) is developed here for accounting the spatially varying non-probabilistic source-uncertainties at the input level. Such approach of uncertainty modelling is physically more relevant than the prevalent way of modelling non-probabilistic uncertainty without considering the ply-level spatial variability. An efficient radial basis function based stochastic algorithm coupled with the fuzzy finite element model of composites is developed for the multi-scale uncertainty propagation involving multi-synchronous triggering parameters. The concept of a fuzzy factor of safety (FFoS) is discussed in this paper for evaluation of safety factor in the non-probabilistic regime. The results reveal that the present physically relevant approach of modelling fuzzy uncertainty considering plylevel spatial variability obtains significantly lower fuzzy bounds of the global responses compared to the conventional approach of non-probabilistic modelling neglecting the spatially varying attributes.
\end{abstract}

Keywords: composite laminates; spatially varying fuzzy uncertainty; fuzzy representative volume element; fuzzy multi-scale analysis; radial basis function 


\section{Introduction}

Composite materials are increasingly being utilised as various components of aerospace, civil, automobile, mechanical and sports structures due to their high strength-to-weight ratio and cost effectiveness. Composites materials can also be aero-elastically customized to meet different design conditions. Even though laminated composite structures have the advantage of modulating large number of design parameters to achieve various application-specific requirements, this concurrently brings the challenge of manufacturing the structure according to exact design specifications. Largescale production of such structures according to the requirements of industry is often subjected to large amount of variability arising from unavoidable manufacturing imperfections (such as intralaminate voids and excess matrix voids, excess resin between plies, incomplete curing of resin, porosity, variations in lamina thickness and fibre properties), lack of experiences and complexity of the structural configuration [Dey et al. (2018a)]. The issue aggravates further due to uncertain operational and environmental factors and the possibility of incurring different forms of damages and defects during the service life. The structural performances of composite materials are often subjected to significant element of risks due to being susceptible to multiple uncertainties. Hence it is important to quantify the effect of source-uncertainties for composite structures, so that an inclusive design paradigm could be adopted to avoid any compromise in the aspects of safety and serviceability.

Deterministic analyses of composite structures have received considerable attention from the scientific community [Reddy (2003), Chakrabarti et al. (2013), Liew (1996), Liew and Huang (2003), Neves and Ferreira (2016), Tornabene et al. $(2017,2018)]$. The aspect of considering uncertainty in the analysis and design of composite structures are becoming increasingly recognised in last few years. Probabilistic approaches are found to be predominantly used to quantify the effect of uncertainty in composite structures following intrusive [Lal and Singh (2010), Scarth and Adhikari (2017), Zhou et al. (2017)] as well as non-intrusive [Shaw et al. (2010), Dey et al. (2016a, 2018), Umesh and Ganguli (2013), Naskar et al. (2017a, 2017b), Karsh et al. (2018), Sakata et al. (2008), Naskar and Sriramula (2017a, 2017b, 2017c, 2018), Mukhopadhyay and Adkhikari (2016b); Mukhopadhyay et al. (2016c)] methods. The prerequisite of carrying out a probabilistic analysis is to 


\section{Fuzzy multi-scale uncertainty propagation}

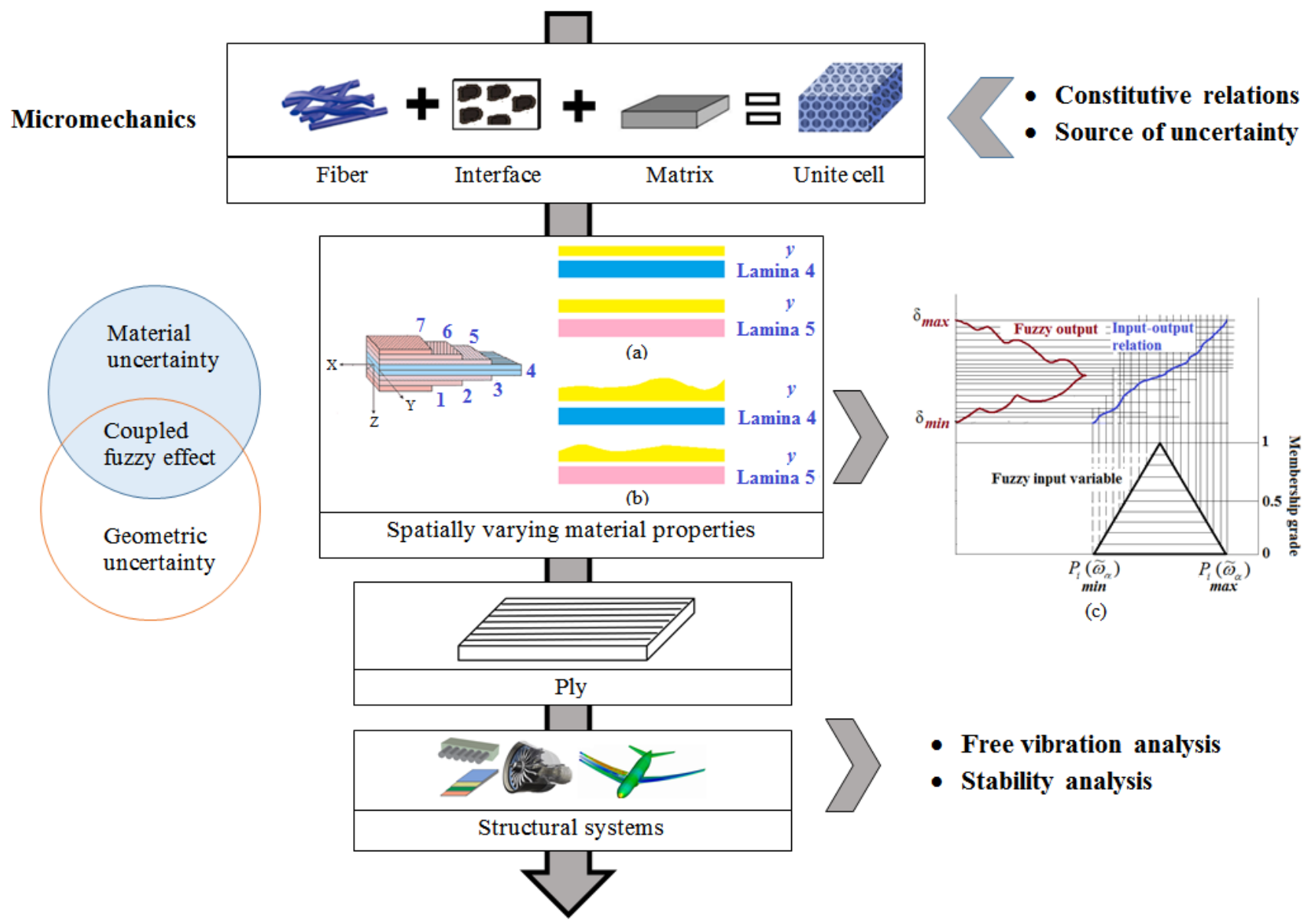

Fig. 1 Fuzzy multi-scale analysis of composite laminates considering the coupled effect of material and geometric uncertainty (a) Typical distribution of a typical material property $y$ (such as $E_{1}$ ) along a cross-sectional view (X-Z plane) of two laminae for a particular realization in case of the conventional approach where spatial variation of $y$ is ignored (b) Typical distribution of a material property $y$ along a cross-sectional view (X-Z plane) of two laminae for a particular realization in case of the present approach considering spatial variation of material properties (c) Typical representation of a fuzzy based analysis

have the statistical distribution of stochastic input parameters available. Composites being a multilayerd complex and expensive material, statistical distributions of the stochastic input parameters may not always be available due to restriction of performing large number of experimnents to characterize the material properties. In such situation, a non-probabilistic approach (such as fuzzy uncertainty) could be suitable to characterize the effect of uncertainty. The fuzzy based uncertainty propagation approach is found to be adopted in very limited number of studies concerning composites [Dey et al. (2016b), Pawar et al. (2012), Pawar and Ganguli (2007), Muc and Kedziora (2001)]. However, these studies do not consider the inevitable spatial variability in material properities of each of the laminas. This approach of modelling the non-probabilistic form of 
uncertainty without accounting spatial variability has limited practical relevance. Thus in this paper, we aim to consider the ply-level spatial variability of the material properties of composites in a more realistic non-probabilistic framework (refer to figure 1). The aspect of spatial variation of lamina properties is illustrated in figure 1(b) for a particular realization (i.e. a typical sample of the Monte Carlo simulation), wherein it can be noticed that the stochastic attributes vary in the x-y plane as well as along the z-axis (i.e. for different laminae).

In general, the fuzzy finite element analysis [Lei and Chen (2002), Mace et al. (2005)] can couple the potential of finite element method and uncertainty modeling capability of the fuzzy approach [Zadeh (1975)] in the presence of sparse input data. A way of viewing the fuzzy inputoutput parameters is the universality of an interval variable. In the interval approach, the uncertainties of input parameters are expressed by interval values where a statement about the likelihood of the individual values within the interval is not given. In order to solve the corresponding finite element system, the interval arithmetic technique may be used. In the fuzzy finite element method, input variables are characterized by fuzzy sets to represent data and information possessing non-statistical uncertainties. The underlying principle of fuzzy representation is that the boundary values are generally less possible and therefore have a lower likelihood compared to the expected value. In this way, the fuzzy finite element based method provides a generalization of the interval finite element method using multiple $\alpha$-cuts. It can be noted in this context that the intervals do not represent the numerical values of a parameter, but a knowledge regarding the range of possible values (lower and upper bounds) that the parameter can possess. In fuzzy approach, a membership function is introduced to generalize the interval based approach.

The propagation of uncertainty in composite structures possesses significant computational challenges as the evaluation of structural responses (i.e. output) for composites normally involves a complicated and computationally expensive numerical process like finite element method. This paper develops a radial basis function (RBF) [Beatson (1999), Fasshauer (1997), Hon and Mao (2001), Kansa (1990a, 1990b), Kansa and Hon (2000)] based non-probabilistic uncertainty propagation method coupled with the fuzzy finite element model of composites to investigate the dynamics and 
stability characteristics of the structure in an efficient manner. In the fuzzy RBF method, the RBF model acts as an emulator (surrogate) for the expensive finite element model at the zero level membership function. The input-output domain corresponding to the zero level membership function is mapped using the RBF based metamodel formed on the basis of Sobol's sequence [Sobol (1967)]. Thereby, the RBF models can be used for prediction corresponding to the higher $\alpha$-levels of fuzzy membership function. To incorporate the RBF based approach in fuzzy analysis, the interval variables corresponding to each alpha-cut can be treated as a random variable with uniformly distributed probability distribution function. In the possibilistic interpretation of uncertainty [(Denga et al. (2012), Möller and Beer, (2004)], the fuzzy variables can be thought as generalized interval variables, allowing one to use the techniques intended for the interval analysis such as classical interval arithmetic [Moore (1966)], affine analysis [Degrauwe et al. (2010)] or vertex theorems [Qiu et al. (2005)]. Fuzzy based uncertainty quantification is found to be adopted in various engineering analyses [Babuška and Silva (2014), Rao and Annamdas (2008), Díaz-Madroñero et al. (2004), Chandrashekhar and Ganguli (2009), Chowdhury and Adhikari (2012), Altmann et al. (2012)].

A careful review of the literature concerning the uncertainty analysis of composites reveals that the effect of spatially varying non-probabilistic uncertainty has not been addressed so far. Moreover, the consideration of multi-scale uncertainty propagation, where the uncertainty can be modeled in the micromechanical properties in a physically more accurate way compared to the conventional macromechanical analyses, is scarce to find in the scientific literature. The present article aims to quantify the effect of spatially varying non-probabilistic uncertainty in composites following an efficient multi-scale RBF based framework. Thus a multi-scale approach is followed, wherein the effect of non-probabilistic uncertainty is included in the elementary micromechanical level first and then the effects are propagated towards the global responses (dynamics and stability) via an efficient surrogate of the actual finite element model. For this purpose, the concept of fuzzy representative volume element (FRVE) is proposed in the context of two-dimensional plate-like structures. This article hereafter is organized as, section 2: theoretical formulation for the fuzzy finite element analysis of composites; section 3: description of the RBF based fuzzy multi-scale 
uncertainty propagation algorithm; section 4: results and discussion demonstrating the influence of spatially varying fuzzy uncertainty on the dynamic and stability characteristics of composite laminates; section 5: summary and perspective of the present study in context to the available scientific literature; section 6: conclusion.

\section{Theoretical formulation for fuzzy finite element analysis of composites}

In the fuzzy concept of uncertainty, a set of transitional states across the members and nonmembers are described by a membership function $\left(\mu_{p_{i}}\right)$ that specifies the degree to which the elements in the domain belong to the fuzzy set [Zadeh (1975)]. Considering a triangular membership function, the fuzzy number can be given as,

$$
\tilde{p}_{i}\left(\tilde{\omega}_{\alpha}\right)=\left[p_{i}^{U}, p_{i}^{M}, p_{i}^{L}\right]
$$

Here $p_{i}^{M}, p_{i}^{U}$ and $p_{i}^{L}$ denote the mean, upper and lower bounds, respectively. The parameter $\tilde{\omega}_{\alpha}$ denotes the fuzziness corresponding to the $\alpha$-cut, in which $\alpha$ is known as the membership grade ranging from 0 to 1 .

The Gaussian probability distribution function can be approximated by a triangle for the fuzzy analysis [Hanss and Willner (2000)]. The area under triangular membership function is equated to the area under the normalised Gaussian distribution function to approximate the Gaussian probability distribution function as a triangle (refer to figure 2(a)) [Adhikari and Khodaparast (2014)], leading to a triangular fuzzy membership function as

$$
\mu_{p(i)}=\max \left[0,1-\frac{\left|X_{i}^{(j)}-\hat{X}_{i}\right|}{\zeta}\right]
$$

Here $\zeta=\sqrt{2 \pi \sigma_{X}}, \hat{X}_{i}$ and $\sigma_{X}$ represent the mean and standard deviation respectively, for the equivalent Gaussian distribution. The triangular shaped membership function $\left(\mu_{p(i)}\right)$, as employed in this work, can be described as 


$$
\begin{aligned}
& \mu_{p(i)}=1-\left(p_{i}^{M}-p_{i}\right) /\left(p_{i}^{M}-p_{i}^{L}\right), \quad \text { for } p_{i}^{L} \leq p_{i} \leq p_{i}^{M} \\
& \mu_{p(i)}=1-\left(p_{i}-p_{i}^{M}\right) /\left(p_{i}^{U}-p_{i}^{M}\right), \quad \text { for } p_{i}^{M} \leq p_{i} \leq p_{i}^{U} \\
& \mu_{p(i)}=0 \quad \text { Otherwise }
\end{aligned}
$$

Thus, the fuzzy input number $\left(p_{i}\right)$ can be defined into the set $P_{i}$ of $(m+1)$ intervals $p_{i}^{(j)}$ using the $\alpha$ cut method as below

$$
P_{i}\left(\tilde{\omega}_{\alpha}\right)=\left[p_{i}^{(0)}, p_{i}^{(1)}, p_{i}^{(2)} \ldots \ldots p_{i}^{(j)} \ldots \ldots p_{i}^{(m)}\right]
$$

Here $m$ is the number of $\alpha$-cuts. The interval of the $j$-th level of the $i$-th fuzzy number is expressed by

$$
p_{i}^{(j)}=\left[p_{i}^{(j, L)}, p_{i}^{(j, U)}\right]
$$

where $p_{i}^{(j, L)}$ and $p_{i}^{(j, U)}$ denote the lower and upper bounds of the interval (the superscripts $L$ and $U$ are used to denote the lower and upper bounds respectively) at the $j$-th level, where, $p_{i}^{(m, L)}=p_{i}^{(m, U)}=$ $p_{i}^{M}$ at $j=m$. A triangular fuzzy membership function as discussed in this section can represent a Gaussian distribution quite well. Besides that, it is logical to have equidistant bounds with respect to the mean values corresponding to different $\alpha$-cut levels when there is no experimental data available for the input parameters. However, the area equivalence analogy as presented here can be adopted for other distributions in future.

A numerical procedure of interval analysis at a number of $\alpha$-levels [Moens and Hanss (2011)] can be applied to propagate uncertainty in a system where the uncertain model parameters are constituted by fuzzy input numbers. In this analysis, the range of the response components (fuzzy output) on a specific level of membership function is searched within a particular $\alpha$-level in the input domain. Thus the analysis corresponding to each $\alpha$-cut resembles to an interval analysis for the system. Figure 2(b) [Moens and Vandepitte (2005, 2006); Adhikari and Khodaparast (2014)] depicts the scheme of fuzzy uncertainty propagation for a typical case of two input parameters and one output response with four $\alpha$-cut levels, but the idea can be readily generalised for the case of multiple input-output systems and any other number of $\alpha$-cut levels. In the approach adopted in this paper, we characterize a fuzzy variable with a set of interval variables by means of the membership function. The lower and upper bounds of interval variables at different $\alpha$-levels (i.e., $p_{i}^{(\alpha, L)}, p_{i}^{(\alpha, U)}$ ) 


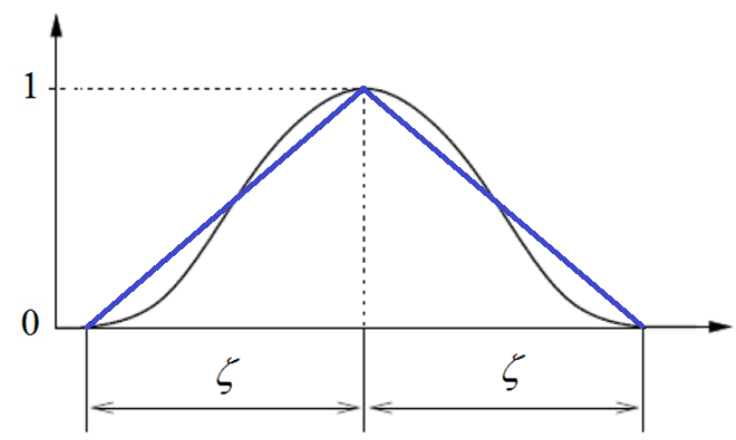

(a)

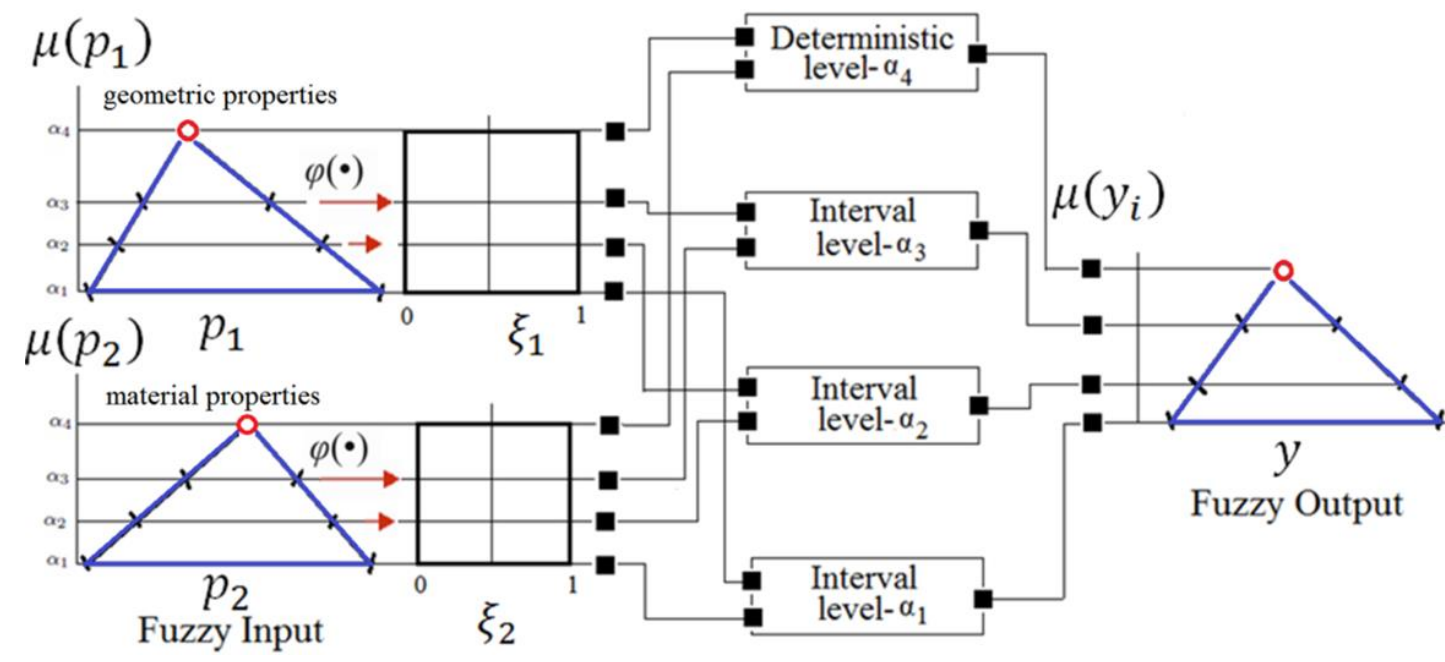

(b)

Fig. 2 (a) Linear approximation of a Gaussian distribution by triangular fuzzy membership functions (b) Scheme for fuzzy analysis containing transformation $\varphi(\cdot)$ of a fuzzy variable $p_{i}$ to $\xi \in[0,1]$ for different $\alpha$-cuts

can be converted into the normalized values in the range of 0 and 1 by means of a transformation function $\varphi(\bullet)$. The fuzzy uncertainty propagation begins with a deterministic solution at $\alpha=1$ and continues through an interval analysis towards the lower $\alpha$-cut levels. The interval variables ' $\xi_{i}$ ' are characterized by a normalised random variable with uniform probability distribution as

$$
p\left(\xi_{i}\right)=\left\{\begin{array}{ll}
\frac{1}{2} & 0<\xi_{i}<1 \\
0 & \text { elsewhere }
\end{array}\right\}
$$

If the input-output relationship of a particular system is known to be of monotonic nature, the lower and upper bounds of the fuzzy outputs corresponding to a particular $\alpha$-cut level can be found by carrying out very few number of simulations, otherwise a minimization and maximization algorithm needs to be employed involving multiple simulations. In case of such complex input- 
output relationships, an efficient surrogate based Monte Carlo simulation approach can be adopted to carry out the search for minimum and maximum values of the output quantities of interest for a particular $\alpha$-cut level. In this analysis, radial basis function [Beatson (1999), Fasshauer (1997), Hon and Mao (2001), Kansa (1990a, 1990b), Kansa and Hon (2000)] is employed as a surrogate [Mukhopadhyay et al. (2016b), Karsh et al. (2018b), Maharshi et al. (2018), Metya et al. (2017), Mahata et al. (2016), Dey et al. (2015, 2016d, 2016e, 2018b)] of the actual finite element model of composites.

The radial basis function model is dependent upon the distance to a central point $\hat{x}_{j}$ and a shape parameter called $c$ in some cases. For a set of nodes $\hat{x}_{1}, \hat{x}_{2}, \ldots \ldots \ldots . ., \hat{x}_{N} \in \Omega \subset R^{n}$, the $\mathrm{RBF}$ centred at $\hat{x}_{j}$ can be expressed as

$$
f_{j}(\hat{x}) \equiv f\left(\left\|\hat{x}-\hat{x}_{j}, c\right\|\right) \in R^{n}, j=1, \ldots \ldots, N
$$

where $\left\|\hat{x}-\hat{x}_{j}\right\|$ represents the Euclidian norm. The RBF models can be expressed as

$$
\begin{aligned}
& f_{j}(\hat{x}) \equiv e^{-c^{2}\left\|\hat{x}-\hat{x}_{j}\right\|^{2}} \\
& f_{j}(\hat{x}) \equiv\left(\left\|\hat{x}-\hat{x}_{j}\right\|+c^{2}\right)^{\frac{1}{2}} \quad \text { (Gaussian) } \\
& f_{j}(\hat{x}) \equiv\left(\left\|\hat{x}-\hat{x}_{j}\right\|^{2}+c^{2}\right)^{-\frac{1}{2}} \\
& f_{j}(\hat{x}) \equiv\left\|\hat{x}-\hat{x}_{j}\right\|^{2} \log \left\|\hat{x}-\hat{x}_{j}\right\| \\
& f_{j}(\hat{x}) \equiv\left\|\hat{x}-\hat{x}_{j}\right\|^{k}, k=1,3,5, \ldots \ldots . . \\
& f_{j}(\hat{x}) \equiv\left\|\hat{x}-\hat{x}_{j}\right\|^{k} \log \left\|\hat{x}-\hat{x}_{j}\right\|, k=2,4,6, \ldots . .
\end{aligned}
$$

RBFs are not sensitive to the spatial dimension making the implementation much easier for high dimensional systems [Beatson (1999), Powell (1987), Krishnamurthy (2003)]. RBF models do not need any grid; the only geometric property requirement is the pairwise distances between the points. In this paper, we have dealt with the spatially varying material properties for each lamina of the composite plate. Thus we need to deal with an exorbitantly high dimensional input parameter space 
depending on the number of FRVEs (refer to section 3). A surrogate modelling technique such as HDMR [Dey et al. (2016c), Mukhopadhyay et al. (2016a)] that requires inversion of a matrix to obtain the coefficients would be practically impossible to cope with the present problem. On the other hand, dealing with high dimensional input parameter spaces using RBF is not difficult as the distances are easy to evaluate in any number of space dimension. In this study, the RBF models are built using the quasi-random Sobol sequence, which has been reported to show a faster convergence than other sampling techniques [Mukhopadhyay et al. (2015), Mukhopadhyay (2018)]. In this context, it can be noted that the RBF models are required to be formed only for $\alpha=0$ in case of a fuzzy analysis. The same RBF models can be used for prediction in other $\alpha$-cut levels. Thus the number of expensive finite element simulations required for the present fuzzy analysis is same as the number of sample points drawn from Sobol sequence to form the RBF models corresponding to $\alpha=$ 0 level.

In the present analysis of laminated composites, the spatially varying material properties and ply orientation angles are considered as the source of fuzzy uncertainty, while the natural frequencies and buckling loads of the composite plate along with the respective mode shapes are the fuzzy output parameters of interest (denoted by $y$ in figure 2(b)). The governing equation for stochastic free vibration analysis without damping can be expressed as [Dey et al. (2016c)]

$$
\left[M\left(\tilde{\omega}_{\alpha}\right)\right]\left\{\ddot{\delta}\left(\tilde{\omega}_{\alpha}\right)\right\}+\left[K\left(\tilde{\omega}_{\alpha}\right)\right]\left\{\delta\left(\tilde{\omega}_{\alpha}\right)\right\}=0
$$

where $\left[K\left(\tilde{\omega}_{\alpha}\right)\right]=\left[K_{e}\left(\tilde{\omega}_{\alpha}\right)\right]+\left[K_{\sigma e}\left(\tilde{\omega}_{\alpha}\right)\right]$. Here $\left[K_{\sigma e}\left(\tilde{\omega}_{\alpha}\right)\right]$ and $\left[K_{e}\left(\tilde{\omega}_{\alpha}\right)\right]$ are the geometric stiffness matrix and elastic stiffness matrix respectively, while $\left[M\left(\tilde{\omega}_{\alpha}\right)\right]$ represents the mass matrix. In the finite element formulation of this study, an eight noded element is considered, wherein each node has five degrees of freedom (two rotations and three translations). The natural frequencies $\left(\omega_{k}\right)$ and mode shapes $\left(S_{k}^{f}\right)$ of the composite plate are obtained by solving an eigenvalue problem based on QR iteration algorithm (Bathe, 1990; Rayleigh, 1945)

$$
\left[K\left(\tilde{\omega}_{\alpha}\right)\right] S_{k}^{f}\left(\tilde{\omega}_{\alpha}\right)=\left[\omega_{k}\left(\tilde{\omega}_{\alpha}\right)\right]^{2}\left[M\left(\tilde{\omega}_{\alpha}\right)\right] S_{k}^{f}\left(\tilde{\omega}_{\alpha}\right)
$$


where $k=1,2,3, \ldots ., n$. The superscript $f$ is used to denote frequency analysis. Here the orthogonality relationship is satisfied as

$$
\left[S_{i}^{f}\left(\tilde{\omega}_{\alpha}\right)\right]^{T}\left[M\left(\tilde{\omega}_{\alpha}\right)\right] S_{k}^{f}\left(\tilde{\omega}_{\alpha}\right)=\bar{\delta}_{i k} \text { and }\left[S_{i}^{f}\left(\tilde{\omega}_{\alpha}\right)\right]^{T}\left[K\left(\tilde{\omega}_{\alpha}\right)\right] S_{k}^{f}\left(\tilde{\omega}_{\alpha}\right)=\left[\omega_{k}\left(\tilde{\omega}_{\alpha}\right)\right]^{2} \bar{\delta}_{i k}
$$

where $i, k=1,2,3, \ldots . n$ and the Kroneker delta functions $\bar{\delta}_{i k}=0$ for $i \neq k ; \bar{\delta}_{i k}=1$ for $i=k$. It can be noted here that we have followed a Monte Carlo simulation based approach for the present fuzzy analysis of composite structures. A Monte Carlo simulation involves multiple realizations of the finite element model considering the input parameters drawn algorithmically from a certain random sequence. Thus each of the realizations of a Monte Carlo simulation can be regarded as a separate deterministic analysis in the present non-intrusive approach. This essentially means that even though the current investigation is collectively a stochastic analysis, the orthogonality relationship is satisfied.

The problem of stability analysis is solved through another eigenvalue problem obtained by minimizing the total potential energy as:

$$
\left[K_{e}\left(\tilde{\omega}_{\alpha}\right)\right] S_{k}^{b}=\lambda^{b}\left(\tilde{\omega}_{\alpha}\right)\left[K_{\sigma e}\left(\tilde{\omega}_{\alpha}\right)\right] S_{k}^{b}\left(\tilde{\omega}_{\alpha}\right)
$$

where $\lambda^{b}\left(\tilde{\omega}_{\alpha}\right)$ is the fuzzy buckling load factor and $S_{k}^{b}$ gives the buckling modeshapes. The superscript $b$ is used to denote frequency analysis. The detail fuzzy finite element formulation for composites is provided as a supplementary material.

\section{Fuzzy representative volume element based framework for non-probabilistic uncertainty quantification}

\subsection{Concept of FRVE}

In this paper a concept of fuzzy representative volume element (FRVE) is proposed for twodimensional plate-like structures to account for the effect of spatial variation of material properties. According to this approach, each of the representative units (structural element) is considered to be stochastic in nature with different material properties, instead of considering the homogenized mechanical properties of a conventional representative volume element (RVE) throughout the entire 


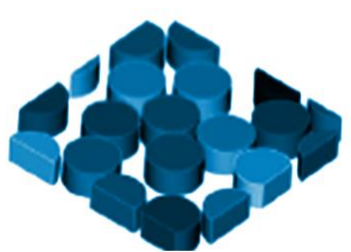

Fibres

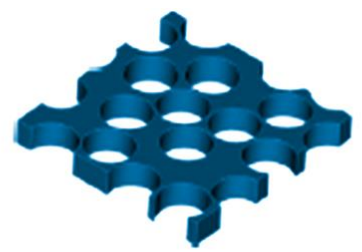

Matrix

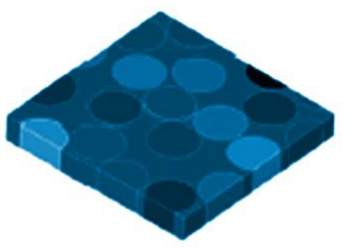

Full RVE

Fig. 3 Conventional RVE for unidirectional fiber reinforced composites

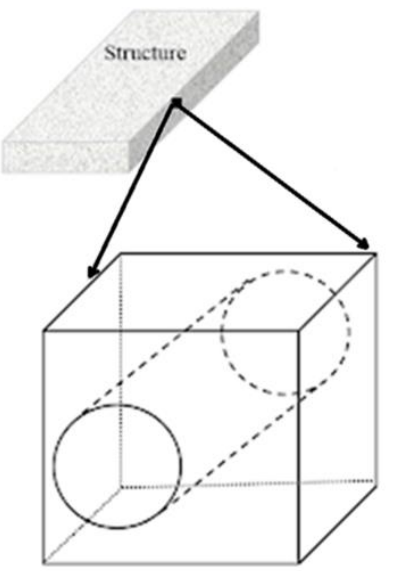

FRVE

(a)

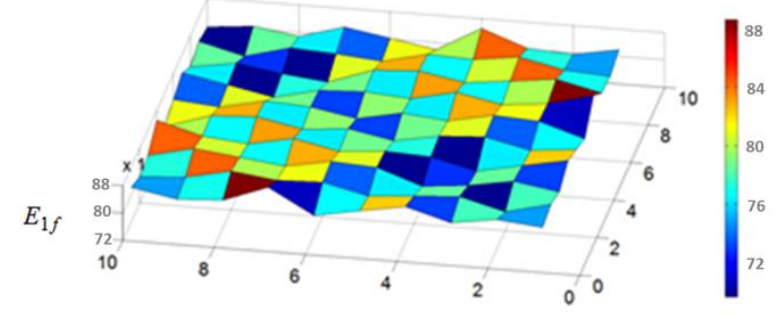

(c)

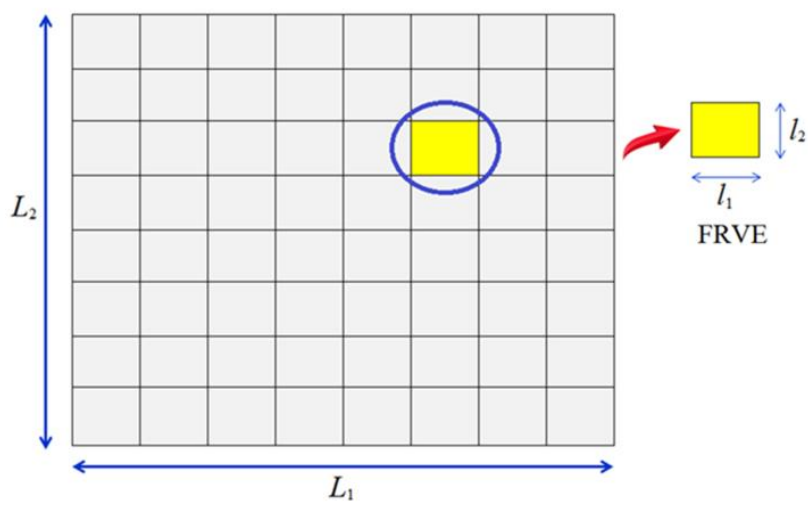

(b)

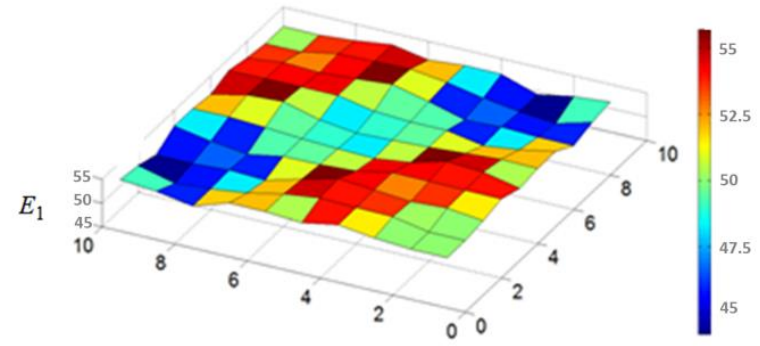

(d)

Fig. 4 (a -b) FRVE based approach for analyzing spatially varying fuzzy uncertainty (c - d) Typical illustrative representation of spatially varying material properties for longitudinal Young's modulus of fiber (a micromechanical property) and Young's modulus (in GPa) of the composite at macroscale

solid domain (refer to figure 3). As per the traditional approach, one RVE is analysed typically and the assumption is that a single RVE can represent the entire analysis domain [Sriramula and Chryssanthopoulos (2009)]. However, this approach of analysis can lead to eroneous outcomes, specially in case of stochastic systems with spatial variability in material and other attributes. To analyse such systems, it is essential to account for the effect of the randomness of stocahstic mechanical properties along the spatial location of different zones of a plate-like structure. 
According to the present approach, the entire plate is assumed to be consisted of a finite number of FRVEs. Thus mechanical properties of a FRVE are dependent on its stochastic material and structural properties and they are different for each of the FRVEs in a particular realization of Monte Carlo simulation. In this study, it is considered that all the FRVEs have same membership function for a particular material property. Following this framework, it becomes feasible to consider the spatial randomness (/ non-probabilistic uncertainty) in a structural system more realistically. The global responses (such as natural frequencies and buckling loads) of the plate are computed by propagating the mechanical information acquired in the elementary local level (FRVEs) towards the global level by combining (/assembling) the FRVEs applying the principles of solid mechanics (finite element approach in the present study). Recently, a similar concept has been proposed for analyzing hexagonal honeycomb-like lattices having probabilistic spatial irregularity [Mukhopadhyay and Adhikari (2016a, 2017a), Mukhopadhyay et al. (2017, 2018a, 2018b), Mahata and Mukhopadhyay (2018)], wherein multiple representative unit cell elements (RUCE) are analysed instead of the conventional approach of considering a single unit cell. The entire lattice structure is assumed to be consisted of several RUCEs and the global mechanical properties of the entire irregular lattice can be computed by assembling the RUCEs based on equilibrium and compatibility conditions. A concept of SRVE (stochastic representative volume element) for probabilistic analysis with random material properties and crack density is adopted by Naskar et al. (2017a, 2018). In this paper, we have developed the FRVE based concept for non-probabilistic analysis of two-dimensional plate-like structures with inhomogeneous [Mukhopadhyay and Adhikari (2017b)] form of fuzzy uncertainty. The adoption of FRVEs in a plate-like structure is shown in the figure 4, wherein the entire domain is divided into a finite number of fuzzy elements (FRVEs) having dimensions of $l_{1}$ and $l_{2}$ in two mutually perpendicular directions. Here, a parameter characteristic length $(r)$ can be defined as: $r=\frac{l_{1}}{L_{1}}=\frac{l_{2}}{L_{2}}=\frac{1}{N_{d}}$, where $N_{d}$ denotes the number of divisions along the two dimensions of the plate. It can be noted that when $r \geq 1$, the fuzzy system would behave like the conventional form of nonprobabilistic analysis without considering any spatial variation, as presented by Dey et al. (2016b). 
As per the proposed concept of FRVE, the size (/number) of FRVE is independent of the discretization in a finite element based numerical solution that could be adopted for dynamic/ stability analysis of the composite plate. Once the size of a FRVE is decided, they could be discretized following conventional finite element analysis using a mesh convergence study. In the present analysis, we have only considered spatial variation of micro and macro scale material properties; but future studies could include random spatial variation of microstructural properties (such as micro-scale damage) using the proposed FRVE based framework. In such problems, appropriate finite element meshing schemes would need to be adopted for each of the FRVEs.

\subsection{RBF based FRVE approach for spatially varying fuzzy uncertainty propagation}

The stochasticity in material properties (micro/ macro- mechanical properties) and geometric properties (like ply-orientation angle and thickness of plate) are considered as fuzzy input parameters for analyzing the dynamic and stability characteristics of laminated composite plates. In the present article, two separate forms of analysis have been performed considering the stochasticity in micromechanical and macro-mechanical properties to understand and ascertain the relative effect in nonprobabilistic uncertainty propagation on a comparative basis. For analysing the effect of various source-uncertainties, the following five cases are considered:

(i) Compound effect for the simultaneous variation of macro-mechanical material properties (such as Young's moduli, shear moduli, mass density and Poisson's ratio) and geometric properties (such as ply-orientation angle and thickness of laminae)

$$
\begin{aligned}
g_{\text {macro }}^{C}\{ & \left.E_{1}\left(\tilde{\omega}_{\alpha}\right), E_{2}\left(\tilde{\omega}_{\alpha}\right), G_{12}\left(\tilde{\omega}_{\alpha}\right), G_{13}\left(\tilde{\omega}_{\alpha}\right), G_{23}\left(\tilde{\omega}_{\alpha}\right), \mu\left(\tilde{\omega}_{\alpha}\right), \rho\left(\tilde{\omega}_{\alpha}\right), \theta\left(\tilde{\omega}_{\alpha}\right), t\left(\tilde{\omega}_{\alpha}\right)\right\} \\
= & \left\{\begin{array}{l}
\phi_{\text {macro }}^{1}\left(E_{1(1,1)} \ldots . E_{1(e, l)}\right), \phi_{\text {macro }}^{2}\left(E_{2(1,1)} \ldots E_{2(e, l)}\right), \phi_{\text {macro }}^{3}\left(G_{12(1,1)} \ldots G_{12(e, l)}\right), \\
\phi_{\text {macro }}^{4}\left(G_{13(1,1)} \ldots G_{13(e, l)}\right), \phi_{\text {macro }}^{5}\left(G_{23(1,1)} \ldots . G_{23(e, l)}\right), \phi_{\text {macro }}^{6}\left(\mu_{(1,1)} \ldots . \mu_{(e, l)}\right), \\
\phi_{\text {macro }}^{7}\left(\rho_{(1,1)} \ldots . \rho_{(e, l)}\right), \phi_{\text {macro }}^{8}\left(\theta_{(1,1)} \ldots . \theta_{(e, l)}\right), \phi_{\text {macro }}^{9}\left(t_{(1,1)} \ldots . t_{(e, l)}\right)
\end{array}\right\}
\end{aligned}
$$

(ii) Compound effect for the simultaneous variation of micro-mechanical material properties such as Young's moduli of fibre and matrix, shear moduli of fibre and matrix, Poisson ratios of fibre and matrix, mass densities of fibre and matrix and volume fraction along with geometric properties (ply orientation angle and thickness of laminae) 


$$
\begin{aligned}
g_{\text {micro }}^{C} & \left\{E_{1 f}\left(\tilde{\omega}_{\alpha}\right), E_{2 f}\left(\tilde{\omega}_{\alpha}\right), E_{m}\left(\tilde{\omega}_{\alpha}\right), G_{f}\left(\tilde{\omega}_{\alpha}\right), G_{m}\left(\tilde{\omega}_{\alpha}\right), \mu_{f}\left(\tilde{\omega}_{\alpha}\right), \mu_{m}\left(\tilde{\omega}_{\alpha}\right), \rho_{f}\left(\tilde{\omega}_{\alpha}\right), \rho_{m}\left(\tilde{\omega}_{\alpha}\right), V_{f}\left(\tilde{\omega}_{\alpha}\right), \theta\left(\tilde{\omega}_{\alpha}\right), t\left(\tilde{\omega}_{\alpha}\right)\right\} \\
= & \left\{\begin{array}{l}
\phi_{\text {micro }}^{1}\left(E_{1 f(1,1)} \ldots . E_{1 f(e, l)}\right), \phi_{\text {micro }}^{2}\left(E_{2 f(1,1)} \ldots E_{2 f(e, l)}\right), \phi_{\text {micro }}^{3}\left(E_{m(1,1)} \ldots E_{m(e, l)}\right), \phi_{\text {micro }}^{4}\left(G_{f(1,1)} \ldots G_{f(e, l)}\right), \\
\phi_{\text {micro }}^{5}\left(G_{m(1,1)} \ldots . G_{m(e, l)}\right), \phi_{\text {micro }}^{6}\left(\mu_{f(1,1)} \ldots ., \mu_{f(e, l)}\right), \phi_{\text {micro }}^{7}\left(\mu_{m(1,1)} \ldots, \mu_{m(e, l)}\right), \phi_{\text {micro }}^{8}\left(\rho_{f(1,1)} \ldots \rho_{f(e, l)}\right), \\
\phi_{\text {micro }}^{9}\left(\rho_{m(1,1)} \ldots \rho_{m(e, l)}\right), \phi_{\text {micro }}^{10}\left(V_{f(1,1)} \ldots . V_{f(e, l)}\right), \phi_{\text {micro }}^{11}\left(\theta_{f(1,1)} \ldots \theta_{f(e, l)}\right), \phi_{\text {micro }}^{12}\left(t_{f(1,1)} \ldots . t_{f(e, l)}\right)
\end{array}\right\}
\end{aligned}
$$

(iii) Individual effect for the variation of a single macro-mechanical property

$$
g_{\text {macro }}^{I}\left\{\kappa_{M}\left(\tilde{\omega}_{\alpha}\right)\right\}=\left\{\phi_{\text {macro }}^{1}\left(\kappa_{M(1,1)} \ldots . \kappa_{M(e, l)}\right)\right\}
$$

(iv) Individual effect for the variation of a single micro-mechanical property

$$
g_{\text {micro }}^{I}\left\{\kappa_{m}\left(\tilde{\omega}_{\alpha}\right)\right\}=\left\{\phi_{\text {micro }}^{1}\left(\kappa_{m(1,1)} \ldots . \kappa_{m(e, l)}\right)\right\}
$$

(v) Individual effect for the variation of a single geometric property

$$
g_{G}^{I}\left\{\kappa_{G}\left(\tilde{\omega}_{\alpha}\right)\right\}=\left\{\phi_{G}^{1}\left(\kappa_{G(1,1)} \ldots \kappa_{G(e, l)}\right)\right\}
$$

where, $E_{1(i, j)}, E_{2(i, j)}, G_{12(i, j)}, G_{13(i, j)}, G_{23(i, j)}, \mu_{(i, j)}, \rho_{(i, j)}, \theta_{(i, j)}, t_{(i, j)}$ denote the longitudinal Young's modulus, transverse Young's modulus, shear moduli, Possoin's ratio, mass density, ply orientation angle and thickness of lamina respectively (with conventional notations) for the $i^{\text {th }}$ FRVE situated in the $j^{\text {th }}$ layer, where $i=1,2,3, \ldots, e$ and $j=1,2,3, \ldots, l$. The function $g$ in the above expressions is used to denote a particular type of stochastic analysis, as evident from the involved elements in the expressions. The parameter $\phi$ in the expressions is a symbolic representation of Monte Carlo simulation. For the stochasticity in micro-mechanical properties, $E_{1 f(i, j)}, E_{2 f(i, j)}, E_{m(i, j)}, G_{f(i, j)}, G_{m(i, j)}, \mu_{f(i, j)}, \mu_{m(i, j)}, \rho_{f(i, j)}, \rho_{m(i, j)}, V_{f(i, j)}, \theta_{(i, j)}, t_{(i, j)}$ denote (following conventional notations) Young's moduli of fibre in longitudinal and transverse directions, Young's modulus of matrix, shear modulus of fibre and matrix, Poisson's ratio of fibre and matrix, mass density of fibre and matrix and volume fraction, ply orientation angle and thickness respectively corresponding to $i^{\text {th }}$ layer, respectively for the $i^{\text {th }}$ FRVE situated in the $j^{\text {th }}$ layer, where $i=1,2,3, \ldots, e$ and $j=1,2,3, \ldots, l$. The quantities $\kappa_{M}$ and $\kappa_{m}$ denote any one of the macromechanical and micromechanical properties, while $\kappa_{G}$ is any one of the geometric properties. The material properties are considered to vary spatially for both the macromechanical and micromechanical analyses. 


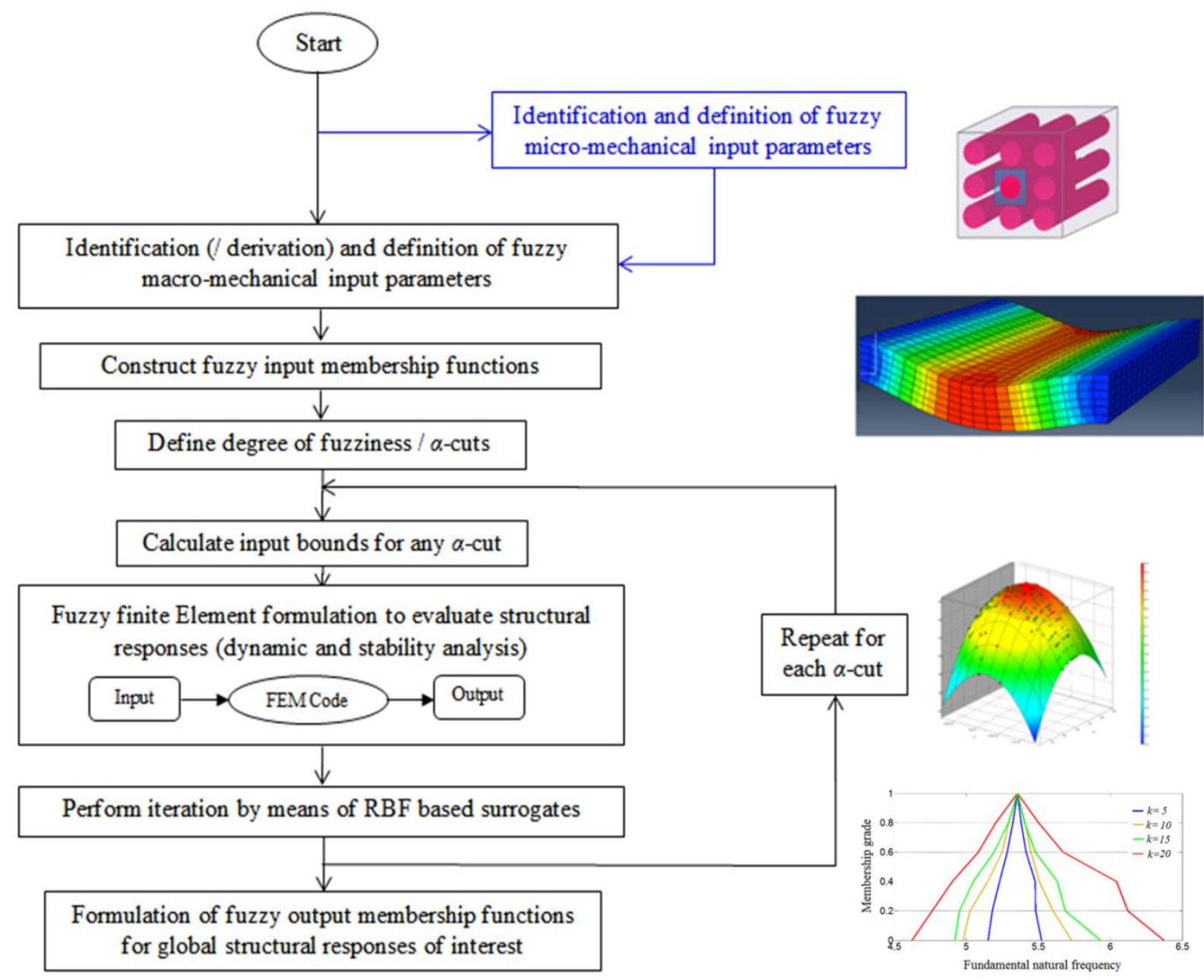

Fig. 5 Flowchart for RBF based fuzzy multi-scale analysis of composites (Representative figures of multi-scale finite element analysis, surrogate modelling and fuzzy uncertainty quantification are shown)

However, considering practical aspects of modelling uncertainty in the geometric parameters from the manufacturing point of view, spatial variation is neglected for ply orientation angle and thickness of lamina; rather a layer-wise variation is considered for the two geometric parameters (i.e. $\theta_{(1, j)}=\theta_{(2, j)}=\theta_{(3, j)}=\ldots=\theta_{(e, j)}$ and $t_{(1, j)}=t_{(2, j)}=t_{(3, j)}=\ldots=t_{(e, j)}$, for the $j^{\text {th }}$ lamina). Thus, we have adopted a mixed form of uncertainty modelling with spatial variability in the material properties and random fibre orientation angle with no relative variation in the direction along the profile of the fibres. For example, if the deterministic value of a certain ply angle is $45^{\circ}$, one particular stochastic realization could assume the value of $45.1^{\circ}$ throughout the fiber in a straight profile.

In a typical problem of uncertainty analysis, there are normally three aspects that need to be dealt with. The first aspect is source-uncertainty modelling at the input level, for which a realistic spatially varying non-probabilistic modelling approach is adopted in this article. After the uncertainty 
in material and structural attributes is modelled in a practically relevant way, the next concern is propagating the effect of uncertainty from the local input-level to the global level for quantifying output responses following a bottom-up framework. As the input-output relationships of composites are normally complicated (/non-monotonic) in nature, a Monte Carlo simulation based approach can be adopted for finding the response bounds corresponding to each $\alpha$-cuts. However, direct Monte Carlo simulation involving finite element models being a computationally intensive approach, we have adopted a surrogate based scheme (RBF) in conjunction with the fuzzy finite element model of composite plates as presented in figure 5 .

\section{Results and Discussion}

In this article, numerical results for fuzzy dynamic and stability analyses are presented for a three layered graphite-epoxy cross-ply $\left(\left[0^{\circ} / 90^{\circ} / 0^{\circ}\right]\right)$ composite plate (clamped at the four edges) with an aspect ratio of 2 and degree of fuzziness $k=10$, unless otherwise mentioned. It can be noted here that the degree of fuzziness $(k)$ indicates the bound of input parameters with respect to the respective nominal value at $\alpha=0$ (i.e. $\pm k \%$ ). A practically relevant spatially varying non-probabilistic approach of uncertainty modelling is considered for characterizing the first three modes of vibration and buckling of composite plates. Results are presented for two distinct cases: fuzzy uncertainty in micromechanical and macromechanical material properties (refer to equation $13-17$ ). The deterministic micromechanical properties (E-glass 21xK43Gevetex/ 3501-6 epoxy) of composite material are shown in Table 1 [Soden et al. (1998)]. Applying Halpin-Tsai principle [Jones (1999)] the deterministic macromechanical material properties are obtained with a volume fraction $\left(V_{f}\right)$ of 0.61 (refer to Table 2). Thus, for the case of stochasticity in micromechanical properties, the material attributes presented in Table 1 are assumed as the source of fuzzy uncertainty along with the uncertain geometric parameters $\left(g_{\text {micro }}^{C}\right)$ and thereby the macromechanical material properties are obtained based on Halpin - Tsai principle to perform further analysis for quantifying uncertainty. For the case of fuzzy uncertainty in macromechanical material properties, the analysis commences one step ahead in the hierarchy i.e. the fuzzy source-uncertainty is assumed in the macromechanical 
Table 1 Micromechanical material properties (deterministic) of composites

\begin{tabular}{|c|c|}
\hline Material property & Numerical value \\
\hline Longitudinal Young's modulus of fibre $\left(E_{1 f}\right)$ & $80 \times 10^{3}$ (Unit: $\left.\mathrm{MPa}\right)$ \\
\hline Transverse Young's modulus of fibre $\left(E_{2 f}\right)$ & $80 \times 10^{3}$ (Unit: $\left.\mathrm{MPa}\right)$ \\
\hline Poisson's ratio of fibre $\left(\mu_{f}\right)$ & 0.2 \\
\hline Shear modulus of matrix $\left(G_{f}\right)$ & $33.33 \times 10^{3}$ (Unit: MPa) \\
\hline Mass density of fibre $\left(\rho_{f}\right)$ & 2.55 (Unit: gm/cc) \\
\hline Mass density of matrix $\left(\rho_{m}\right)$ & 1.26 (Unit: gm/cc) \\
\hline Young's modulus of matrix $\left(E_{m}\right)$ & $4.2 \times 10^{3}$ (Unit: $\left.\mathrm{MPa}\right)$ \\
\hline Shear modulus of matrix $\left(G_{m}\right)$ & $1.567 \times 10^{3}$ (Unit: MPa) \\
\hline Poisson's ratio of matrix $\left(\mu_{m}\right)$ & 0.34 \\
\hline Fibre volume fraction $\left(V_{f}\right)$ & 0.61 \\
\hline
\end{tabular}

Table 2 Macromechanical material properties (deterministic) of composites $\left(V_{f}=0.61\right)$

\begin{tabular}{c|c}
\hline Material property & Numerical value \\
\hline Longitudinal Young's modulus $\left(E_{1}\right)$ & $50.438 \times 10^{3}($ Unit: MPa) \\
Transverse Young's modulus $\left(E_{2}\right)$ & $9.952 \times 10^{3}($ Unit: MPa $)$ \\
Poisson's ratio $\left(\mu_{12}\right)$ & 0.2546 \\
In-plane shear modulus $\left(G_{12}\right)$ & $3.742 \times 10^{3}($ Unit: MPa) \\
Mass density $(\rho)$ & $2.049 \times 10^{3}($ Unit: gm/cc) \\
Shear modulus $\left(G_{13}\right)$ & $3.742 \times 10^{3}($ Unit: MPa) \\
Transverse shear modulus $\left(G_{23}\right)$ & $2.094 \times 10^{3}($ Unit: MPa) \\
\hline
\end{tabular}




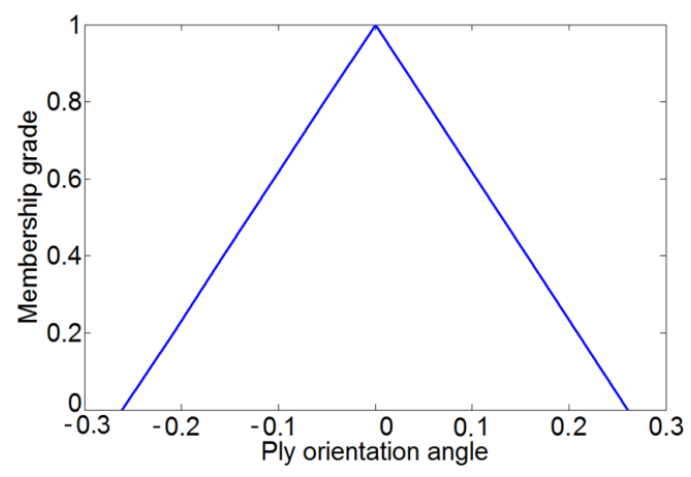

(a)

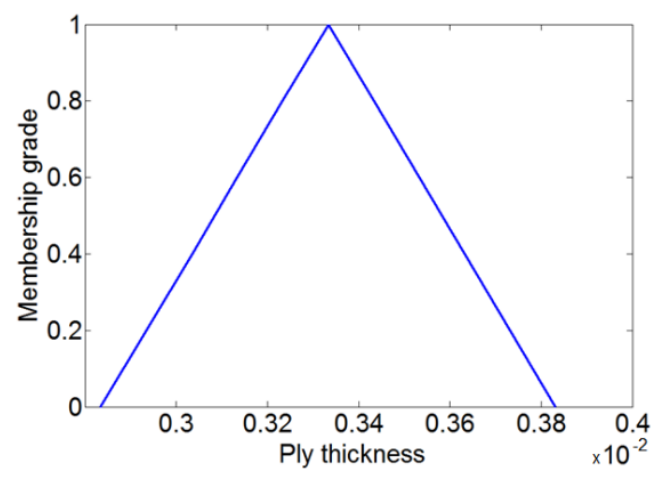

(b)

Fig. 6 Typical representation of membership functions for the geometric parameters ply orientation angle in rad (with a nominal value of $0 \mathrm{rad}$ ) and thickness in $\mathrm{m}$ (with a nominal value of $0.33 \times 10^{-2} \mathrm{~m}$ )

properties (as shown in Table 2) along with uncertain geometric parameters $\left(g_{\text {macro }}^{C}\right)$. Subsequently, the results obtained from these two different types of non-probabilistic analyses are compared to ascertain the relative effect. Typical plots for the membership functions considered in the geometric properties are shown in figure 6. Figure 7(a) and 7(b) show the representative membership functions for micromechanical properties and macromechanical properties in a micromechanical analysis, while figure $7(\mathrm{c})$ shows the membership functions for macromechanical properties in a macromechanical analysis. It can be noted that figure 7(b) is obtained from figure 7(a) based on the Halpin - Tsai principle following a fuzzy interpretation. In the present study, $\pm k^{\circ}$ variation for ply orientation angle and $\pm k \%$ variation for material properties from fuzzy crisp values are considered corresponding to $\alpha=0$, where $k$ is referred as the degree of fuzziness. Non-dimensional results for natural frequencies are presented as $\bar{\omega}=\left(\omega b^{2} / \pi^{2}\right) \sqrt{\left(\rho h / D_{0}\right)}$, where $D_{0}=E_{2} h^{3} / 12\left(1-v_{12} v_{21}\right)$, while for buckling load the adopted non-dimensionalisation scheme is: $\bar{N}=\bar{N}_{x x} b^{2} /\left(E_{2} h^{3}\right), \bar{N}_{x y}=0, \bar{N}_{y y}=0$.

\subsection{Fuzzy dynamic analysis}

\subsubsection{Validation and convergence study}

In the RBF based fuzzy analysis of laminated composites, two different forms of validation and convergence study are needed to be carried out. The first validation is for the finite element model of the composite plate (in-house FE code written in MATLAB) along with mesh convergence 


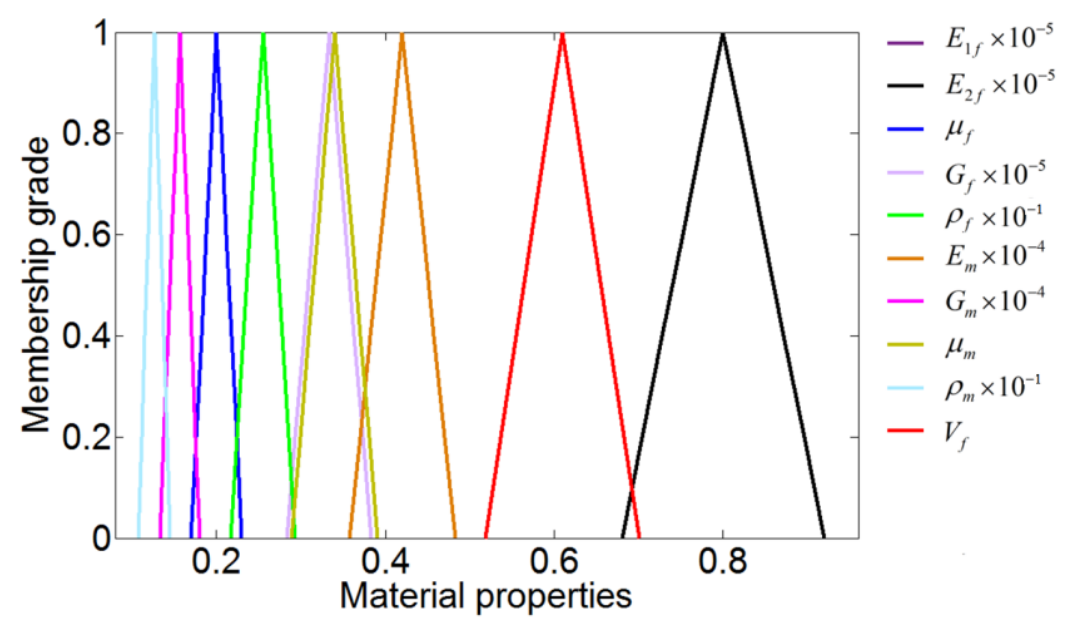

(a)

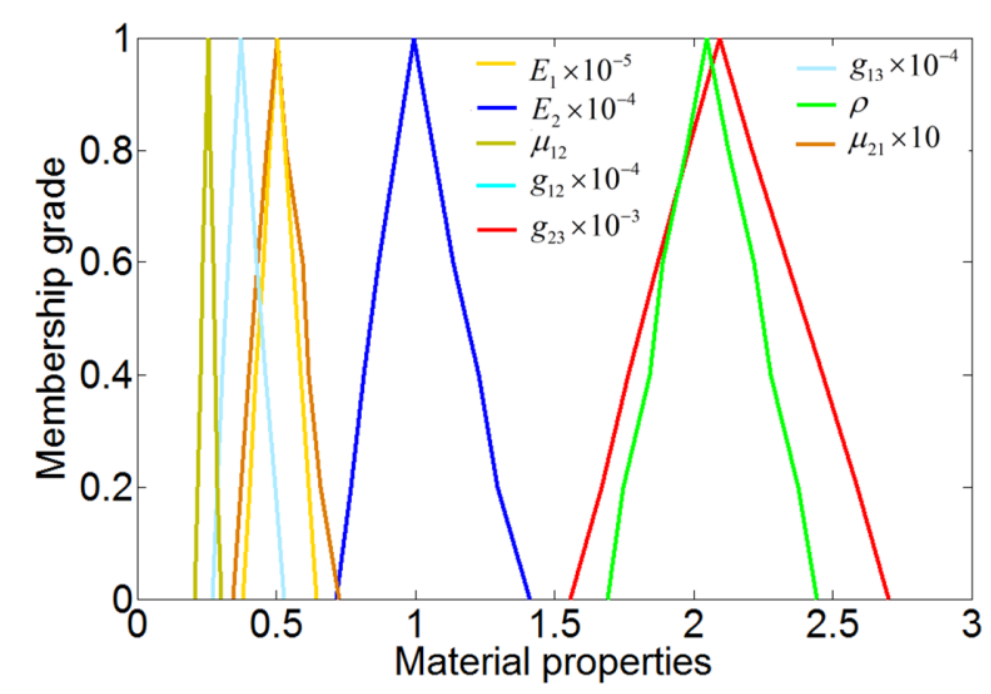

(b)

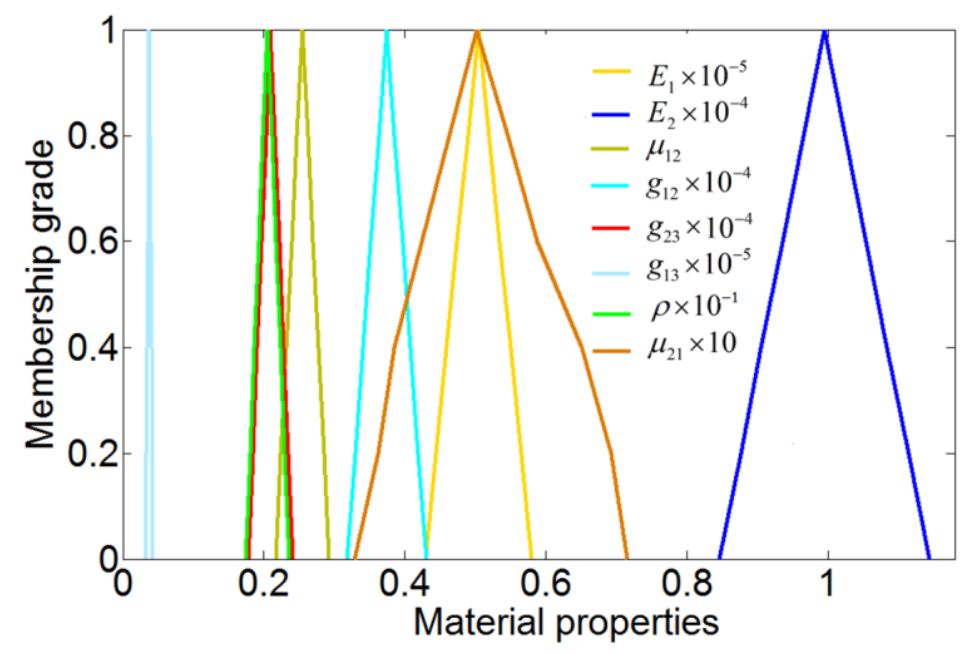

(c)

Fig. 7 (a) Typical representation of membership functions for the micromechanical properties in micromechanical analysis (b) Typical representation of membership functions for the macromechanical properties in micromechanical analysis (c) Typical representation of membership functions for the macromechanical properties in macromechanical analysis (The units are in accordance with the nominal values presented in Table 1 and 2) 


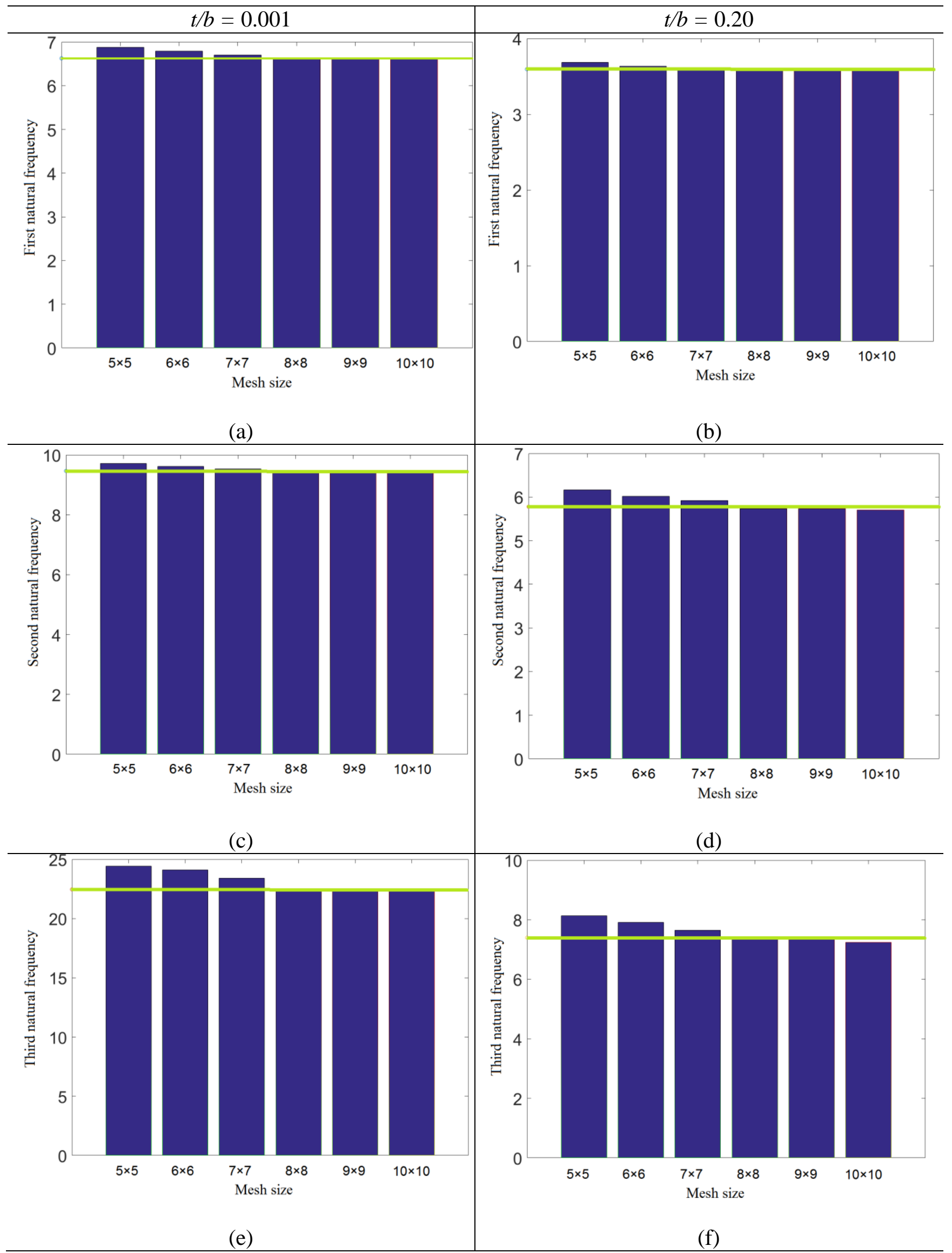

Fig. 8 The convergence study of frequency parameters for cross-ply $\left(0^{\circ} / 90^{\circ} / 0^{\circ}\right)$ simply supported (SSSS) rectangular laminates. The reference results from Liew (1996) are shown by the green lines. The results are shown for two different $t / b$ ratios. 


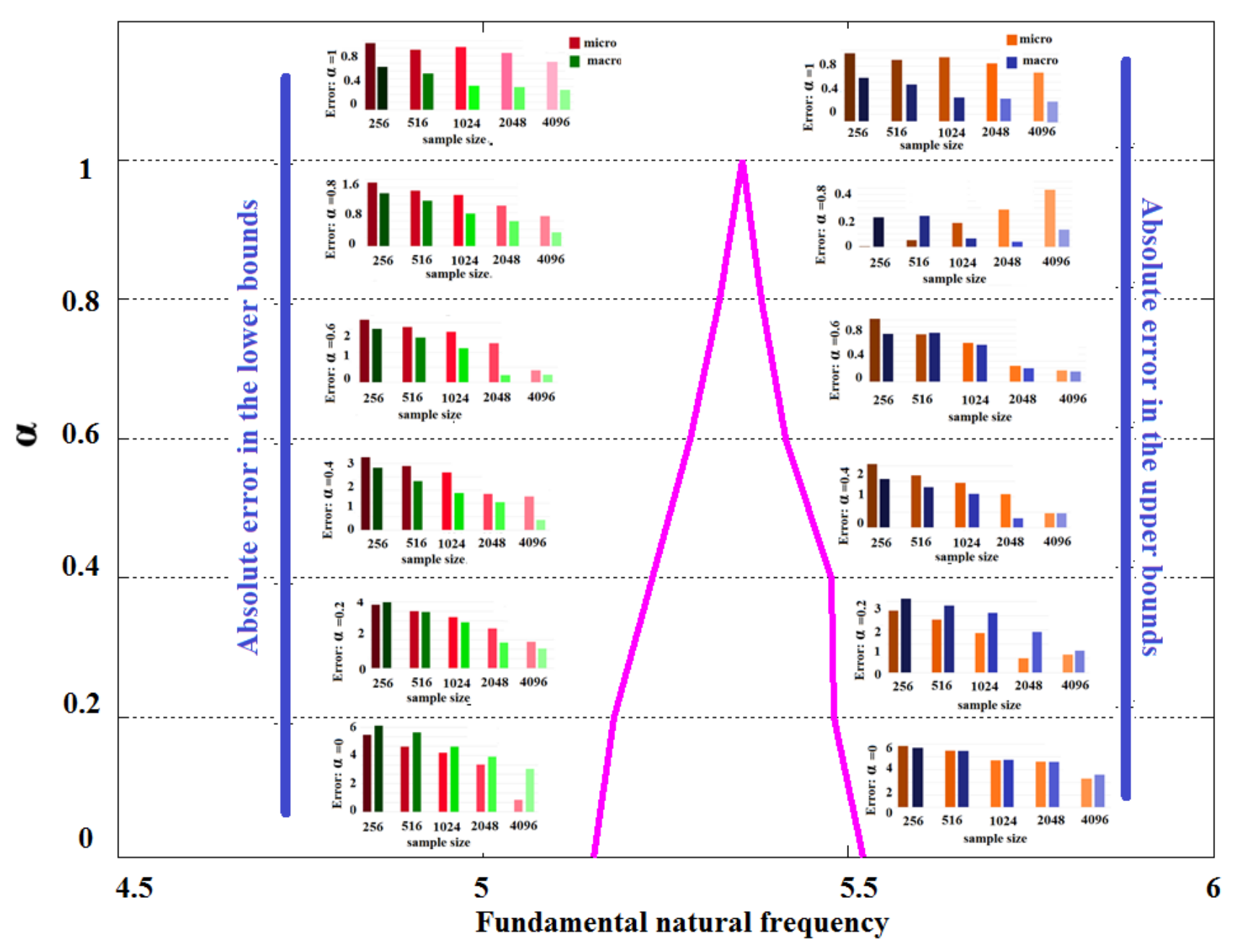

Fig. 9 Convergence study and validation for the sample size required to form the RBF model in case of first natural frequency $\left(g_{\text {micro }}^{C}\right.$ and $g_{\text {macro }}^{C}$ ). A typical membership function is shown considering the compound effect of stochasticity in micromechanical parameters $\left(g_{\text {micro }}^{C}\right)$.

study. A second type of validation is also needed here concerning the performance (efficiency and accuracy) of the RBF model in predicting the responses along with a convergence study for minimizing the number of design points (i.e. number of expensive finite element simulations) required for forming the surrogate models.

The results for validation and convergence study of the deterministic finite element code of a composite plate are shown in figure 8, wherein non-dimensional natural frequencies are validated with the results available in scientific literature [Liew and Huang (2003)]. Based on the results presented in figure 8 , a mesh size of $9 \times 9$ is adopted for the finite element analysis hereafter. The optimum number of samples (drawn from Sobol sequence) to construct the RBF model are decided based on the comparative performance (absolute error in predicting the lower and upper bounds for 


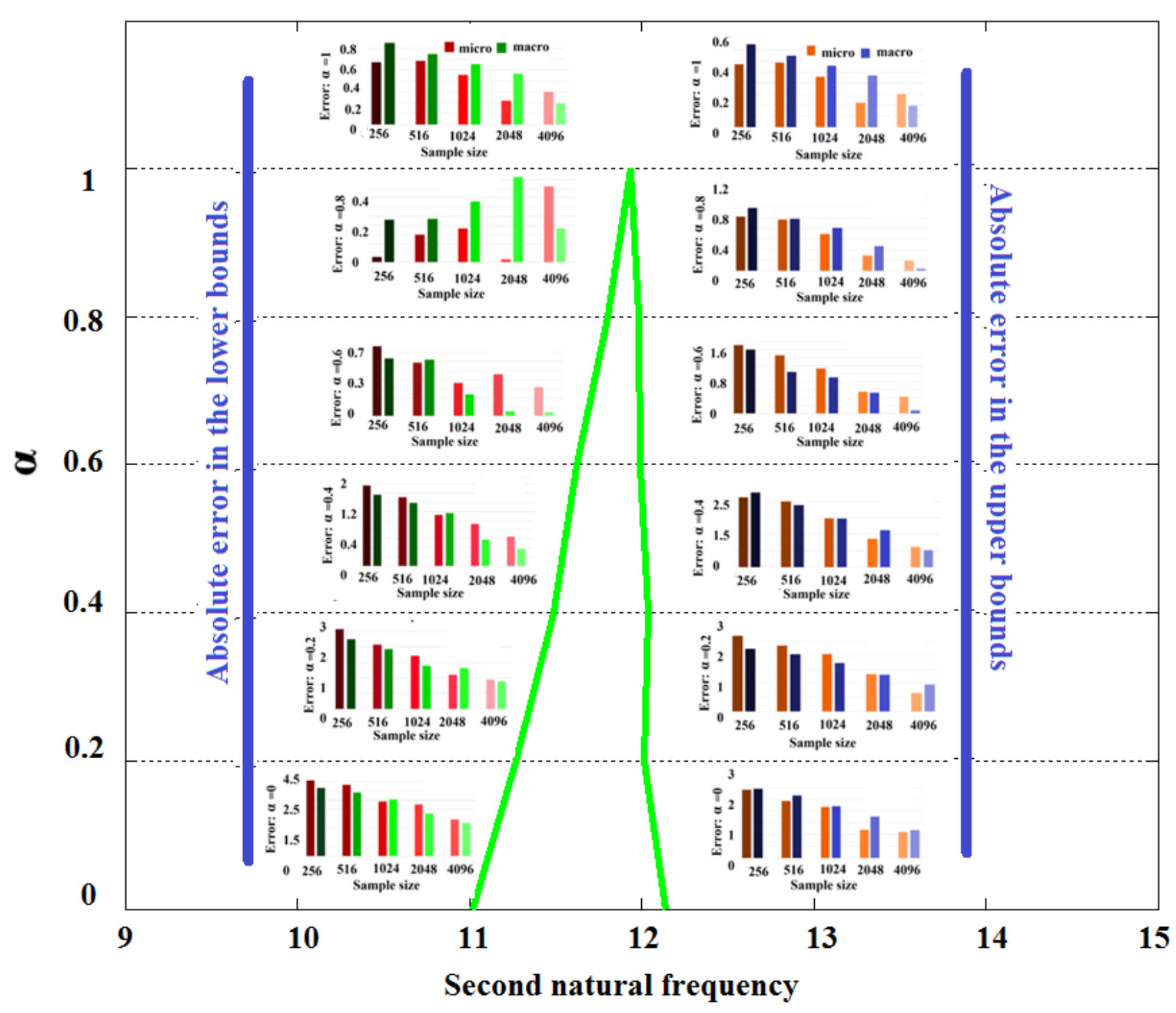

Fig. 10 Convergence study and validation for the sample size required to form the RBF model in case of second natural frequency $\left(g_{\text {micro }}^{C}\right.$ and $g_{\text {macro }}^{C}$ ). A typical membership function is shown considering the compound effect of stochasticity in micromechanical parameters $\left(g_{\text {micro }}^{C}\right)$.

each of the $\alpha$-cuts) with respect to the direct Monte Carlo simulation approach. The results for macro and micro mechanical analyses showing the values of absolute error for different sample sizes corresponding to different $\alpha$-cuts are presented in figure $9-11$. From the figures it is evident that a sample size of 4096 provides reasonably accurate results for the natural frequencies. It can be noted in this context that the RBF model is formed only for $\alpha=0$, while the same RBF model can be used for prediction corresponding to the other $\alpha$-cuts. The lower and upper bounds are found based on a Monte Carlo simulation based approach that requires a large number of finite element simulations $\left(\sim 10^{4}\right)$ for each of the $\alpha$-cuts. Hence, the computational time and cost in terms of finite element analyses are reduced significantly compared to the direct Monte Carlo simulation based approach of 


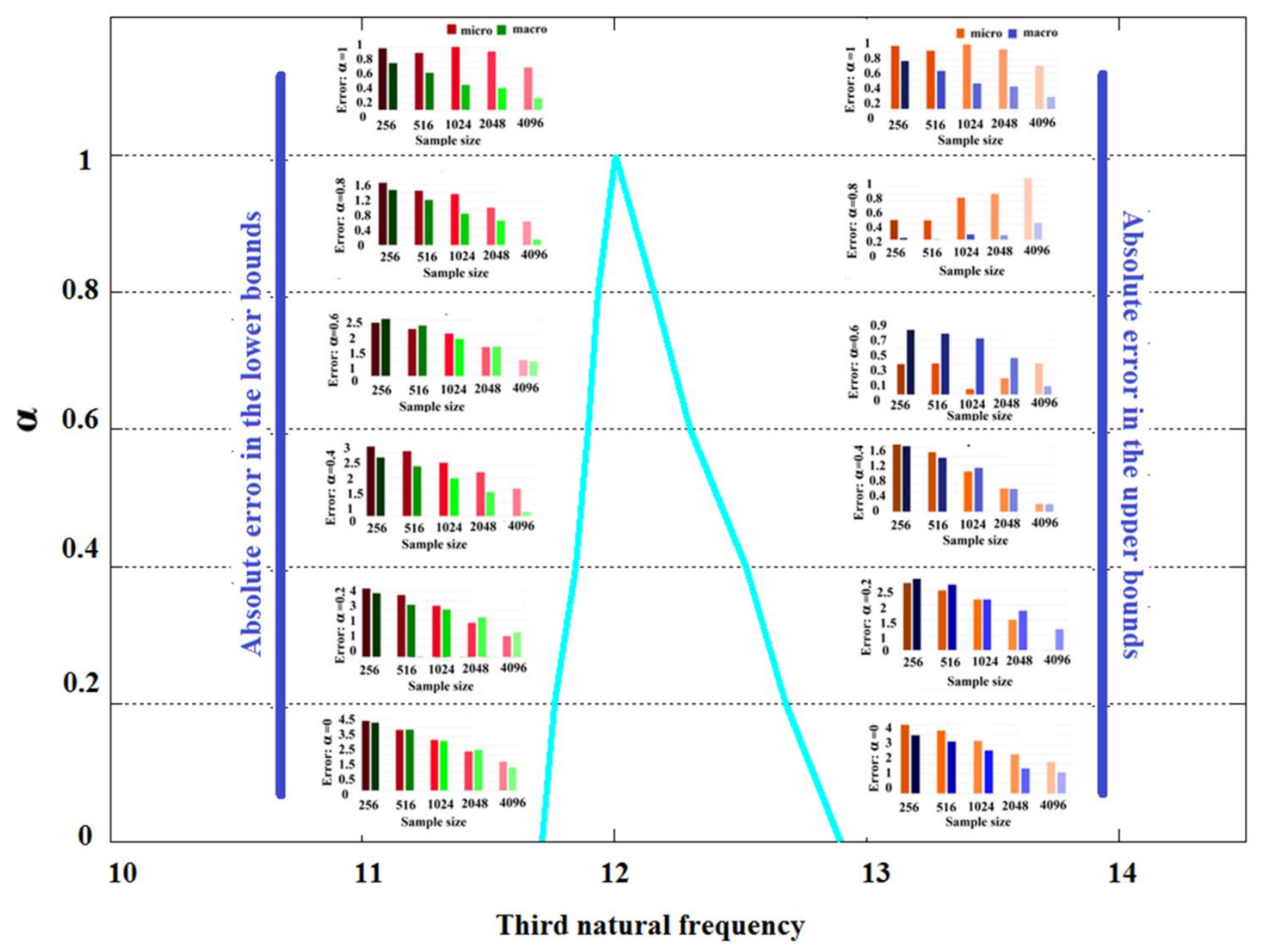

Fig. 11 Convergence study and validation for the sample size required to form the RBF model in case of third natural frequency $\left(g_{\text {micro }}^{C}\right.$ and $\left.g_{\text {macro }}^{C}\right)$. A typical membership function is shown considering the compound effect of stochasticity in micromechanical parameters $\left(g_{\text {micro }}^{C}\right)$.

finding the lower and upper bounds corresponding to each $\alpha$-cut. In this paper, the lower and upper bounds corresponding to each $\alpha$-cut are obtained based on 10,000 samples resulting in a total of 50 , 000 samples. Thus it yields to a level of computational efficiency of more than $1 / 12(\sim 4096 / 50000)$ times in terms of finite element simulations. It can be noted that the time taken for surrogate model formation and prediction is negligible compared to a single finite element simulation. Thus we have ignored the time taken for surrogate model formation and prediction, and reported the efficiency in terms of the number of finite element simulations required in this study.

\subsubsection{Numerical results for fuzzy dynamic analysis}

Having the finite element model and the RBF model validated, as shown in the preceding subsection, results for fuzzy dynamic analysis are presented in this subsection for the first three 


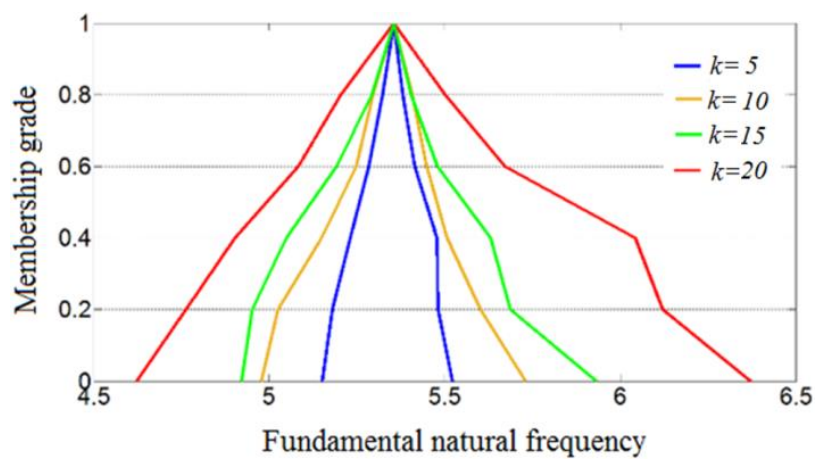

(a)

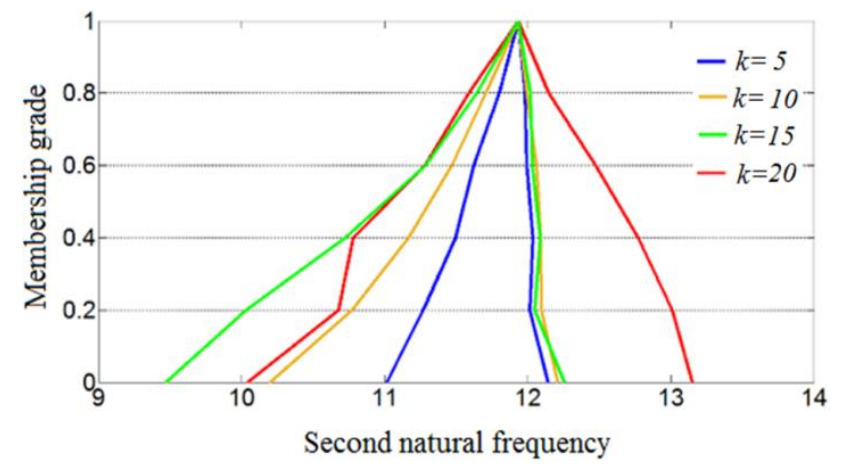

(b)

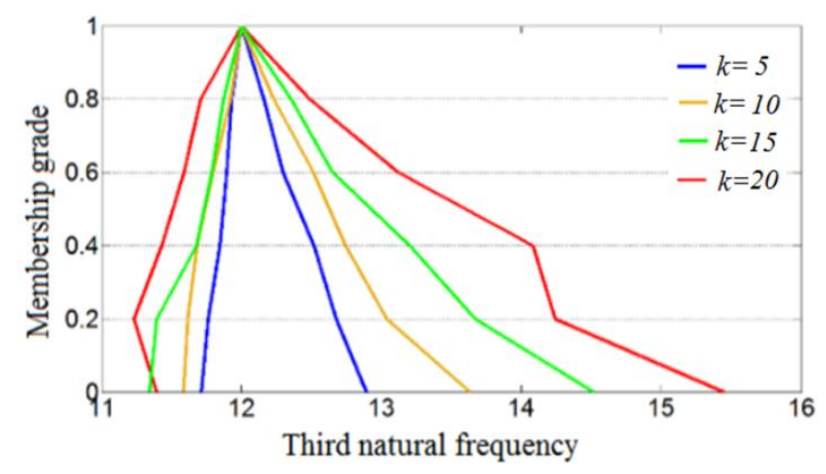

(c)

Fig. 12 Effect of variation in degree of fuzziness $(k)$ on the first three natural frequencies $\left(g_{\text {micro }}^{C}\right)$

modes of vibration considering a composite plate with spatially varying material properies $\left(g_{\text {macro }}^{C}\right.$ and $\left.g_{\text {micro }}^{C}\right)$. Figure 12 shows the variation of first three fuzzy natural frequencies considering different degrees of fuzziness corresponding to $\alpha=0$ with the fuzzy uncertainty at the micro-mechanical level. The response bounds are noticed to increase with the increasing degree of fuzziness. The fuzzy uncertainty for first three natural frequencies is investigated for various laminate configurations considering the $[\theta /-\theta / \theta]$ family of angle-ply composites and a cross-ply composite of configuration $\left[0^{\circ} / 90^{\circ} / 0^{\circ}\right]$ accounting spatially varying fuzzy source-uncertainty in the micromechanical properties. The fuzzy membership functions presented in figure 13 shows that the results corresponding to $\alpha=1$ varies depending on the effective stiffness of the structure, while the fuzzy bounds show different behaviour corresponding to different laminate configurations. Figure 14 shows the influence of boundary conditions on the fuzzy natural frequencies of composite plates for simply supported (SSSS) and fixed (CCCC) boundaries at all the four edges. The results corresponding to $\alpha=1$ are found to vary depending on the stiffness of the system, following a similar trend as deterministic 


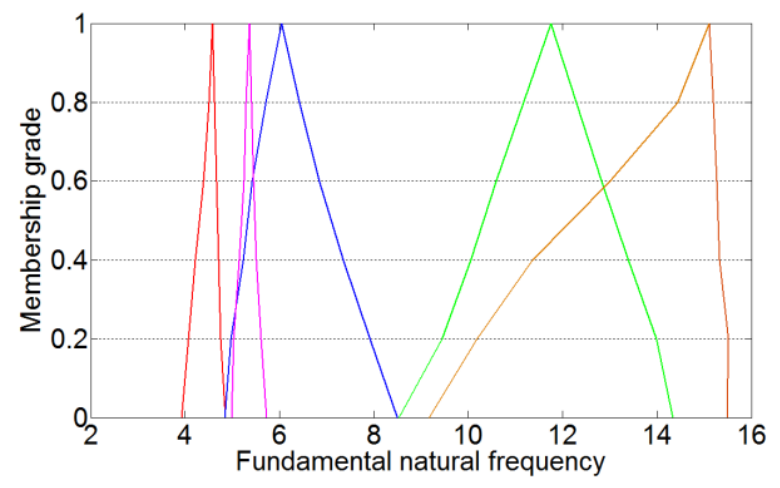

(a)

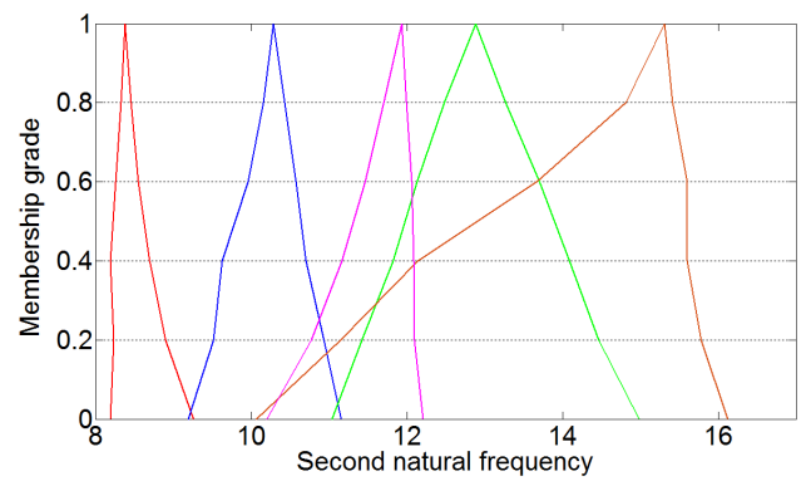

(b)

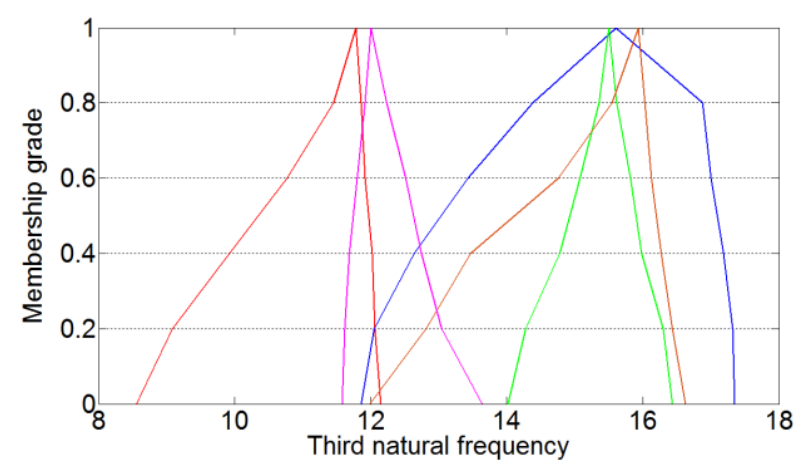

(c)

$$
\begin{aligned}
& -0^{\circ} / 0^{\circ} / 0^{\circ}-30^{\circ} /-30^{\circ} / 30^{\circ}-60^{\circ} /-60^{\circ} / 60^{\circ} \\
& -90^{\circ} /-90^{\circ} / 90^{\circ}-0^{\circ} / 90^{\circ} / 0^{\circ}
\end{aligned}
$$

Fig. 13 Effect of ply-orientation angle on the fuzzy natural frequencies of composite plates $\left(g_{\text {micro }}^{C}\right)$

analysis. Figure 15 shows the effect of aspect ratio of the composite laminated plates on the fuzzy natural frequencies considering stochastic micromechanical material properties. The fuzzy natural frequencies are noticed to reduce with the increase in aspect ratio for all the three natural frequencies along with a reduction in fuzzy-bounds. Figure 16 shows the effect of change in degree of orthotropy on the fuzzy natural frequencies considering source-uncertainties in the macromechanical properties. The source-uncertainty in the macromechanical properties are considered in this case because of the direct definition of degree of orthotropy as a ratio of longitudinal and transverse Young's modulus at macro-scale. The natural frequencies are found to increase with increasing degree of orthotropy for all the three considered vibration modes. Comparative results considering the fuzzy sourceuncertainty at the micro and macro mechanical levels are presented in figure 17 considering two different boundary conditions. To understand the relative effect of fuzzy uncertainty, the results in this figure are normalized with respect to their respective deterministic values. 


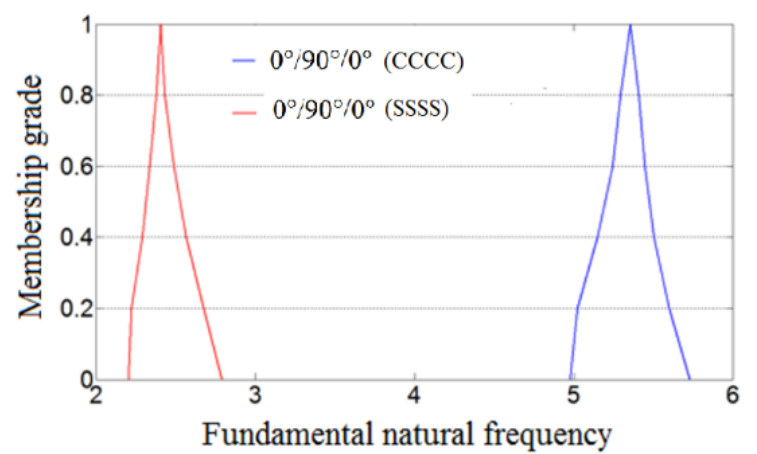

(a)

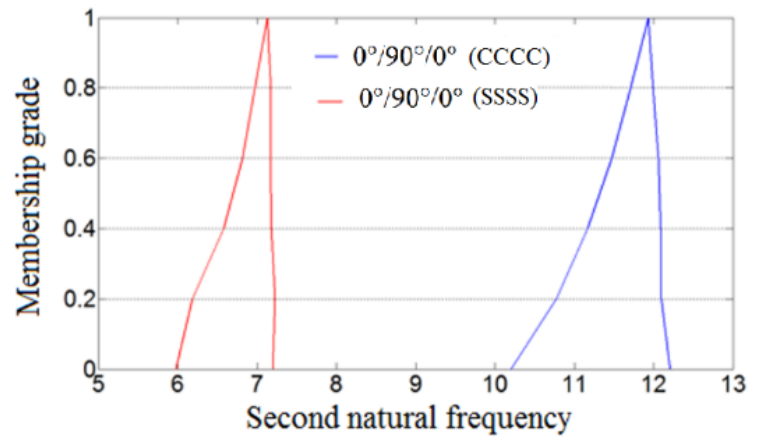

(b)

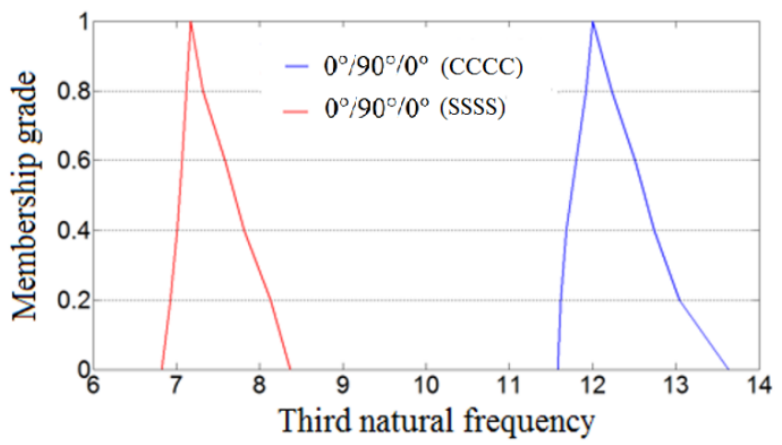

(c)

Fig. 14 Effect of boundary condition on the fuzzy natural frequencies of cross-ply composite plates with $\left[0^{\circ} / 90^{\circ} / 0^{\circ}\right]$ configuration $\left(g_{\text {micro }}^{C}\right)$

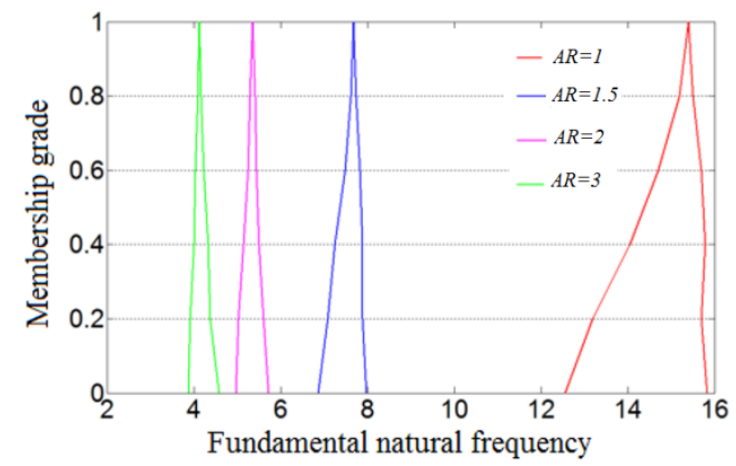

(a)

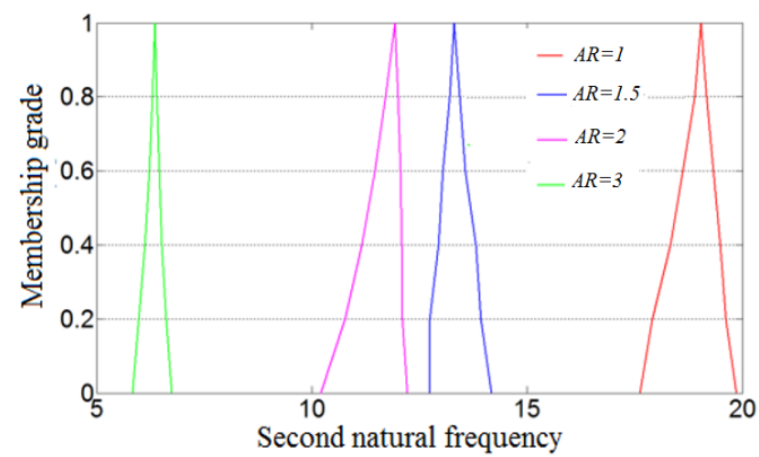

(b)

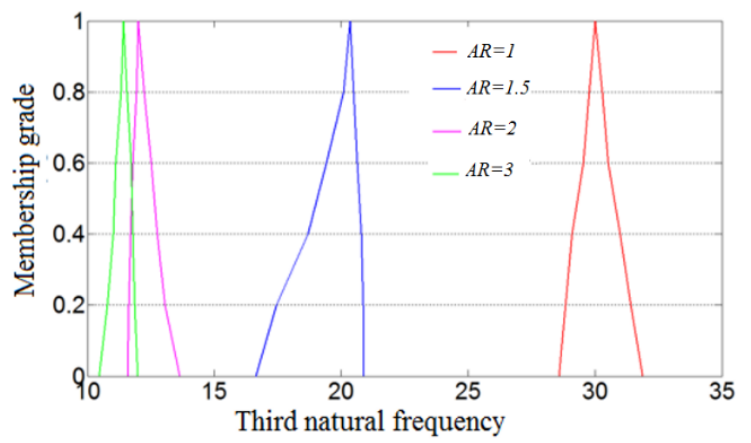

(c)

Fig. 15 Effect of aspect ratio $(A R)$ on the fuzzy natural frequencies of composite plates $\left(g_{\text {micro }}^{C}\right)$ with fixed boundary condition 


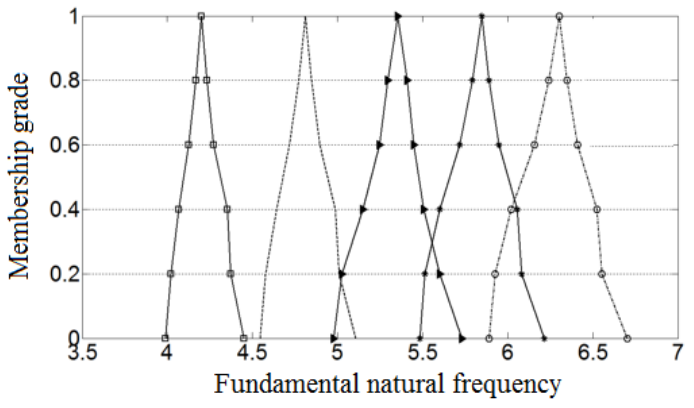

(a)

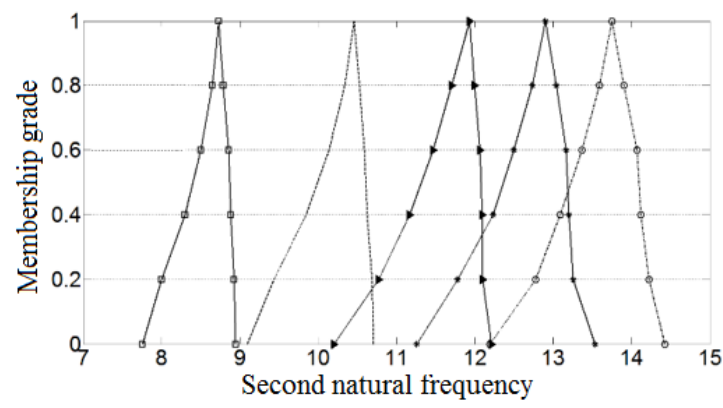

(b)

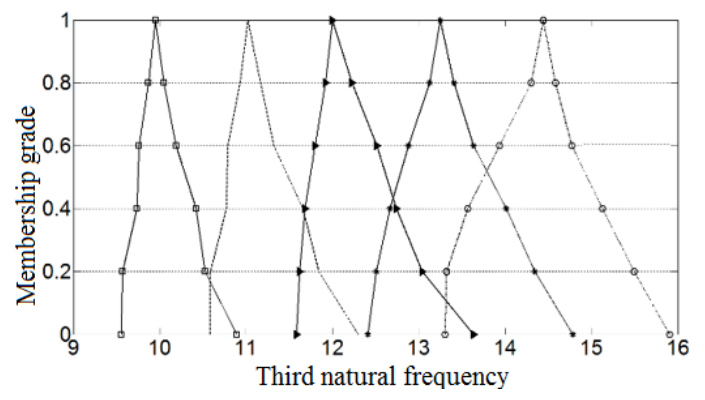

(c)

$$
\begin{aligned}
& \rightarrow D O=20 \rightarrow D O=40 \\
& \rightarrow D O=30 \rightarrow D O=50
\end{aligned} \rightarrow D O=60
$$

Fig. 16 Effect of degree of orthotropy $(D O)$ on the fuzzy natural frequencies of composite plates $\left.g_{\text {macro }}^{C}\right)$

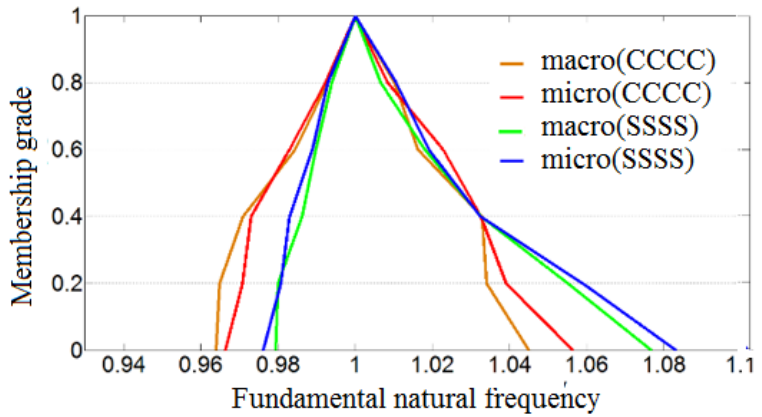

(a)

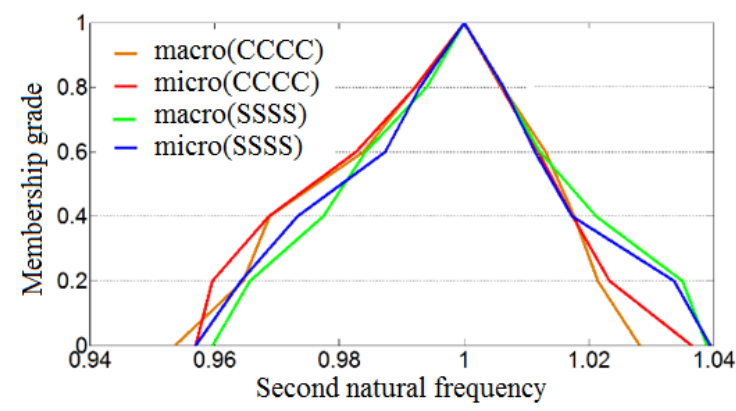

(b)

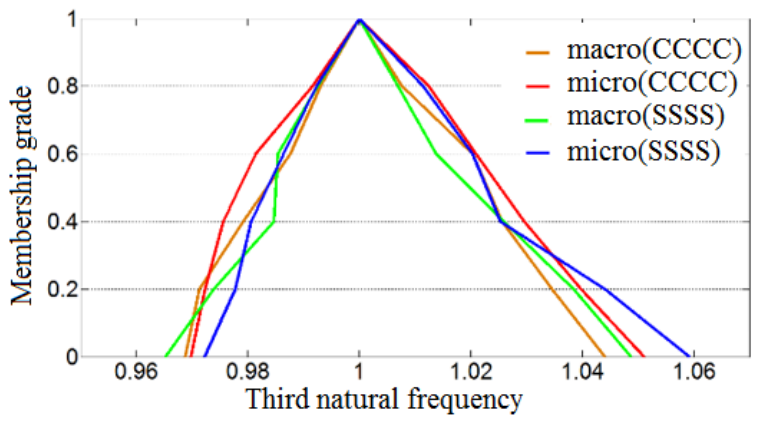

(c)

Fig. 17 Comparative results for natural frequencies considering micromechanical $\left(g_{\text {micro }}^{C}\right)$ and macromechanical analyses $\left(g_{\text {macro }}^{C}\right.$ ) of cross-ply composite plates 


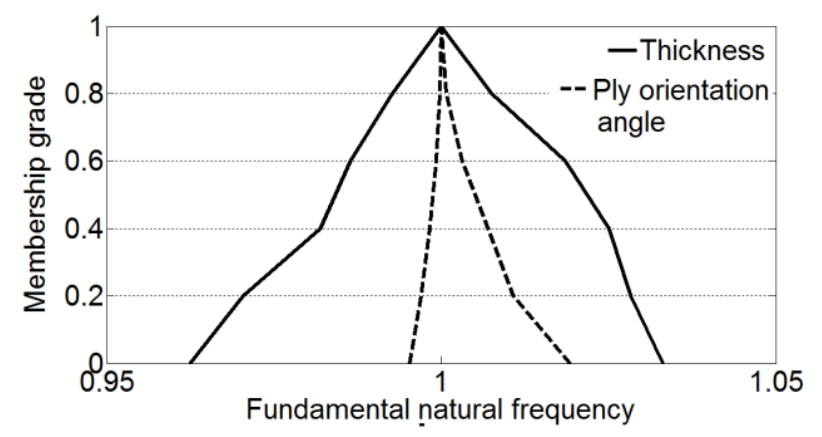

(a)

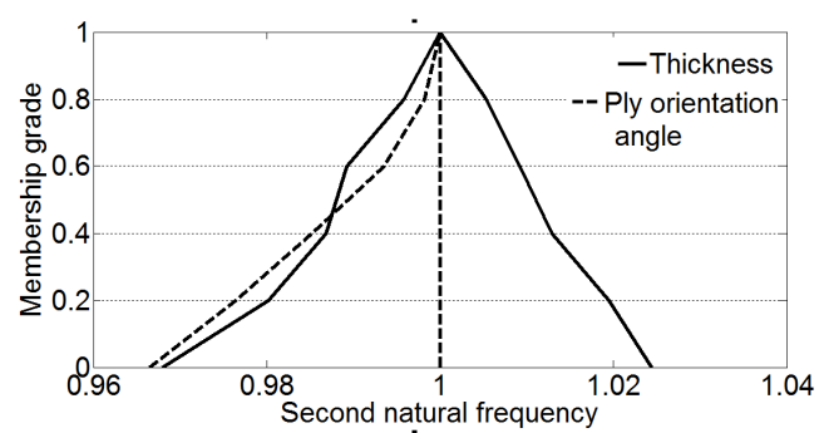

(b)

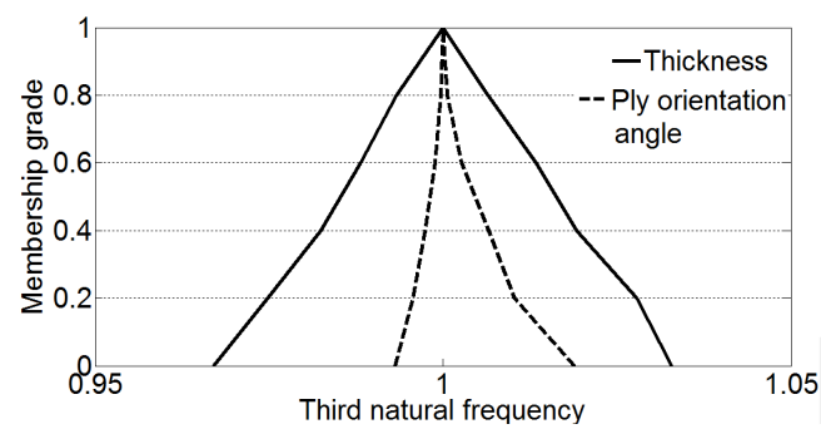

(c)

Fig. 18 Individual effect of fuzzy uncertainty in the geometric properties $\left(g_{G}^{I}\right)$ of composite plates (Normalized results are presented with respect to the respective deterministic natural frequencies)

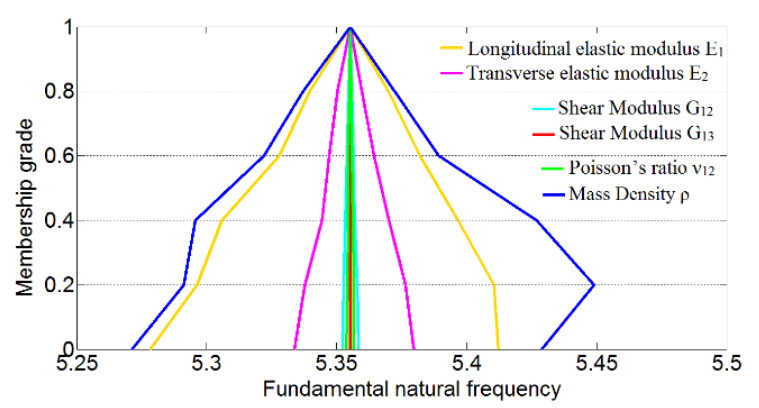

(a)

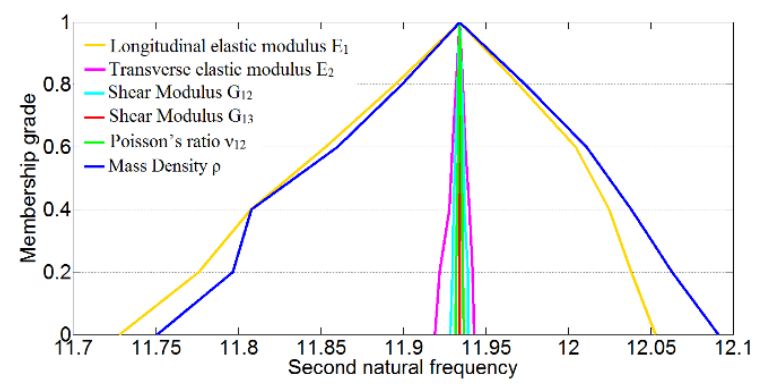

(b)

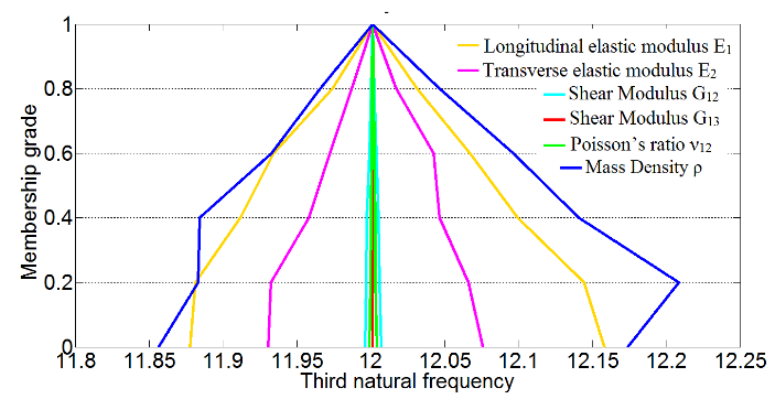

(c)

Fig. 19 Individual effect of fuzzy uncertainty in the macromechanical $\left(g_{\text {macro }}^{I}\right)$ properties (Normalized results are presented with respect to the respective deterministic natural frequencies) 


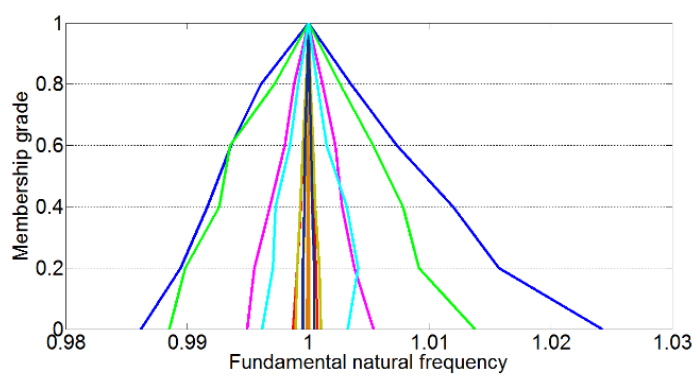

(a)

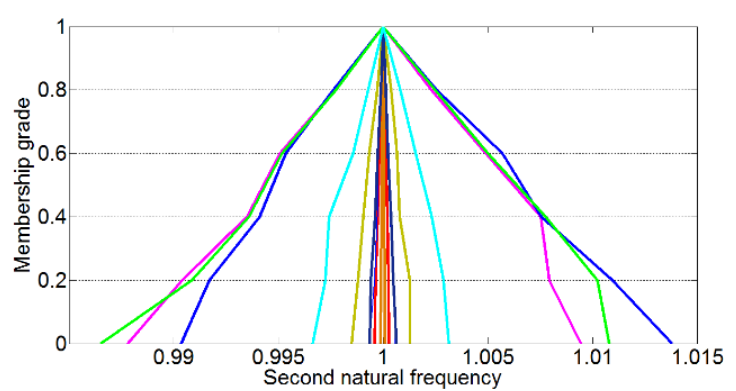

(b)

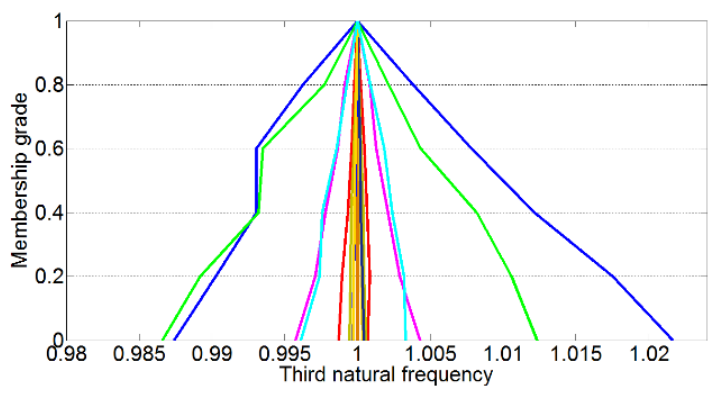

(c)

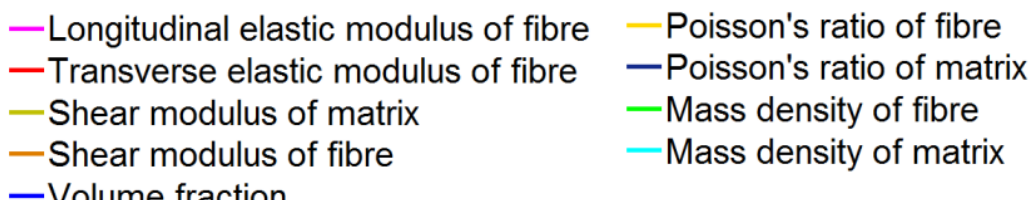

- Volume fraction

Fig. 20 Individual effect of fuzzy uncertainty in the micromechanical properties $\left(g_{\text {micro }}^{I}\right)$

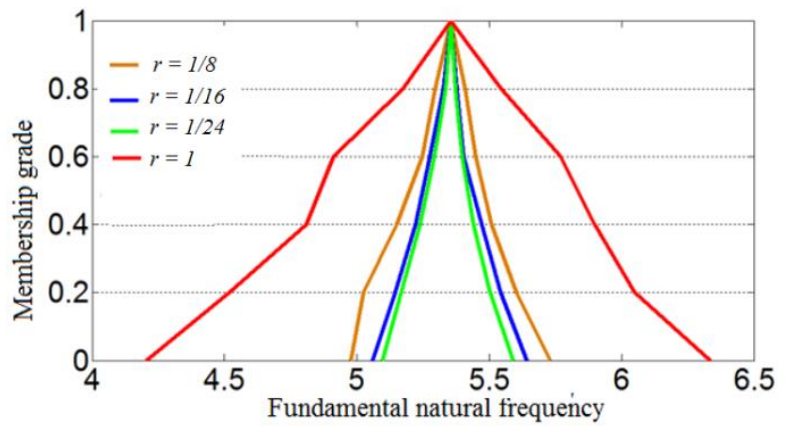

(a)

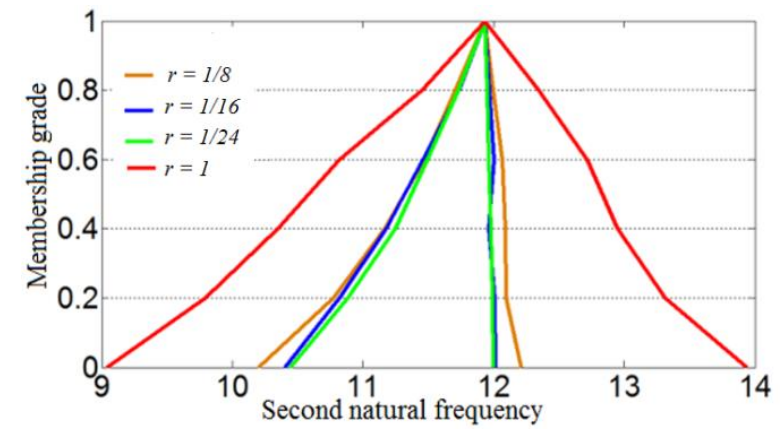

(b)

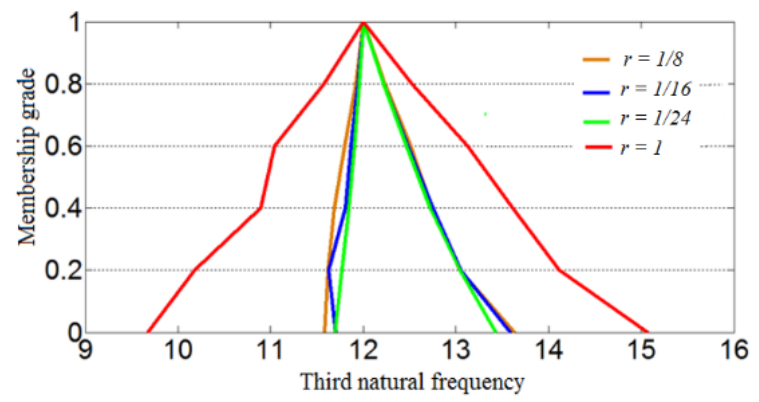

(c)

Fig. 21 Effect of characteristic length $(r)$ on the fuzzy natural frequencies $\left(g_{\text {micro }}^{C}\right)$ 
The results in this subsection so far are presented considering the compound effect of fuzzy uncertainty at micro and macro-mechanical level. Figure $18-20$ show the individual effect of fuzzy source-uncertainties in micro and macro mechanical properties along with the geometric attributes. Depending on the respective fuzzy response bounds, these figures can provide a clear idea regarding the sensitivity of various individual material and geometric parameters to the fuzzy natural frequencies of the composite plate. The nature of fuzzy random field of the stochastic micro and macro mechanical material properties depend on the characteristic length $(r)$ considered in the analysis. A higher value of $r$ indicates less rapid variation of the material property concerned and vice versa. We have investigated the effect of characteristic length on the first three modes of vibration. It can be noted that the system becomes a randomly homogenous system (analogous to considering no spatial variation [Dey et al. (2016b)] when the characteristic length $r \geq 1$. The effect of characteristic length on the first three fuzzy natural frequencies is presented in figure 21 , wherein a clear difference is noticed between the case of considering no spatial variation and the present analysis considering spatially varying attributes. The results reveal that the conventional practise [Dey et al. (2016b)] of neglecting the inevitable spatial variation in material properties overestimates the fuzzy response bounds significantly.

The effect of fuzzy source-uncertainty in micro and macro mechanical material properties are studied on the vibration mode shapes considering two different boundary conditions (SSSS and CCCC). The results are presented in figure 22 for first three modes of vibration. Fuzzy mode shapes considering a single random realization are presented in case of: a clamped (CCCC) composite plate with stochasticity in the macromechanical $\left(g_{\text {macro }}^{C}\right)$ properties (refer to figure $22(\mathrm{a}-\mathrm{c})$ ), a simply supported (SSSS) composite plate with stochasticity in the macromechanical $\left(g_{\text {macro }}^{C}\right)$ properties (refer to figure 22(d - f)), a clamped (CCCC) composite plate with stochasticity in the micromechanical $\left(g_{\text {micro }}^{C}\right)$ properties (refer to figure $22(\mathrm{~g}-\mathrm{i})$ ) and a clamped $(\mathrm{CCCC})$ composite plate with stochasticity in the micromechanical $\left(g_{\text {micro }}^{C}\right)$ properties (refer to figure $22(\mathrm{j}-1)$ ). From the mode shapes presented in figure 22(a-1), it can be observed that the basic global pattern of the 


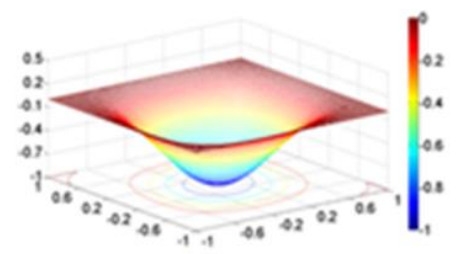

(a)

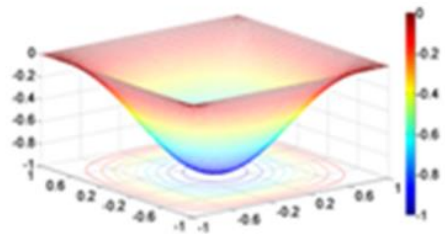

(d)

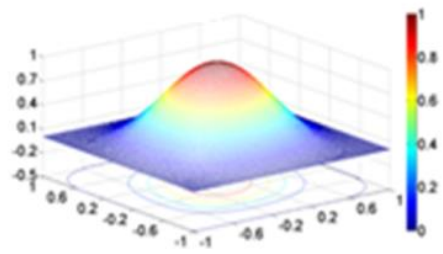

(g)

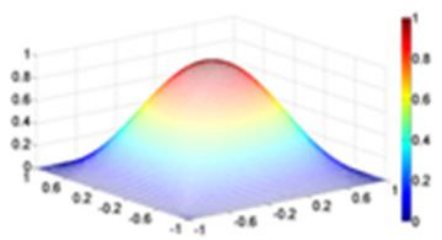

(j)

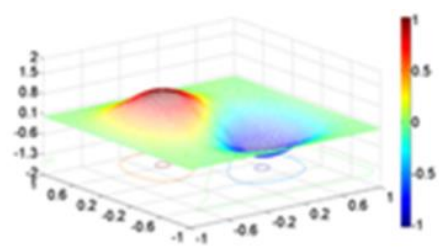

(b)

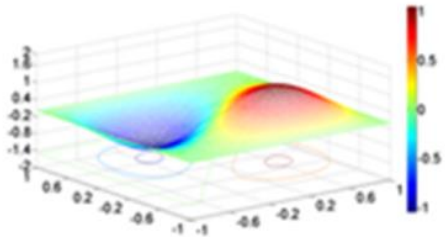

(e)

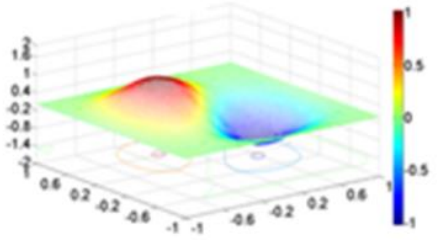

(h)

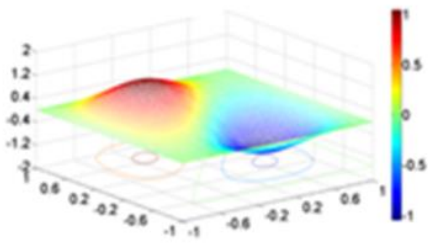

(k)

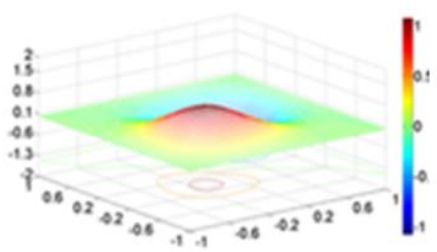

(c)

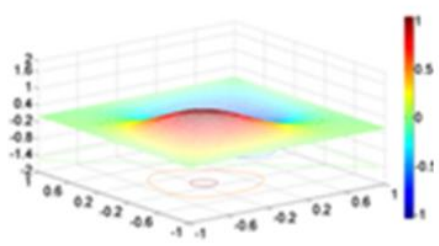

(f)

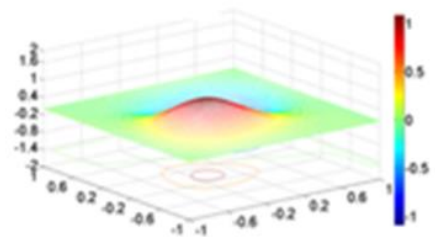

(i)

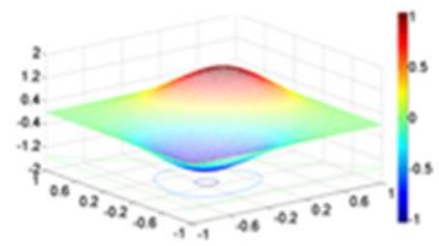

(1)

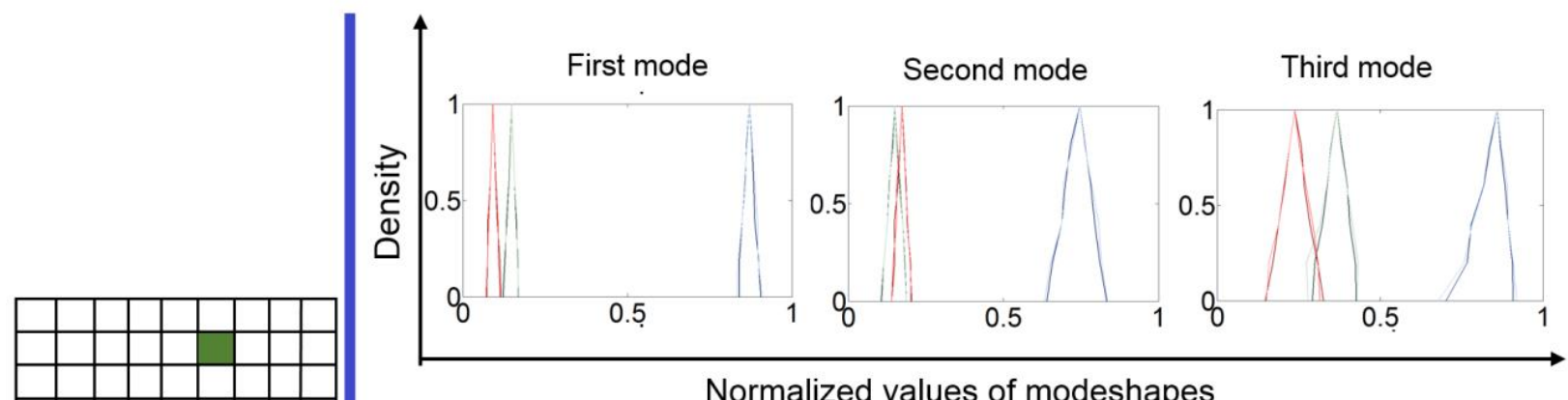

(m)

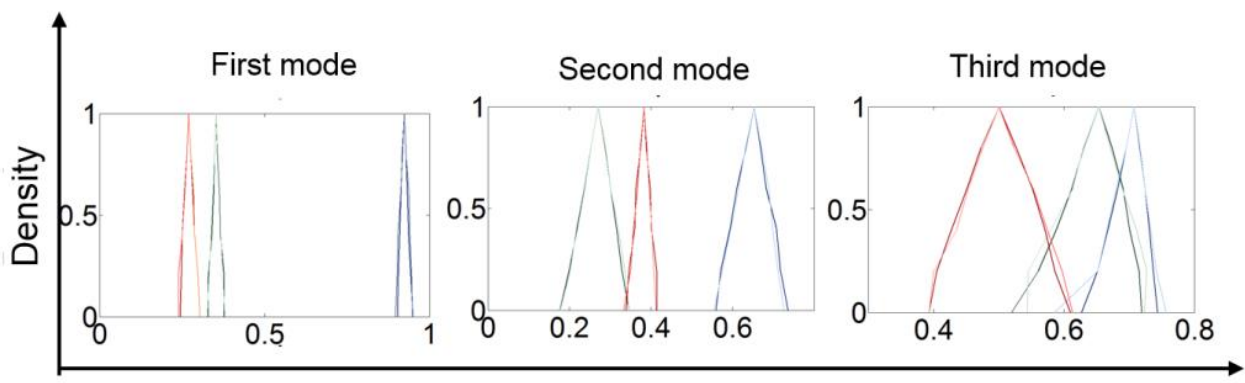

Normalized values of modeshapes

(n)

Fig. 22 Fuzzy modeshapes and representative membership functions of the normalized eigenvectors ( $g_{\text {micro }}^{C}$ and $g_{\text {macro }}^{C}$ ) 
stochastic mode shapes remains similar to the corresponding deterministic case. However, the value of normalized eigenvectors becomes fuzzy in nature for each of the elements in the composite plate. Fuzzy membership functions of the normalized eigenvectors of first three vibration modes for the elements indicated in figure 22(o) are shown considering a clamped (CCCC) (refer to figure 22(m)) and a simply supported (SSSS) (refer to figure 22(n)) boundary conditions. The results for micro and macro mechanical analyses are shown using lighter and darker shades of respective colours indicated in figure 12(o). It can be noticed that the fuzzy membership functions depend significantly on the type of analysis (micro and macro mechanical) and location of the element under consideration.

\subsection{Fuzzy stability analysis}

\subsubsection{Validation and convergence study}

The finite element code and the RBF model are validated first for analysing the buckling loads similar to the case of dynamic analysis. The results of convergence study and validation of the finite element code of a composite plate are furnished in figure 23, wherein the non-dimensional first buckling load is validated with the results available in scientific literature. Based on the results presented in the figure, a mesh size of $9 \times 9$ is found to be adequate for the finite element model. The optimum number of samples (drawn from Sobol sequence) to form the surrogate models of buckling loads are decided based on a comparative performance similar to the case of dynamic analysis presented in the preceding subsection. The results for macro and micro mechanical analyses showing the values of absolute error for different sample sizes corresponding to different $\alpha$-cuts are presented in figure 24 - 26. From the figures it is evident that a sample size of 4096 provides reasonably accurate results for the first three buckling loads. It can be noted in this context that the RBF model for a buckling load is formed only for $\alpha=0$, while the same RBF model can be used for prediction corresponding to the other $\alpha$-cuts. In this subsection, we have presented the results of stochastic stability analysis considering the first three buckling modes for the sake of completeness. Besides that, in case of fuzzy stability analysis, the buckling loads are found to have a fuzzy response bound with respect to the corresponding deterministic values. Thus there exists a possibility of overlap in the fuzzy responses of the buckling loads corresponding to different modes of buckling resulting in a 


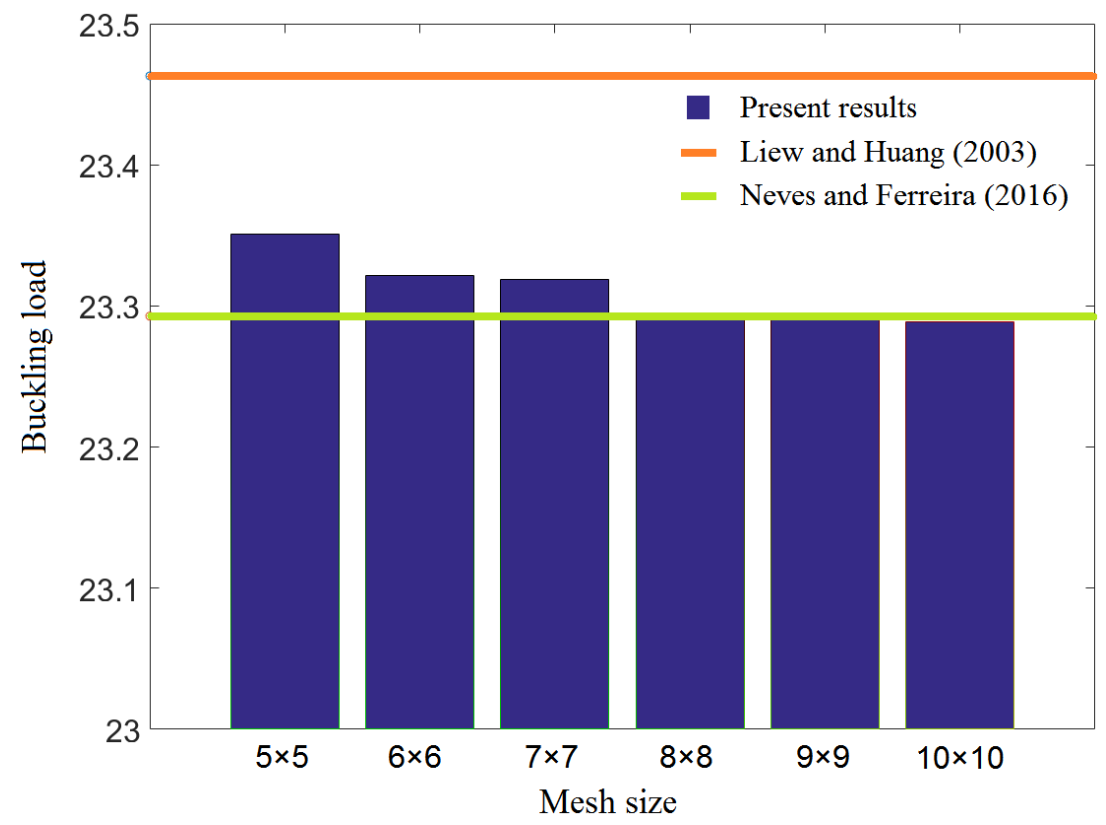

Fig. 23 The convergence study of uniaxial buckling load of four layer $\left(0^{\circ} / 90^{\circ} / 90^{\circ} / 0^{\circ}\right)$ simply supported (SSSS) rectangular laminates with respect to Liew and Huang (2003) and Neves and Ferreira (2016)

non-unique critical buckling mode with the minimum value of buckling load. For this reason, it is essential to consider higher buckling modes in case of fuzzy-uncertainty in the system parameters.

\subsubsection{Numerical results for fuzzy stability analysis}

Numerical results are presented in this subsection for the first three modes of buckling. Figure 27 shows the variation of first three fuzzy buckling loads considering different degrees of fuzziness corresponding to $\alpha=0$ with the fuzzy uncertainty at the micro-mechanical level. The response bounds are noticed to increase with the increasing degree of fuzziness. The effect of fuzzy uncertainty for first three buckling loads is investigated for various laminate configurations considering the $[\theta /-\theta / \theta]$ family of angle-ply composites and a cross-ply composite of configuration $\left[0^{\circ} / 90^{\circ} / 0^{\circ}\right]$ accounting spatially varying fuzzy source-uncertainty in the micromechanical properties. The fuzzy membership functions presented in figure 28 indicate that the results corresponding to $\alpha=1$ varies depending on the effective stiffness of the structure, while the fuzzy bounds show different behaviour corresponding to different laminate configurations. Figure 29 shows the influence of boundary conditions on the fuzzy buckling loads of composite plates for simply supported (SSSS) and fixed (CCCC) boundaries at all the four edges. The results corresponding to $\alpha=1$ are found to vary depending on the stiffness of the system, following a similar trend as deterministic analysis. Figure 


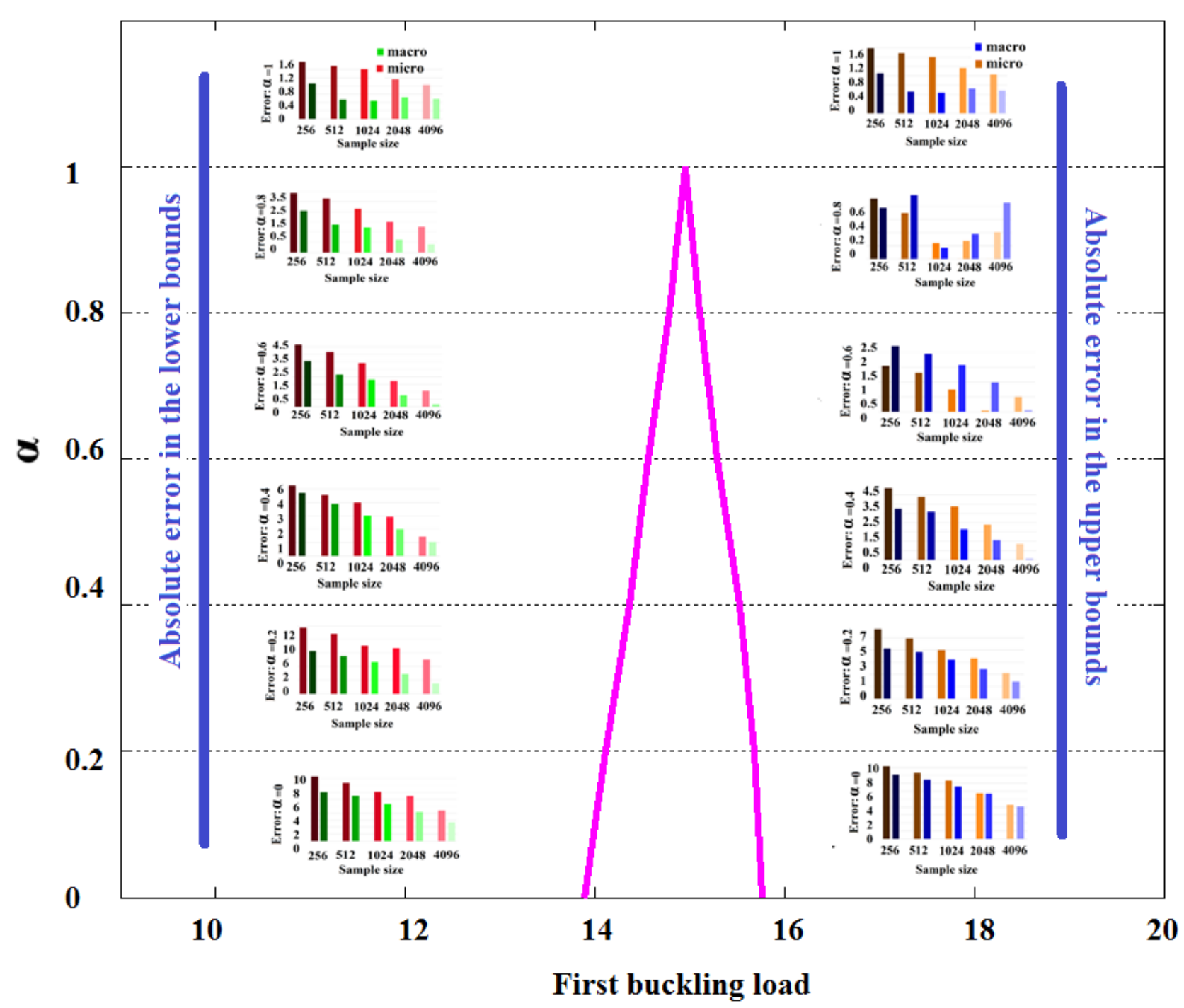

Fig. 24 Convergence study and validation for the sample size required to form the RBF model in case of first buckling load ( $g_{\text {micro }}^{C}$ and $g_{\text {macro }}^{C}$ ). A typical membership function is shown considering the compound effect of stochasticity in micromechanical parameters $\left(g_{\text {micro }}^{C}\right)$.

30 shows the effect of aspect ratio of the composite laminated plates on the fuzzy buckling loads considering stochastic micromechanical material properties. The fuzzy buckling loads are noticed to reduce with the increase in aspect ratio for all the three modes of buckling along with a reduction in fuzzy-bounds. Figure 31 shows the effect of change in degree of orthotropy on the fuzzy buckling loads considering source-uncertainties in the macromechanical properties. The first three buckling loads are found to increase with increasing degree of orthotropy. Comparative results considering the fuzzy source-uncertainty at the micro and macro mechanical levels are presented in figure 32 considering two different boundary conditions. To understand the relative effect of fuzzy uncertainty, the results in this figure are normalized with respect to their respective deterministic values. 


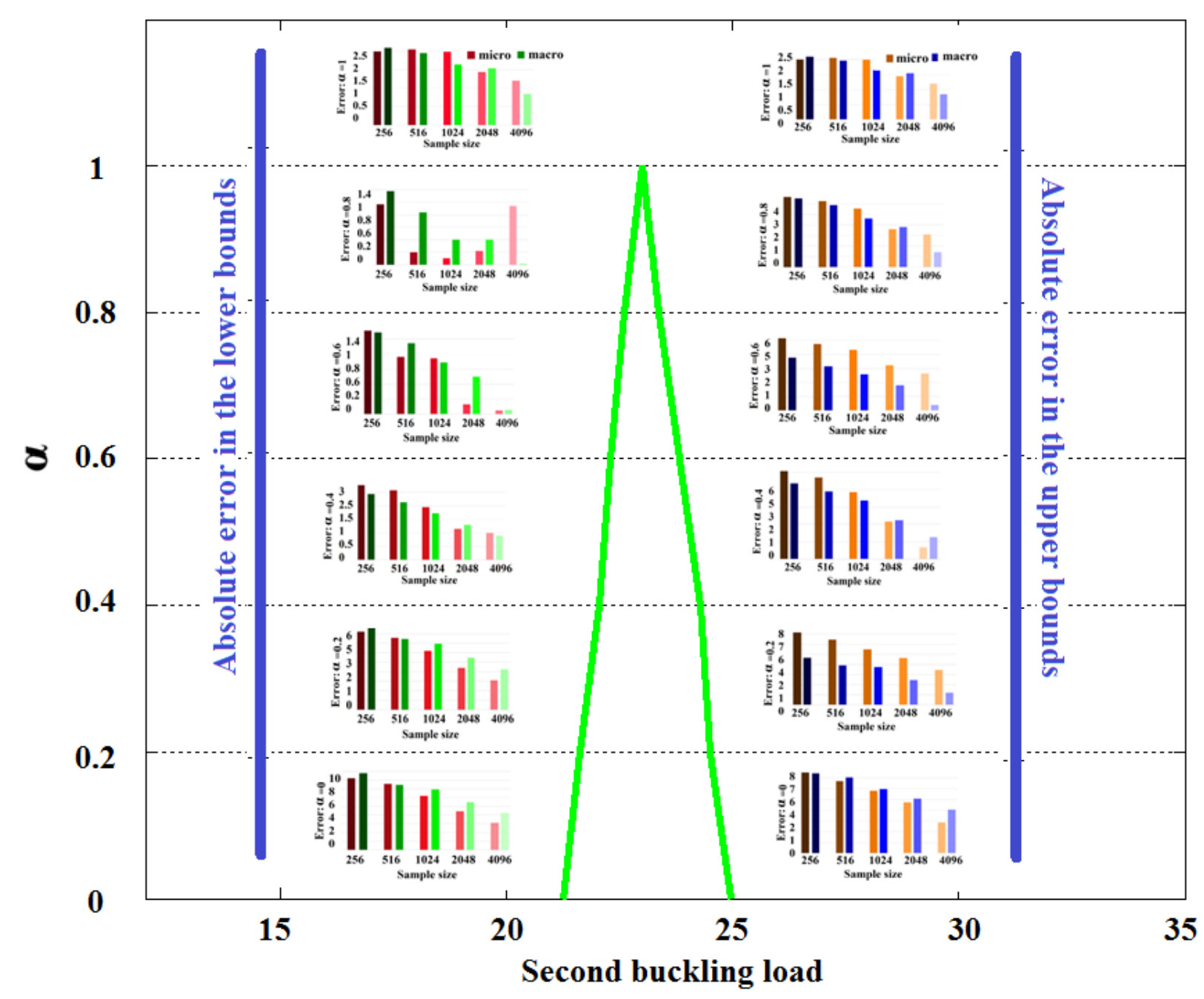

Fig. 25 Convergence study and validation for the sample size required to form the RBF model in case of second buckling load ( $g_{\text {micro }}^{C}$ and $g_{\text {macro }}^{C}$ ). A typical membership function is shown considering the compound effect of stochasticity in micromechanical parameters $\left(g_{\text {micro }}^{C}\right)$.

Figure 33 - 35 show the individual effect of fuzzy source-uncertainties in micro and macro mechanical properties along with the geometric attributes. Depending on the respective fuzzy response bounds, these figures can provide a clear idea regarding the sensitivity of various individual material and geometric parameters to the fuzzy buckling loads of the composite plate. The nature of fuzzy random field of the stochastic micro and macro mechanical material properties depend on the characteristic length $(r)$ considered in the analysis. Similar to the case of dynamic analysis, we have investigated the effect of characteristic length on the first three modes of buckling. It can be noted that the system becomes a randomly homogenous system (analogous to considering no spatial variation [Dey et al. (2016b)] when the characteristic length $r \geq 1$. The effect of characteristic length on the first three fuzzy buckling loads is presented in figure 36, wherein a clear difference is noticed 


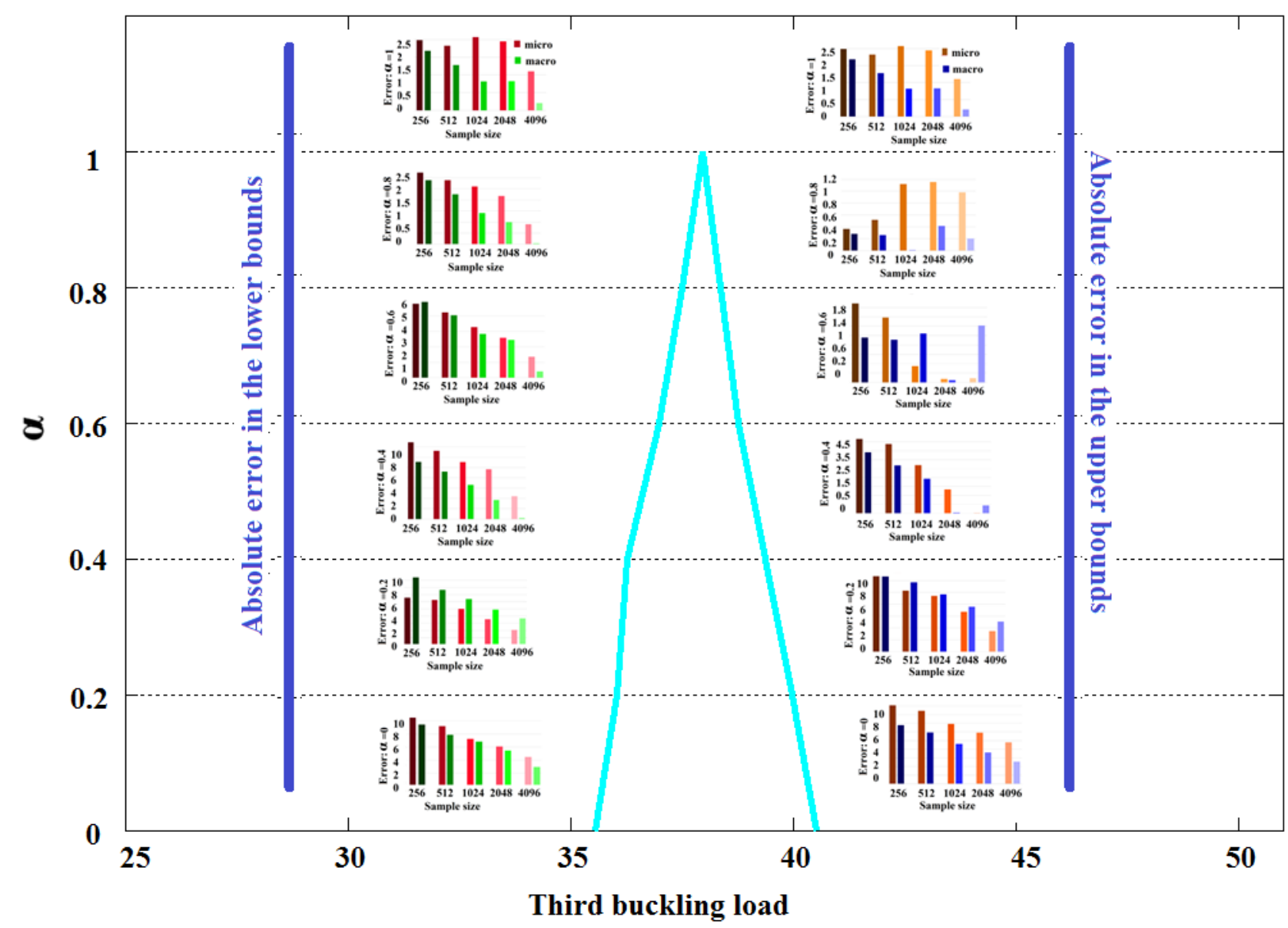

Fig. 26 Convergence study and validation for the sample size required to form the RBF model in case of third buckling load ( $g_{\text {micro }}^{C}$ and $g_{\text {macro }}^{C}$ ). A typical membership function is shown considering the compound effect of stochasticity in micromechanical parameters $\left(g_{\text {micro }}^{C}\right)$.

between the case of considering no spatial variation and the present analysis considering spatially varying attributes. The results reveal that the conventional practise of neglecting the inevitable spatial variation in material properties over-estimates the fuzzy response bounds of the buckling loads. The effect of fuzzy uncertainty in micro and macro mechanical attributes is studied on the buckling mode shapes considering two different boundary conditions (SSSS and CCCC). The results are presented in figure 37 for first three modes of buckling. Fuzzy mode shapes considering a single random realization are presented in case of: a clamped (CCCC) composite plate with stochasticity in the macromechanical $\left(g_{\text {macro }}^{C}\right)$ properties (refer to figure $37(\mathrm{a}-\mathrm{c})$ ), a simply supported (SSSS) composite plate with stochasticity in the macromechanical $\left(g_{\text {macro }}^{C}\right)$ properties (refer to figure $37(\mathrm{~d}-$ f)), a clamped (CCCC) composite plate with stochasticity in the micromechanical $\left(g_{\text {micro }}^{C}\right)$ properties 


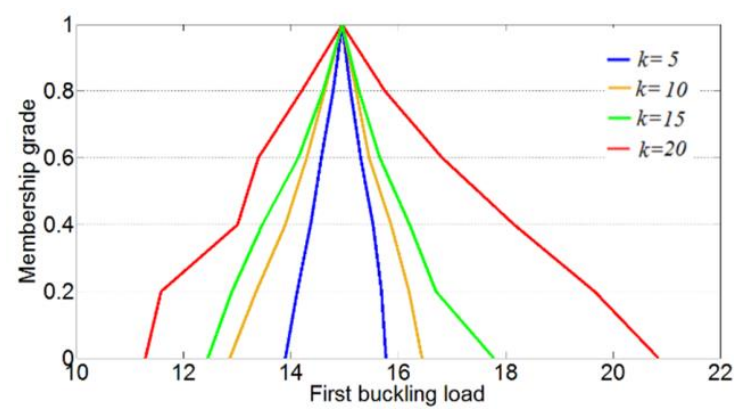

(a)

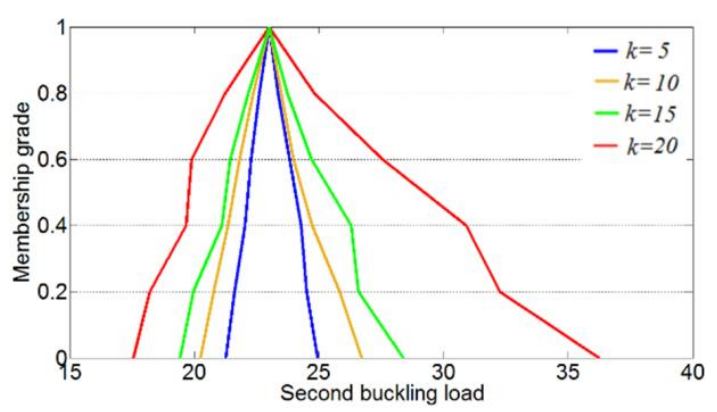

(b)

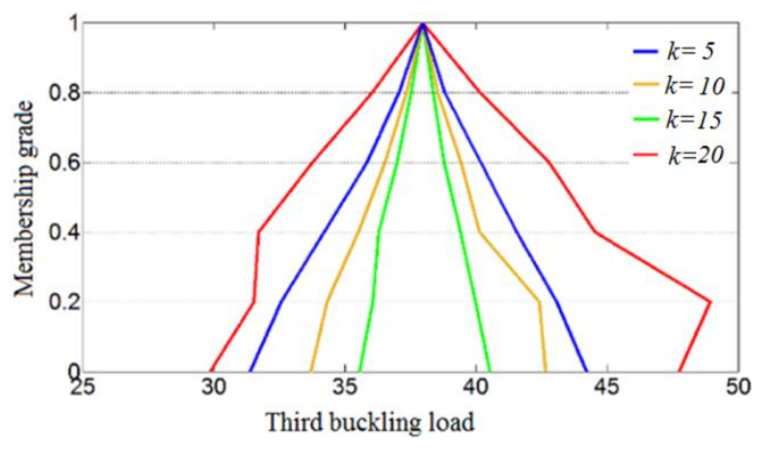

(c)

Fig. 27 Effect of variation in degree of fuzziness on the first three buckling loads $\left(g_{\text {micro }}^{C}\right)$

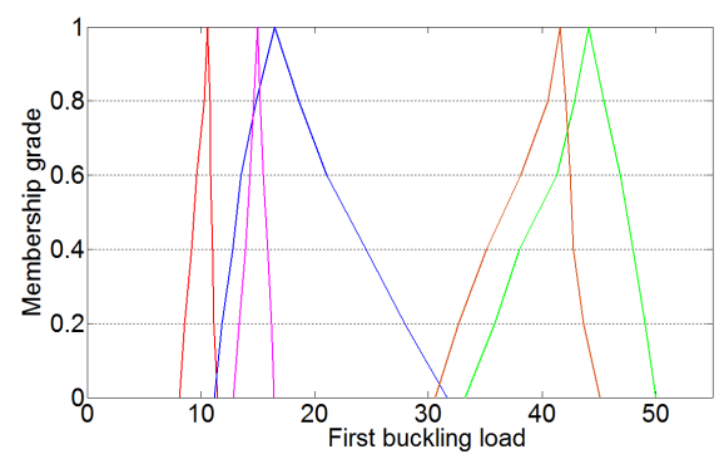

(a)

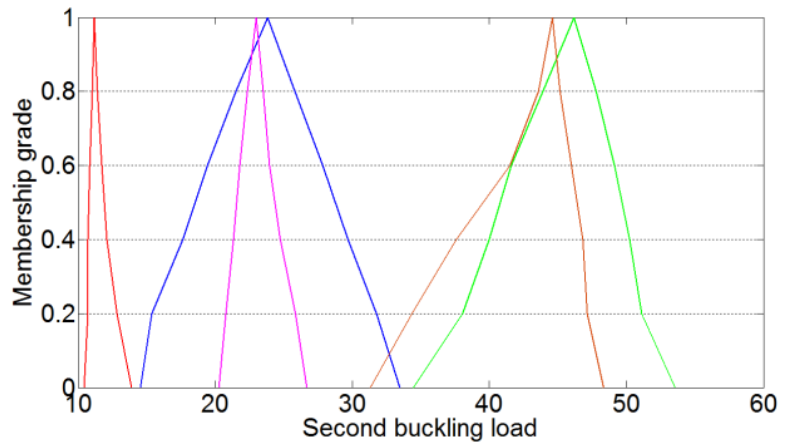

(b)

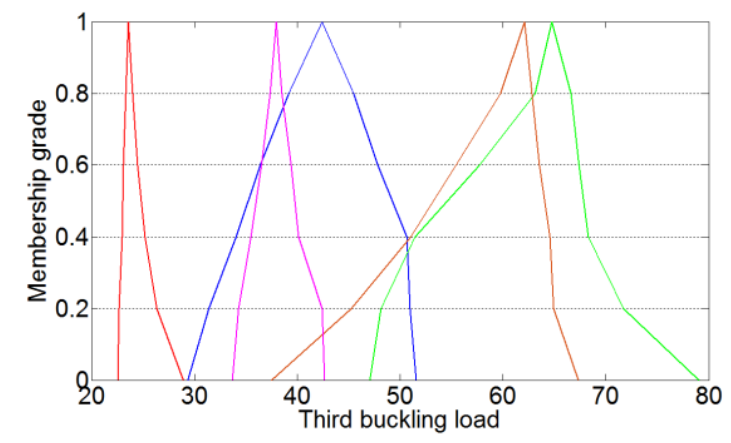

(c)

$$
\begin{gathered}
-0^{\circ} / 0^{\circ} / 0^{\circ}-30^{\circ} /-30^{\circ} / 30^{\circ}-60^{\circ} /-60^{\circ} / 60^{\circ} \\
-90^{\circ} / 90^{\circ} / 90^{\circ}-0^{\circ} / 90^{\circ} / 0^{\circ}
\end{gathered}
$$

Fig. 28 Effect of ply-orientation angle on the fuzzy buckling loads of composite plates $\left(g_{\text {micro }}^{C}\right)$ 


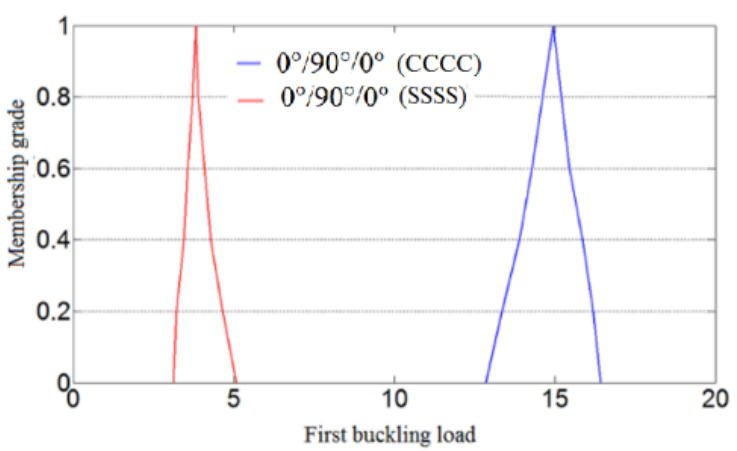

(a)

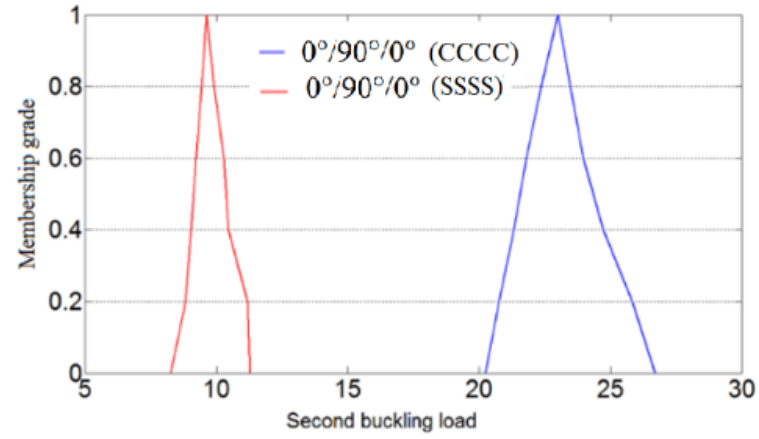

(b)

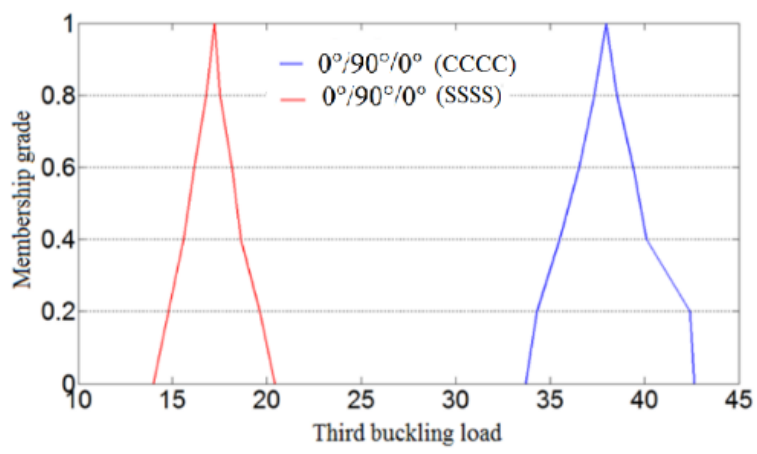

(c)

Fig. 29 Effect of boundary condition on the fuzzy buckling loads of cross-ply composite plates with $\left[0^{\circ} / 90^{\circ} / 0^{\circ}\right]$ configuration $\left(g_{\text {micro }}^{C}\right)$

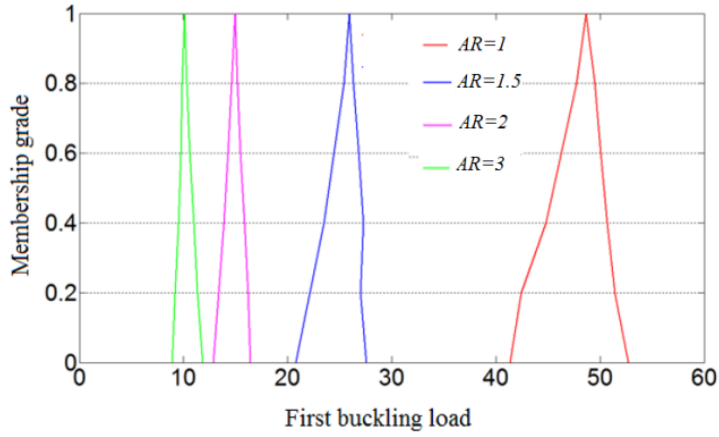

(a)

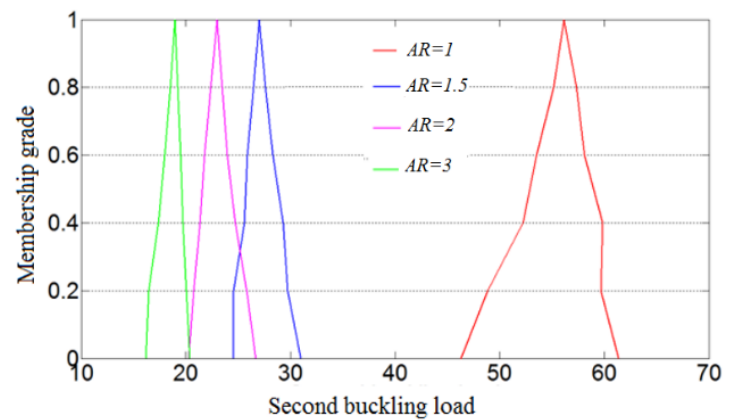

(b)

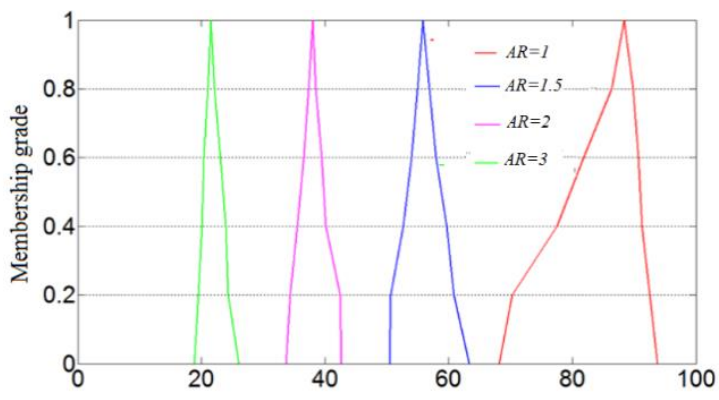

(c)

Fig. 30 Effect of aspect ratio $(A R)$ on the fuzzy buckling loads of composite plates $\left(g_{\text {micro }}^{C}\right)$ 


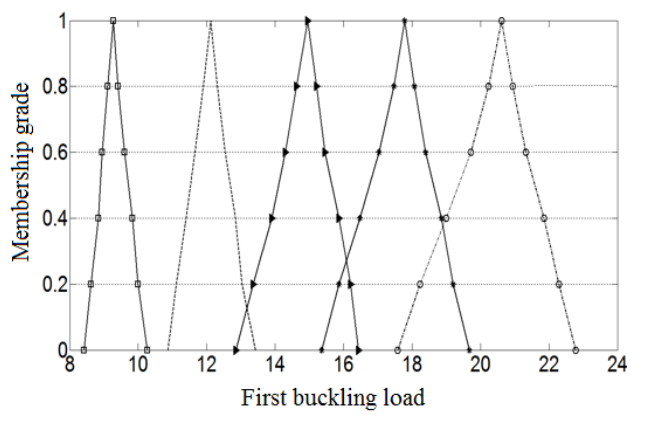

(a)

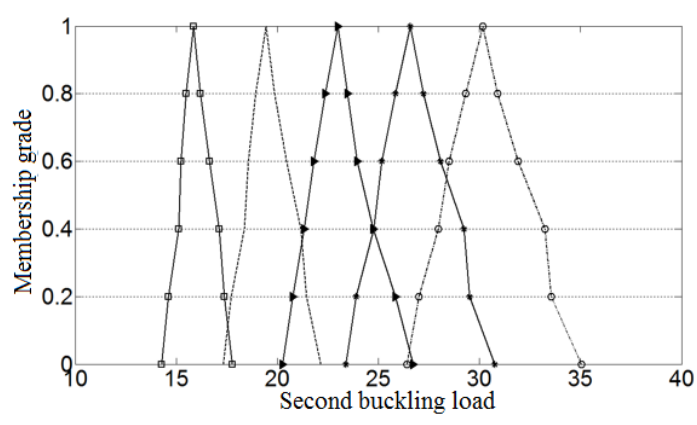

(b)

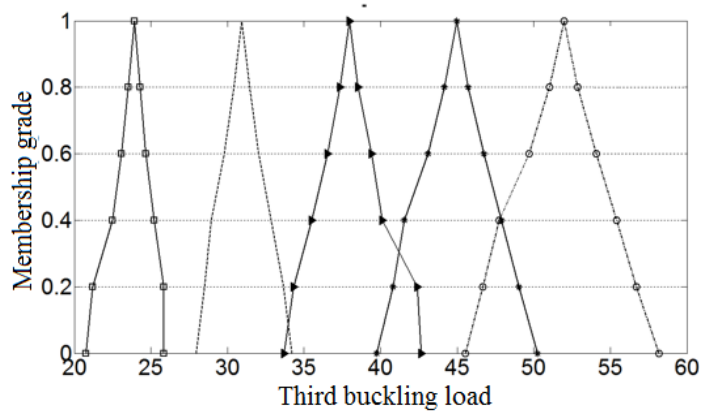

(c)

$$
\begin{aligned}
& \rightarrow D O=20 \rightarrow D O=40 \\
& \rightarrow D O=30 \rightarrow D O=50
\end{aligned} \rightarrow D O=60
$$

Fig. 31 Effect of degree of orthotropy $(D O)$ on the fuzzy buckling loads of composite plates $\left(g_{\text {micro }}^{C}\right)$

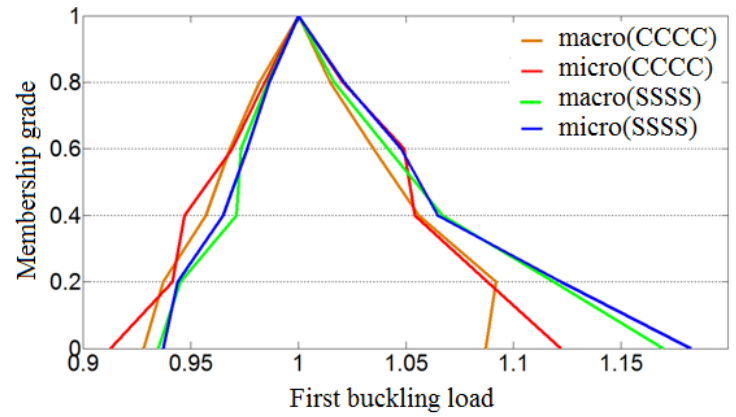

(a)

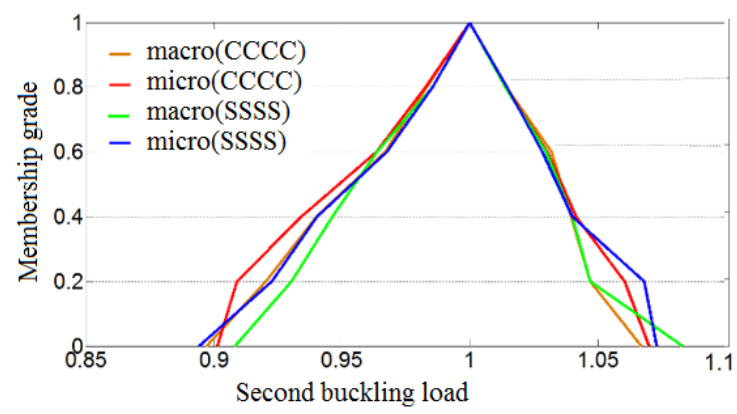

(b)

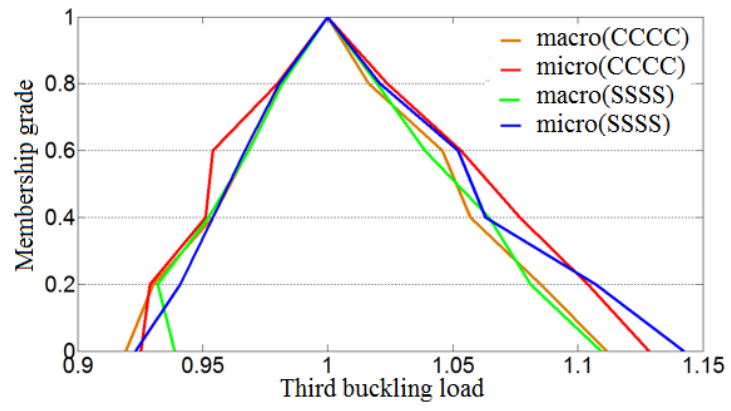

(c)

Fig. 32 Comparative results for buckling loads considering micromechanical $\left(g_{\text {micro }}^{C}\right)$ and macromechanical analyses $\left(g_{\text {macro }}^{C}\right)$ 


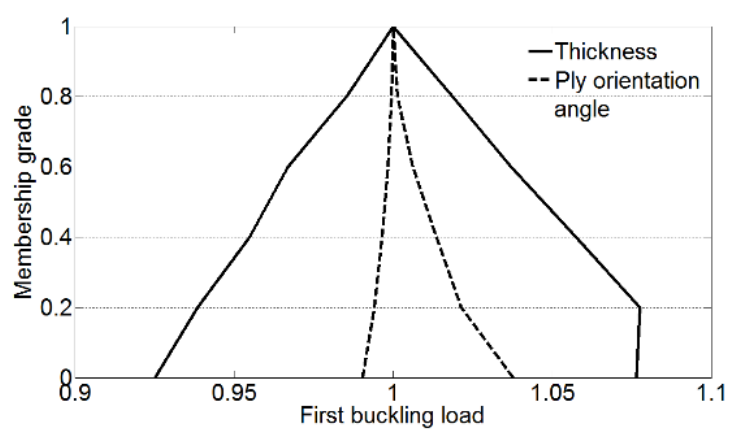

(a)

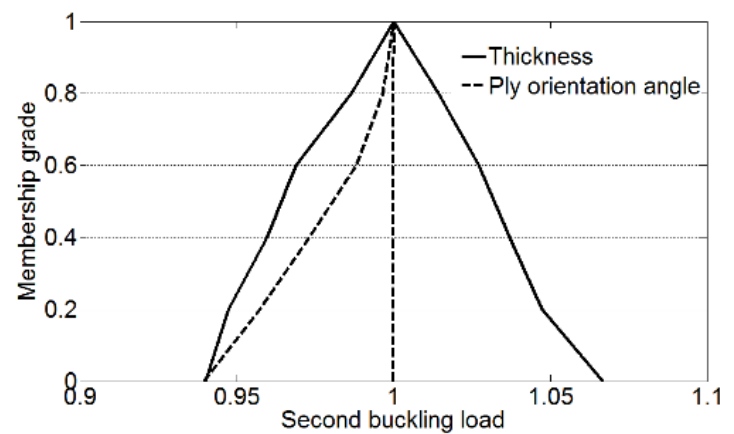

(b)

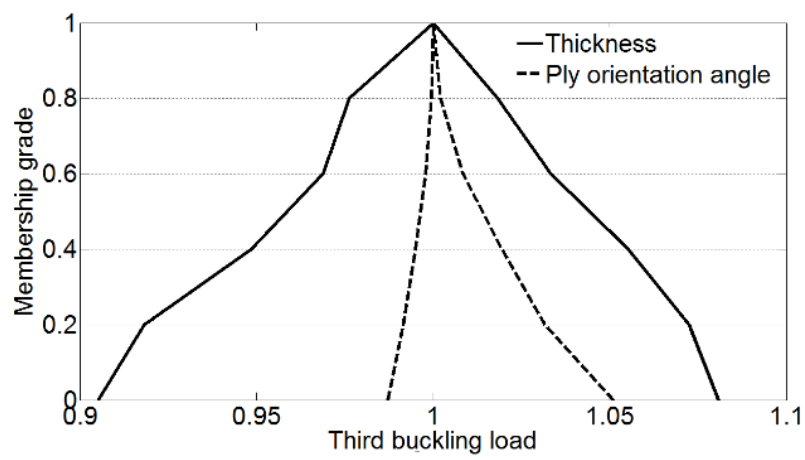

(c)

Fig. 33 Individual effect of fuzzy uncertainty in the geometric properties $\left(g_{G}^{I}\right)$ of composite plates

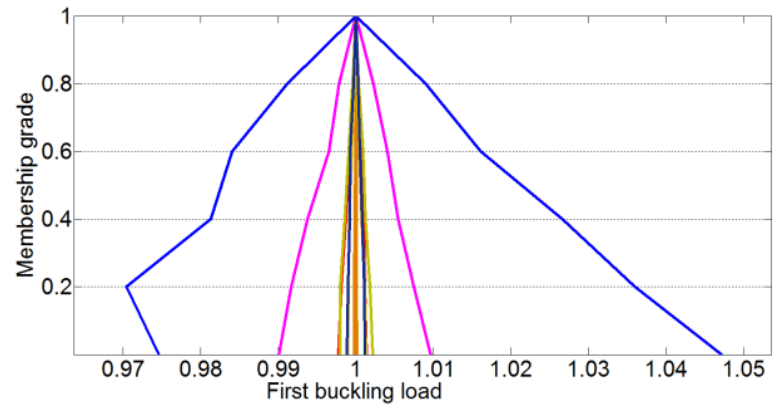

(a)

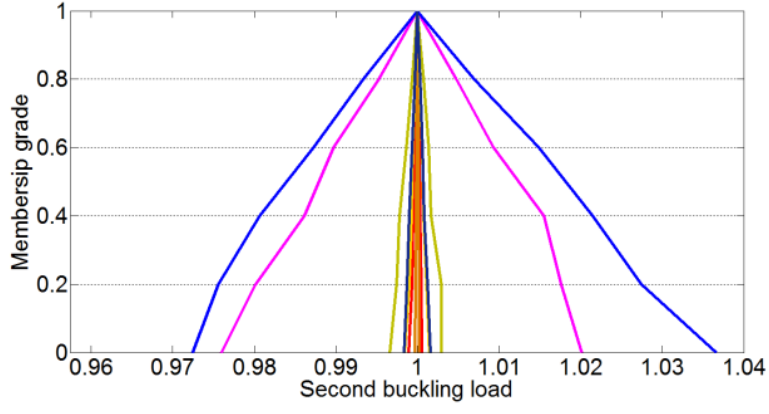

(b)

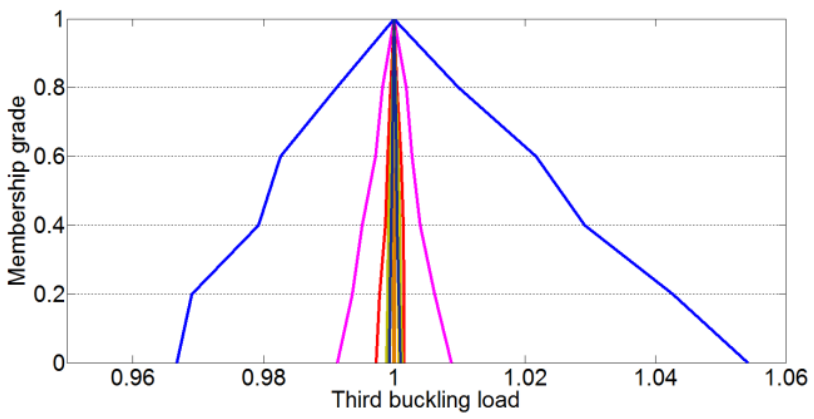

(c)

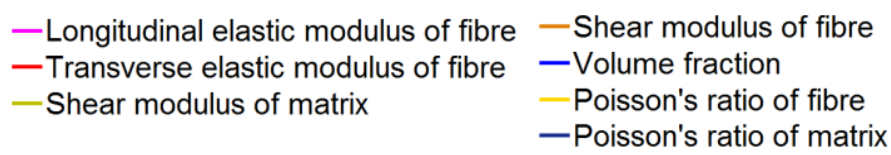

Fig. 34 Individual effect of fuzzy uncertainty in the micromechanical properties $\left(g_{\text {micro }}^{I}\right)$ 


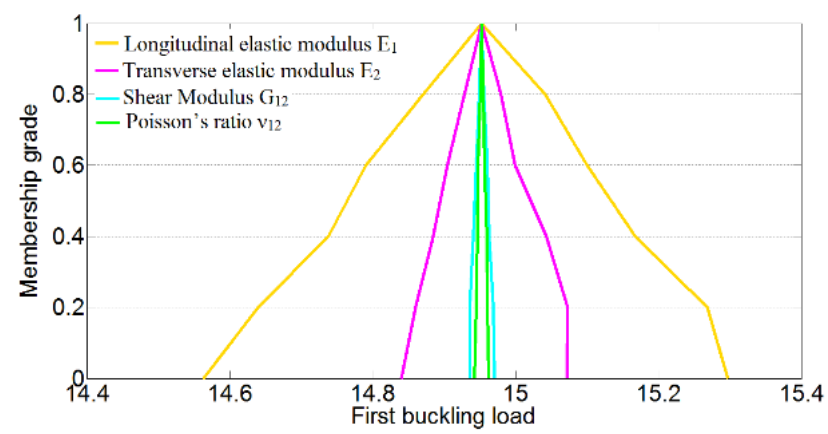

(a)

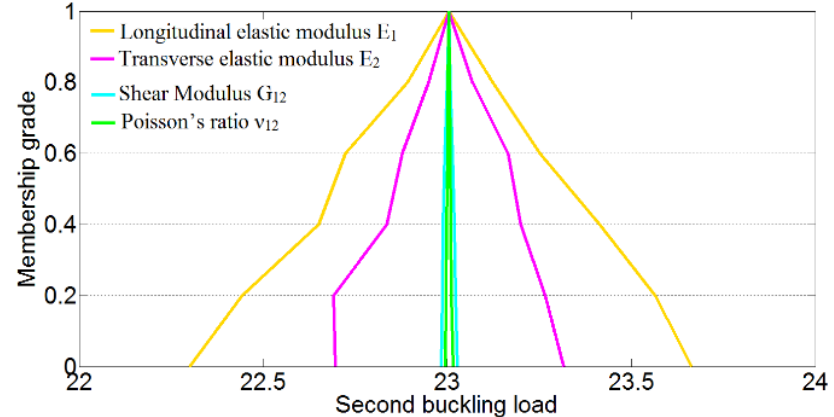

(b)

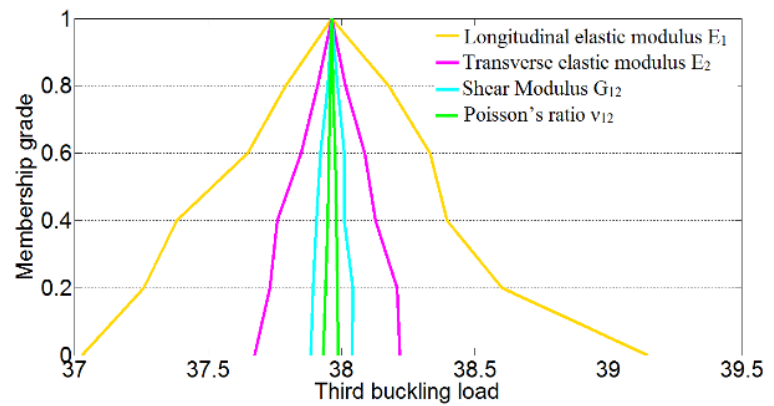

(c)

Fig. 35 Individual effect of fuzzy uncertainty in the macromechanical properties $\left(g_{\text {macro }}^{I}\right)$

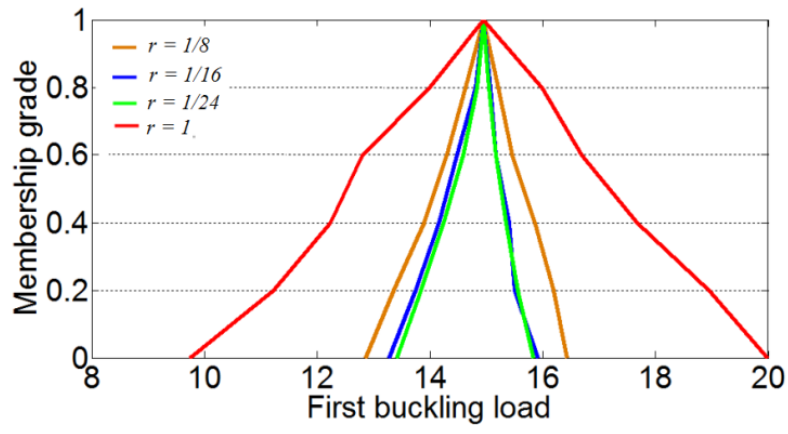

(a)

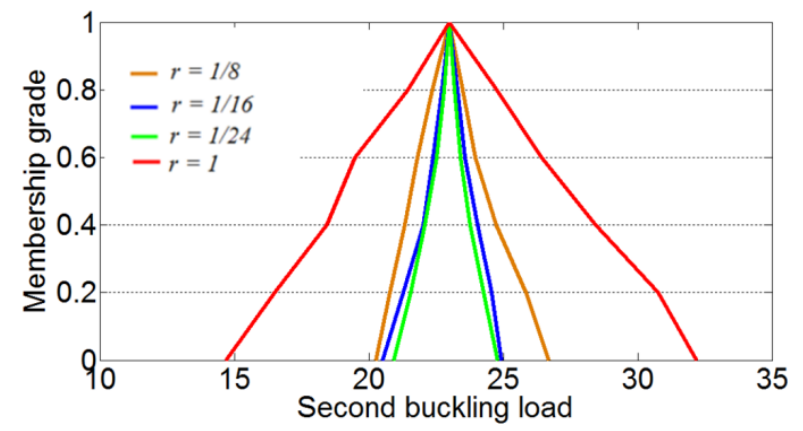

(b)

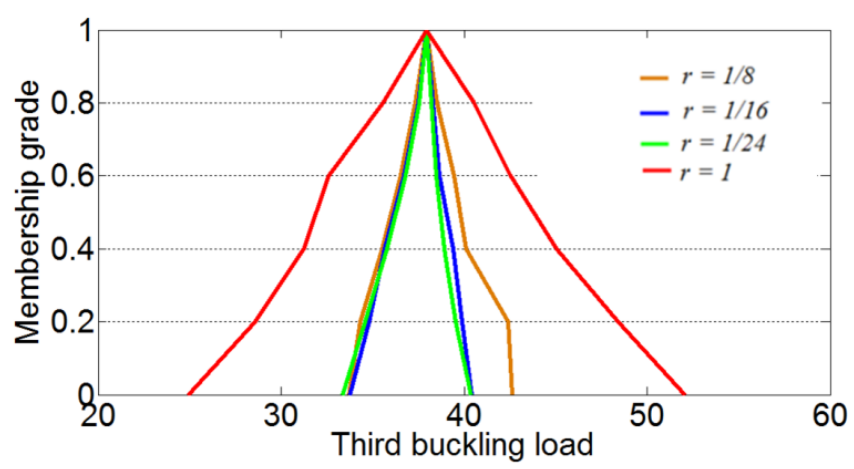

(c)

Fig. 36 Effect of characteristic length $(r)$ on the fuzzy buckling loads ( $g_{\text {micro }}^{C}$ ) 


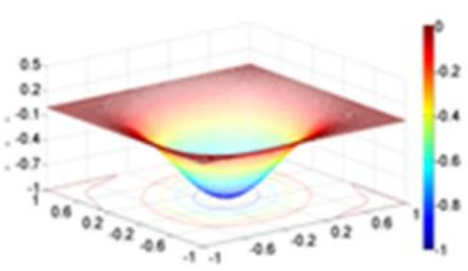

(a)

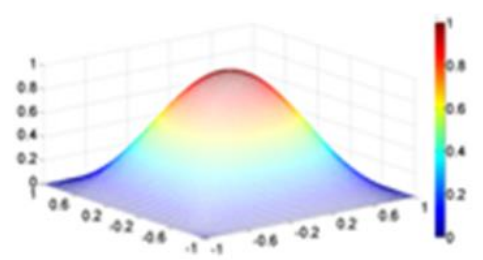

(d)

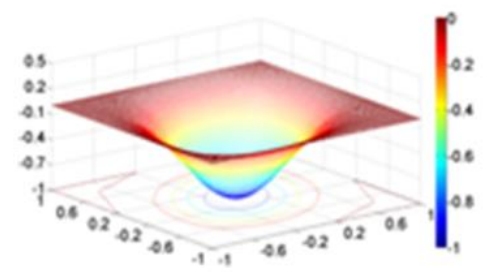

(g)

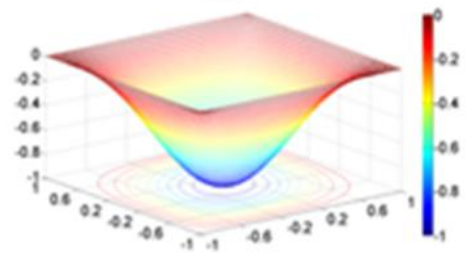

(j)

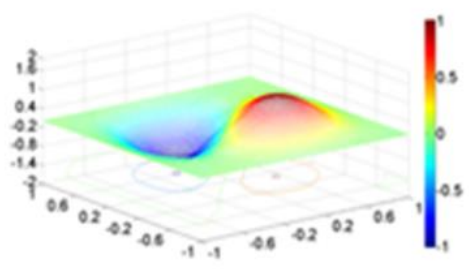

(b)

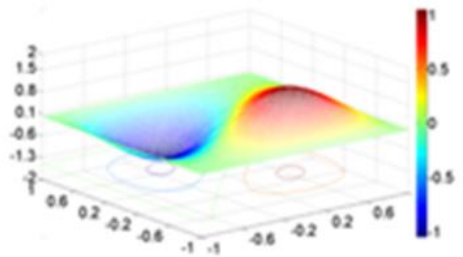

(e)

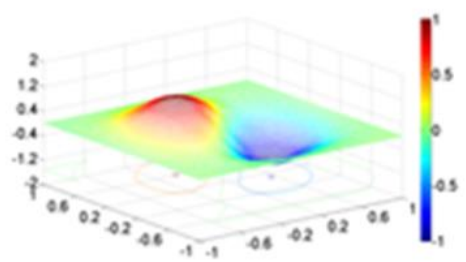

(h)

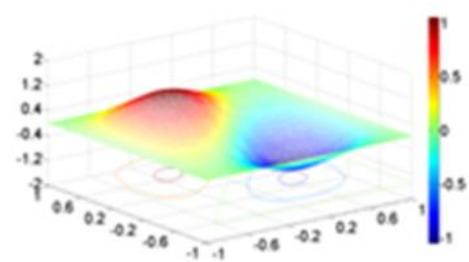

(k)

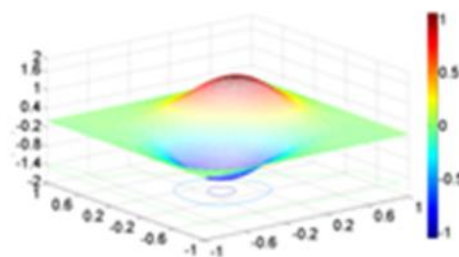

(c)

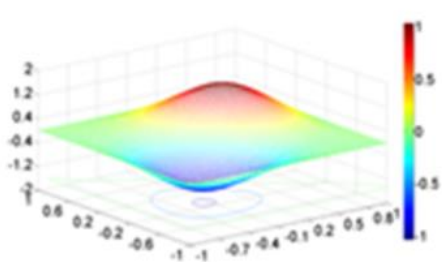

(f)

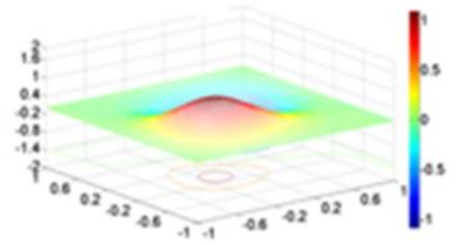

(i)

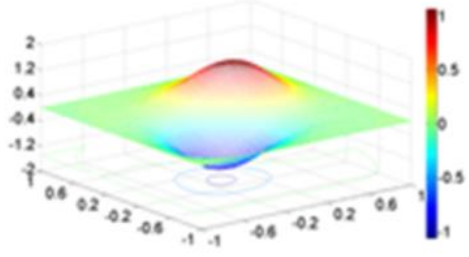

(1)
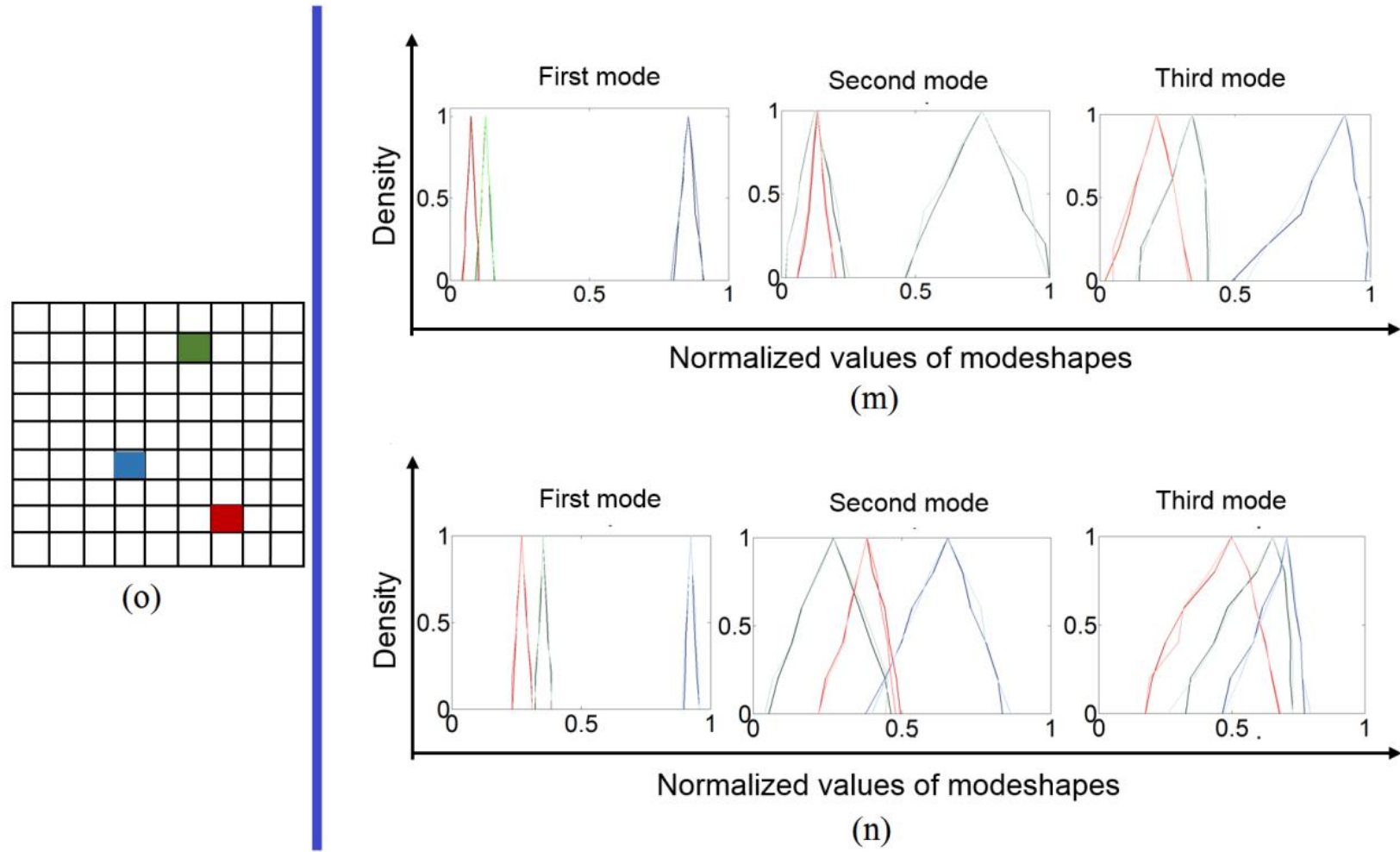

Normalized values of modeshapes

(m)

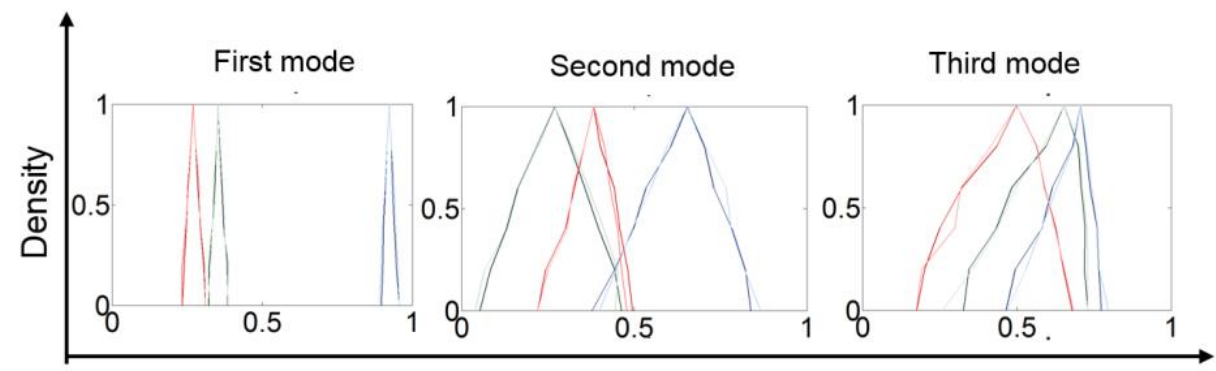

Normalized values of modeshapes

(n)

Fig. 37 Fuzzy modeshapes and representative membership functions of the normalized eigenvectors ( $g_{\text {micro }}^{C}$ and $g_{\text {macro }}^{C}$ ) 
(refer to figure $37(\mathrm{~g}-\mathrm{i})$ ) and a clamped (CCCC) composite plate with stochasticity in the micromechanical $\left(g_{\text {micro }}^{C}\right)$ properties (refer to figure $37(\mathrm{j}-1)$ ). From the mode shapes presented in figure $37(a-1)$, it can be observed that the basic global pattern of the fuzzy mode shapes remains similar to the deterministic case. However, the value of normalized eigenvectors becomes fuzzy in nature for each of the elements in the composite plate. Fuzzy membership functions of the normalized eigenvectors of first three buckling modes for the elements indicated in figure 37(o) are shown considering a clamped (CCCC) boundary condition (refer to figure $30(\mathrm{~m})$ ) and a simply supported (SSSS) boundary condition (refer to figure 37(n)). The results for micro and macro mechanical analyses are shown using lighter and darker shades of respective colours indicated in figure 37(o). It can be noticed that the membership function plots depend significantly on the type of analysis (micro and macro mechanical) and location of the element under consideration.

\section{Summary and perspective}

This paper presents an efficient non-probabilistic bottom-up framework for analyzing the fuzzy dynamics and stabilty of composite laminates with spatially varying system properties. A novel idea of FRVE is proposed in the context of two dimensional plate-like structures to incorporate the spatially varying fuzzy material properties in conjunction with the FE analysis. It can be noted here that the conventional methods of representative volume element (RVE) based analyses and other available analytical solutions can not consider the effect of spatial variability in the system properties. In the proposed FRVE based approach, various other spatially varying system properties (such as varying intensity of matrix cracking, varying fibre properties, fibre breakage etc.) can also be easily accounted following a non-probabilistic framework in future. However, an investigation involving Monte Carlo simulation and finite element analysis becomes exorbitantly computationally expensive. To mitigate this lacuna, a RBF based approach is adopted to achieve computational efficiency (without compromising the accuracy of results) in the present analysis.

In the fuzzy multiscale characterization of composite laminates, there exists three distinct stages of the analysis: fuzzy uncertainty modelling at the input level, propagation of uncertainty to 
the global level and quantification of the global responses such as dynamics and stability characteristics. In most of the studies concerning the fuzzy uncertainty quantification of composite structures, due to simplicity of the approach in implementation, the inevitable spatial variation of the material properties are ignored for any particular realization of Monte Carlo simulation [Dey et al. (2016b)]. However, in reality, the material properties are not constant spatially in a plate-like structure; rather they are spatially varying (as shown in figure 4(c-d)). In the present study, we have adopted a practically relavant spatially vatying non-probabilistic uncertainty modelling strategy. Two separate analyses have been performed considering the source-uncertainty in micro and macro mechanical properties to present a comparative perspective. After modelling the uncertainty in a practically relavant way, the effect of uncertainty needs to be propagated towards the global responses from the elementary input level. The propagation of uncertainty is carried out throgh a stochastic computational model of the structure using a Monte Carlo simulation based minimizationmaximization algorithm. In general, for complex composite laminated structures with spatially varying system properties, the performance functions are not available as explicit functions of the fuzzy input variables. Thus the performance functions or responses (such as natural frequencies and buckling loads) of the structure can only be computed numerically at the end of an intensive structural analysis procedure (such as the FE method), which is often exorbitantly time-consuming and computationally expensive. The surrogate based uncertainty propagation strategy, as adopted in this study, can develop a representative and predictive mathematical/ statistical metamodel relating the natural frequencies and buckling loads to a number of fuzzy input variables. Thereafter the metamodels (response surface) are used to compute the dynamic and stability characteristics corresponding to a given set of fuzzy input variables, instead of having to simulate repeatedly the time-consuming FE analysis. The response surface here represents the results (or outputs) of the structural analyses encompassing (in theory) every prospective combination of the stochastic input variables. Hence, thousands of combinations of the fuzzy input variables can be created and a pseudo analysis (efficient, yet accurate) for each variable set can be performed by adopting the corresponding RBF model. It can be noted in this context that the RBF model is formed only for $\alpha=$ 
0, while the same RBF model can be used for prediction corresponding to the other $\alpha$-cuts. The final step in the fuzzy analysis is uncertainty quantification in the output responses, which is effectively carried out by deriving membership functions for the output quantities of interest.

In case of the fuzzy micro-mechanical analysis, the source-uncertainty is accounted in the micro-mechanical material properties (spatially varying) of composites. Thereafter, the equivalent macro-scale material properties are computed based on the proposed FRVE approach. In the next stage, the equivalent macro-scale material attributes are fed into the finite element code to compute the global responses (like natural frequencies and buckling loads) of the composite plate. Thus, in the fuzzy micromechanical analysis, stochasticity is essentially accounted at the micro-scale level first, and then the effect is propagated to the macro-scale level to characterize the global responses of the structural system. It is common in scientific literature to refer this genre of analysis for composite materials and structures considering micro-mechanical properties based on representative volume element (RVE) as multi-scale analysis [May et al. (2014)]. As fuzzy material and structural parameters are considered in the present analysis leading to a non-probabilistic characterization of the structural responses, the study is referred as fuzzy multi-scale analysis in this paper. It can be noted in this context that there is a possibility of modelling spatial variability of the fibre orientation angle as well. However, we have restricted ourselves with modelling the orientation of the fibres in a straight profile within a random bound (i.e. the orientation of the fibres are stochastic in nature, but it does not vary along the profile in a relative sense). In case of unidirectional fibre reinforced composites, form a manufacturing point of view, the present representation of uncertainty in fiber orientation could be viewed as more practically relevant as the possibility of occurring high degree of spatial randomness in the profile of the fibres is relatively negligible compared to variation in the fiber angle with a straight profile (because the fibres would naturally have a tendency to maintain a straight profile during the manufacturing process due to the inherent stiffness). For this reason, we have adopted a mixed form of uncertainty modelling with spatial variability in the material properties and random fibre orientation angle (meso-scale property) with no relative variation in the direction along the profile of the fibres. 


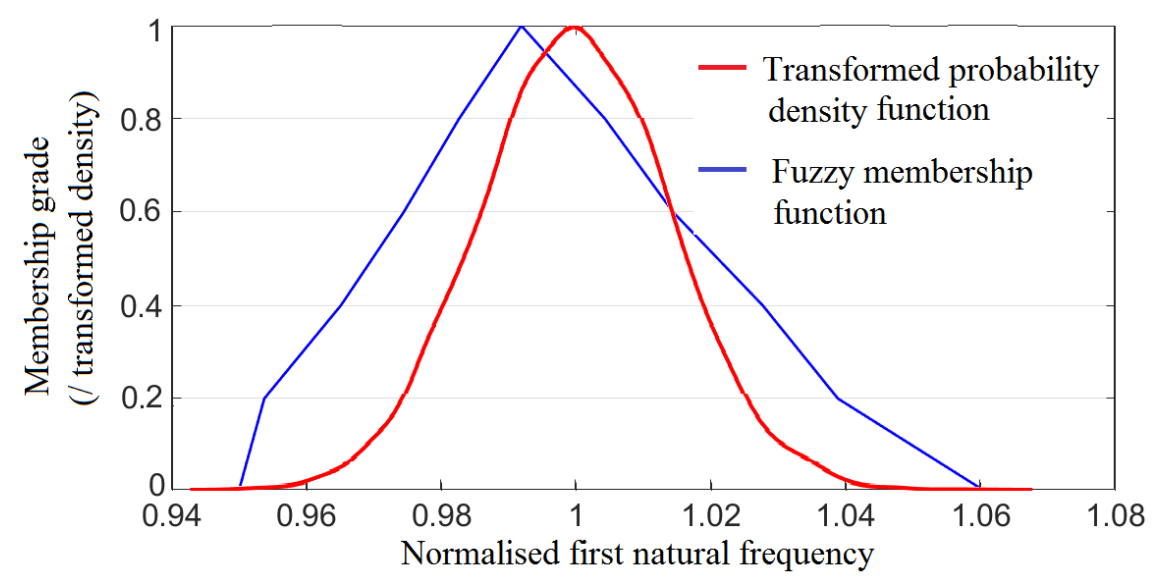

(a)

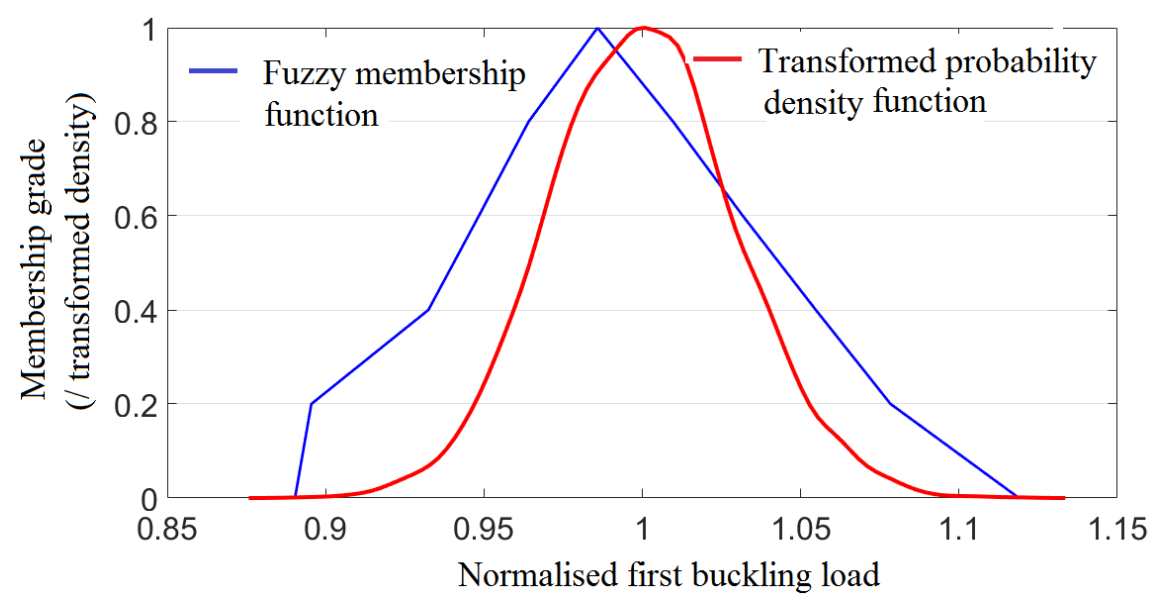

(b)

Fig. 38 A comparative perspective on probabilistic and non-probabilistic analyses considering stochasticity in micromechanical properties $\left(g_{\text {micro }}^{C}\right)$. Here the values of first natural frequency and first buckling load are normalized with respect to the corresponding mean values. The densities for plotting the probability density function plots are normalised with respect to the maximum value of density so that the maximum value of the transformed density becomes 1 for the purpose of providing a comparative perspective with respect to the fuzzy membership function.

No correlation between the material and structural properties are considered in this paper. Normally, establishing the parameters for correlation between the input features (including spatial correlation) requires substantial amount of experimental investigations. The whole point of carrying out a fuzzy analysis being unavailability of such experimental data, we have avoided considering correlation among the input parameters in this study. Moreover, consideration of a typical random field based approach like gaussian or lognormal random fields, may make the current analysis pseudo-probabilistic in nature. These reasons led to our decision of not considering any spatial correlation in this paper on non-probabilistic analysis of composites. The present form of analysis may slightly overestimate the fuzzy response bounds, but that is in the safer side from a design point 
of view. However, it is interesting to notice that the present approach of spatially varying fuzzy uncertainty modelling leads to a significantly reduced fuzzy response bound compared to the conventional approach of modelling without such spatial variation (such as Dey et al. 2016b).

Oftentimes designers like to create a design factor or factor of safety out of the probability analysis of structures. A fuzzy analysis can lead to evaluating the safety factor of design in the nonprobabilistic regime. In fact, a fuzzy based analysis could give us the opportunity to define the fuzzy factor of safety (FFoS) corresponding to different alpha-cut levels. Normally, factor of safety (FoS) for a design parameter is defined as: $\mathrm{FoS}=$ (Capacity of the system in terms of the design parameter / Maximum allowable value of the design parameter). In case of fuzzy analysis, we have a bound (defined by the upper and lower values) corresponding to each of the alpha-cuts. Thus a bound of the factor of safety can be defined in case of the fuzzy analysis instead of a single value. Let us consider, $p_{i}^{(j, L)}$ and $p_{i}^{(j, U)}$ denote the lower and upper bounds of a fuzzy interval (the superscripts $L$ and $U$ are used to denote the lower and upper bounds respectively) at the $j$-th level of $\alpha$-cut for a certain parameter $i$. The design value of that parameter is given by $p_{i}^{D}$. The bound of FFoS can be defined as $\left[p_{i}^{(j, L)} / p_{i}^{D}, p_{i}^{(j, U)} / p_{i}^{D}\right]$ for the $j$-th level of $\alpha$-cut. It could be noted that the concept of FFoS brings the notion of defining the level of safety corresponding to a particular degree of fuzziness. Thus it would provide a clear perspective on how much the factor of safety could vary (in terms of a bound) in case of a stochastic design for various degree of uncertainty (i.e. corresponding to different $\alpha$-cuts).

From an engineering perspective, the idea of a non-probabilistic fuzzy based analysis and a probabilistic analysis is quite straight-forward. In a probabilistic analysis, the complete nature of statistical distributions for the input parameters is known and this leads to obtaining the complete probability distribution of the output quantities including the upper and lower bounds. In case of a fuzzy based analysis, the statistical distributions of the input parameters are not known; rather an idea about the mean value of the input parameters exists. The membership functions are defined for the input parameters to establish lower and upper bounds corresponding to different degree of 
fuzziness. Subsequently, the uncertainty in the output parameters is quantified in terms of lower and upper bounds corresponding to different degree of fuzziness. The degree of fuzziness is analogous to the level of uncertainty associated with a parameter. As a fuzzy analysis provides only the bounds of output parameters instead of a complete probabilistic description between the bounds, the output of a fuzzy analysis can be regarded as a subset of probabilistic analysis. It can be noted that the lower and upper bounds of a parameter in the probability distribution coincide with the bounds of a membership function corresponding to $\alpha=0$. The fascinating idea of combining the interval analyses for different degree of fuzziness to form a membership function gives more information than the just two bounds of the output parameters. Engineers, often being more interested in the range of variability, a fuzzy analysis caters to this requirement quite well along with an additional factor of the level of uncertainty (i.e. range of variability of the parameter corresponding to degree of fuzziness). To provide a comparative perspective, figure 38 shows transformed probability density function plots and membership function plots for a micromechanical analysis involving the first natural frequency and first buckling load, wherein all the above-mentioned features become quite evident. As the debate on 'fuzziness is probability in disguise' will continue; in essence, a fuzzy based approach is capable of providing the engineers a way to comprehensively quantify uncertainty in the absence of statistical distributions of the input parameters.

\section{Conclusion}

A bottom-up non-probabilistic analysis is presented in this article for multi-scale quantification of the effect of fuzzy source-uncertainty in the dynamics and stability behaviour of composite plates. A practically relevant non-probabilistic uncertainty modelling approach is proposed considering spatially varying system attributes, for which a novel concept of fuzzy representative volume element (FRVE) is developed. The non-probabilistic approach (such as fuzzy uncertainty), as presented in this paper, can allow us to characterize the effect of uncertainty in a system when the statistical distributions of the input parameters remain unavailable due to the restriction of performing a large number of experiments. A non-linear and non-monotonic relationship between the output quantities and the input parameters is evident in the fuzzy analysis in case of multiple numerical 
results. The paper reveals that the conventional practise of neglecting the inevitable spatial variation in material properties over-estimates the fuzzy response bounds of the natural frequencies and buckling loads. Such outcome will have an influential impact on the current design procedures in the non-probabilistic approach. Besides an in-depth analysis of fuzzy natural frequencies and buckling loads, the effect of fuzzy uncertainty is investigated on the mode shapes of composite plates. The fuzzy response bounds also provide a relative sense of sensitivity to the uncertainty in different results presented in this paper. To understand the relative influence of different input parameters, the individual effects of fuzzy uncertainty are analysed considering micro and macro mechanical properties. For achieving computational efficiency, a RBF based stochastic analysis algorithm is developed in conjunction with the fuzzy finite element model, wherein it is noted that the required number of original FE simulations can be remarkably reduced without compromising accuracy of results.

Even in the absence of adequate statistical information of the stochastic input parameters, the fuzzy stochasticity in material/ structural attributes is found to influence the system performance significantly depending on the degree of fuzziness, affirming the necessity to consider such forms of source-uncertainties during the analysis to ensure adequate safety, sustainability and robustness of the structure. Novelty of this paper includes the consideration of spatial variation in material properties (micro/ macro) following the FRVE approach coupled with the RBF algorithm in context to composite laminates. The idea of FRVE is quite generic in nature; this concept can be extended to other structures and various other non-probabilistic systems with spatial variability in two/ three dimensions.

\section{Acknowledgements}

SN and SS are grateful for the support provided through the Lloyd's Register Foundation Centre. The Foundation helps to protect life and property by supporting engineering-related education, public engagement and the application of research. 


\section{References}

Adhikari S., Haddad Khodaparast H., (2014) A spectral approach for fuzzy uncertainty propagation in finite element analysis, Fuzzy Sets and Systems, 243, 1-24.

Altmann F., Sickert J.U., Mechtcherine V., Kaliske M., (2012) A fuzzy-probabilistic durability concept for strain-hardening cement-based composites (SHCCs) exposed to chlorides: Part 1: Concept development, Cement and Concrete Composites, 34(6), 754-762.

Babuška Ivo, Silva Renato S., (2014) Dealing with uncertainties in engineering problems using only available data, Computer Methods in Applied Mechanics and Engineering, 270, 57-75.

Bathe K.J., (1990) Finite Element Procedures in Engineering Analysis, PHI, New Delhi.

Beatson R K, Fast Fitting of Radial Basis Functions: Methods Based on Preconditioned gmres Iteration, Advances in Computational Mathematics, 11 253-270, 1999

Chakrabarti A, Sheikh A H, Griffith M, Oehlers D J, Dynamic Response of composite beams with partial shear interactions using a higher order beam theory, Journal of Structural Engineering, 139 (1) 47-56, 2013.

Chandrashekhar M, Ganguli R. Damage Assessment of structures with uncertainty in curvature mode shapes using Fuzzy Logic. Journal of Sound and Vibration. 2009;326(3-5)

Chowdhury R., Adhikari S., (2012) Fuzzy parametric uncertainty analysis of linear dynamical systems: A surrogate modeling approach, Mech. Syst. Signal Process., 32(10), 5-17.

Degrauwe D., Lombaert G., Roeck G.D., (2010) Improving interval analysis in finite element calculations by means of affine arithmetic, Comput. Struct., 88(3-4), 247-254.

Denga Yong, Chen Yuxin, Zhang Yajuan, Mahadevan Sankaran, (2012) Fuzzy Dijkstra algorithm for shortest path problem under uncertain environment, Applied Soft Computing , 12(3), 12311237.

Dey S, Mukhopadhyay $\mathrm{T}$, Khodaparast $\mathrm{H} \mathrm{H}$, Adhikari S, Fuzzy uncertainty propagation in composites using Gram-Schmidt polynomial chaos expansion, Applied Mathematical Modelling, 40 (7-8) 4412-4428, 2016b.

Dey S, Mukhopadhyay T, Naskar S, Dey T K, Chalak H D, Adhikari S, Probabilistic characterization for dynamics and stability of laminated soft core sandwich plates, Journal of Sandwich Structures \& Materials, 2018, DOI: 10.1177/1099636217694229.

Dey S, Mukhopadhyay T, Spickenheuer A, Gohs U, Adhikari S, Uncertainty quantification in natural frequency of composite plates - An Artificial neural network based approach, Advanced Composites Letters, 25(2) 43-48, 2016 a.

Dey S., Mukhopadhyay T., Adhikari S. (2018a) Uncertainty quantification in laminated composites: A meta-model based approach, CRC Press, ISBN 9781498784450 
Dey S., Mukhopadhyay T., Khodaparast H. H., Adhikari S. (2016d) A response surface modelling approach for resonance driven reliability based optimization of composite shells, Periodica Polytechnica - Civil Engineering, 60 (1) 103-111

Dey S., Mukhopadhyay T., Sahu S. K., Adhikari S. (2018b) Stochastic dynamic stability analysis of composite curved panels subjected to non-uniform partial edge loading, European Journal of Mechanics / A Solids, 67 108-122

Dey S., Mukhopadhyay T., Spickenheuer A., Adhikari S., Heinrich G. (2016c) Bottom up surrogate based approach for stochastic frequency response analysis of laminated composite plates, Composite Structures, 140 712-727

Dey S., Naskar S., Mukhopadhyay T., Gohs U., Sriramula S., Adhikari S., Heinrich G. (2016e) Uncertain natural frequency analysis of composite plates including effect of noise - A polynomial neural network approach, Composite Structures, 143 130-142

Dey T.K., Mukhopadhyay T., Chakrabarti A., Sharma U.K.(2015) Efficient lightweight design of FRP bridge deck, Proceedings of the Institution of Civil Engineers - Structures and Buildings, 168 (10) $697-707$

Díaz-Madroñero M., Peidro D., Mula J., (2014) A fuzzy optimization approach for procurement transport operational planning in an automobile supply chain, Applied Mathematical Modelling, 38(23), 5705-5725.

Fasshauer G E, Solving Partial Differential Equations by Collocation with Radial Basis Functions, Surface Fitting and Multiresolution Methods, Vol. 2, Proceedings of the 3rd International Conference on Curves and Surfaces, 2 131-138, 1997

Hanss M., Willner K., (2000) A fuzzy arithmetical approach to the solution of finite element problems with uncertain parameters, Mech.Research Comm., 27(3), 257-272.

Hon Y C, Mao X Z, On Unsymmetric Collocation by Radial Basis Functions, Applied. Math. Computation., 119 177-186, 2001

Jones R M, Mechanics of Composite structures, Taylor \& Francis, Philadelphia, PA, 1999.

Kansa E J, Hon Y C, Circunvecting the Ill-Conditioning Problem with Multiquadric Radial Basis Functions, Comput. Math. Appl., 39 123-137, 2000

Kansa E J, Multiquadrics: A Scattered Data Approximation Scheme with Applications to Computational Fluid Dynamics. I: Surface Approximations and Partial Derivative Estimates, Comput. Math. Appl., 19 127-145, 1990a

Kansa E J, Multiquadrics: A Scattered Data Approximation Scheme with Applications to Computational Fluid Dynamics. II: Solutions to Parabolic, Hyperbolic and Elliptic Partial Differential Equations, Comput. Math. Appl., 19 147-161, 1990b

Karsh P K, Mukhopadhyay T, Dey S, Spatial vulnerability analysis for the first ply failure strength of composite laminates including effect of delamination, Composite Structures, 184 554-567, $2018 \mathrm{a}$. 
Karsh P. K., Mukhopadhyay T., Dey S. (2018b) Stochastic dynamic analysis of twisted functionally graded plates, Composites Part B: Engineering, 147 259-278

Krishnamurthy, T., Response surface approximation with augmented and compactly supported radial basis functions, The 44th AIAA/ASME/ASCE/AHS/ASC structures, structural dynamics, and materials conference, Norfolk, VA, 2003

Lal A, Singh B N, Stochastic Free Vibration of Laminated Composite Plates in Thermal Environments, Journal of Thermoplastic Composite Materials, 23(1) 57-77, 2010.

Lei Z., Chen Q., (2002) A new approach to fuzzy finite element analysis, Computer Methods in Applied Mechanics and Engineering, 191(45), 5113-5118.

Liew K M, Huang Y Q, Bending and buckling of thick symmetric rectangular laminates using the moving least-squares differential quadrature method, International Journal of Mechanical Sciences, 45 95-114, 2003.

Liew K. M., Solving the vibration of thick symmetric laminates by reissner/mindlin plate theory and the p-ritz method, Journal of Sound and Vibration, 198(3) 343-360, 1996

Mace B., Worden K., Manson G., (2005) Uncertainty in structural dynamics, J. Sound Vib., 288(3), 423-429.

Maharshi K., Mukhopadhyay T., Roy B., Roy L., Dey S. (2018) Stochastic dynamic behaviour of hydrodynamic journal bearings including the effect of surface roughness, International Journal of Mechanical Sciences, 142-143 370-383

Mahata A., Mukhopadhyay T. (2018) Probing the chirality-dependent elastic properties and crack propagation behavior of single and bilayer stanene, Physical Chemistry Chemical Physics, 20 $22768-22782$

Mahata A., Mukhopadhyay T., Adhikari S. (2016) A polynomial chaos expansion based molecular dynamics study for probabilistic strength analysis of nano-twinned copper, Materials Research Express, 3036501

Manan A, Cooper J, Design of composite wings including uncertainties: a probabilistic approach, $J$ Aircr, 46(2) 601-7, 2009.

May M, Nossek M, Petrinic N, Hiermaier S, Thoma K, Adaptive multi-scale modeling of high velocity impact on composite panels, Composites: Part A, 58 56-64, 2014.

Metya S., Mukhopadhyay T., Adhikari S., Bhattacharya G. (2017) System Reliability Analysis of Soil Slopes with General Slip Surfaces Using Multivariate Adaptive Regression Splines, Computers and Geotechnics, 87 212-228

Moens D., Hanss M., (2011) Non-probabilistic finite element analysis for parametric uncertainty treatment in applied mechanics: Recent advances, Finite Elements in Analysis and Design, 47(1), $4-16$. 
Moens D., Vandepitte D. Recent advances in non-probabilistic approaches for non-deterministic dynamic finite element analysis. Archives of Computational Methods in Engineering, 13(3):389-464, 2006

Moens, D., Vandepitte, D., A survey of non-probabilistic uncertainty treatment in finite element analysis. Computer methods in applied mechanics and engineering, 194(12-16), 1527-1555, 2005

Möller, B., Beer Michael, Fuzzy Randomness - Uncertainty in Civil Engineering and Computational Mechanics, Springer, Berlin, 2004.

Moore R.E., Interval Analysis, Prentice-Hall, Englewood Cliffs, NJ, USA, 1966.

Muc A., Kędziora P, (2001) A fuzzy set analysis for a fracture and fatigue damage response of composite materials, Composite Structures, 54(2-3), 283-287.

Mukhopadhyay T, Adhikari S, Effective in-plane elastic moduli of quasi-random spatially irregular hexagonal lattices, International Journal of Engineering Science, 119 142-179, 2017a.

Mukhopadhyay T, Adhikari S, Equivalent in-plane elastic properties of irregular honeycombs: An analytical approach, International Journal of Solids and Structures, 91 169-184, 2016a.

Mukhopadhyay T, Adhikari S, Stochastic mechanics of metamaterials, Composite Structures, 162 85-97, 2017b.

Mukhopadhyay T., Mahata A., Dey S., Adhikari S. (2016b) Probabilistic analysis and design of HCP nanowires: an efficient surrogate based molecular dynamics simulation approach, Journal of Materials Science \& Technology, 32(12) 1345-1351

Mukhopadhyay T., A multivariate adaptive regression splines based damage identification methodology for web core composite bridges including the effect of noise, Journal of Sandwich Structures \& Materials, 2018, DOI: 10.1177/1099636216682533

Mukhopadhyay T., Adhikari S. (2016b) Free vibration analysis of sandwich panels with randomly irregular honeycomb core, Journal of Engineering Mechanics, 142(11) 06016008

Mukhopadhyay T., Adhikari S., Batou A., Frequency domain homogenization for the viscoelastic properties of spatially correlated quasi-periodic lattices, International Journal of Mechanical Sciences, 2018b, DOI: 10.1016/j.ijmecsci.2017.09.004

Mukhopadhyay T., Chowdhury R., Chakrabarti A. (2016a) Structural damage identification: A random sampling-high dimensional model representation approach, Advances in Structural Engineering, 19(6) 908-927

Mukhopadhyay T., Dey T. K.,Chowdhury R., Chakrabarti A., Adhikari S. (2015) Optimum design of FRP bridge deck: an efficient RS-HDMR based approach, Structural and Multidisciplinary Optimization, 52 (3) 459-477

Mukhopadhyay T., Mahata A., Adhikari S., Asle Zaeem M. (2018a) Probing the shear modulus of two-dimensional multiplanar nanostructures and heterostructures, Nanoscale, 105280 - 5294

Mukhopadhyay T., Mahata A., Adhikari S., Asle Zaeem M. (2017) Effective mechanical properties of multilayer nano-heterostructures, Nature Scientific Reports, 715818 
Mukhopadhyay T., Naskar S., Dey S., Adhikari S. (2016c) On quantifying the effect of noise in surrogate based stochastic free vibration analysis of laminated composite shallow shells, Composite Structures, 140 798-805

Naskar S, Mukhopadhyay T, Sriramula S, Adhikari, Stochastic natural frequency analysis of damaged thin-walled laminated composite beams with uncertainty in micromechanical properties, Composite Structure, 160 312-334, 2017a

Naskar S., Mukhopadhyay T., Sriramula S. Probabilistic micromechanical spatial variability quantification in laminated composites, Composites Part B: Engineering, 151 291-325, 2018

Naskar, S. \& Sriramula, S. (2017a). 'Random Field Based Approach for Quantifying the Spatial Variability in Composite Laminates'. Paper presented at 20th International Conference on Composite Structures (ICCS20), paris, France, 4/09/17 - 7/09/17.

Naskar, S. \& Sriramula, S. (2017b). 'Vibration analysis of hollow circular laminated composite beams - A stochastic approach'. Paper presented at 12th International Conference on Structural Safety \& Reliability, Vienna, Austria, 6/08/17 - 10/08/17.

Naskar, S. \& Sriramula, S. (2017c). 'Effective Elastic Property of Randomly Damaged Composite Laminates'. Engineering Postgraduate Research Symposium, Aberdeen, United Kingdom, 26/04/17 - 26/04/17.

Naskar, S. \& Sriramula, S. (2018). 'On Quantifying the Effect of Noise in Radial Basis Based Stochastic Free Vibration Analysis of Laminated Composite Beam'. Paper presented at 18 European Conference on Composite Materials, 25/06/18 - 28/06/18

Naskar, S., Mukhopadhyay, T. \& Sriramula, S. (2017b). 'Non-probabilistic analysis of laminated composites based on fuzzy uncertainty quantification'. Paper presented at 20th International Conference on Composite Structures (ICCS20), paris, France, 4/09/17 - 7/09/17.

Neves A M A, Ferreira A J M, Free vibrations and buckling analysis of laminated plates by oscillatory radial basis functions, Curved and Layered Structures, 3(1) 17-21, 2016.

Pawar P M, Nam Jung S., Ronge B P, Fuzzy approach for uncertainty analysis of thin walled composite beams, Aircraft Engineering and Aerospace Technology, 84 (1) 13-22, 2012.

Pawar P, Ganguli R. Fuzzy Logic Based Health Monitoring and Residual Life Prediction of Composite Helicopter Rotor. Journal of Aircraft. 2007; 44(3) 981-995

Powell M J D, Radial basis functions for multivariable interpolation: a review, Algorithms for Approximation, Mason J.C., Cox M.G. (eds.), London, Oxford University Press, 1987

Qiu Z., Wang X., Friswell M., Eigenvalue bounds of structures with uncertain-but-bounded parameters, J. Sound Vib., 282(1-2), (2005), 297-312.

Rao S.S., Annamdas K.K., (2008) Evidence-based fuzzy approach for the safety analysis of uncertain systems, AIAA Journal, 46(9), 2383-2387.

Rayleigh J W, Theory of Sound, Dover Publications, New York, re-issue, second edition, 1945. 
Reddy J. N. (2003) Mechanics of Laminated Composite Plates and Shells: Theory and Analysis, Second Edition, ISBN: 9780849315923

Scarth, C, Adhikari, S, Modelling spatially varying uncertainty in composite structures using lamination parameters, AIAA Journal, 55(11) 3951-3965, 2017.

Shaw, A., Sriramula, S., Gosling, P. D., Chryssanthopoulos, M. K. (2010). A critical reliability evaluation of fibre reinforced composite materials based on probabilistic micro and macromechanical analysis. Composites Part B: Engineering, 41(6), 446-453

Sobol I M, On the distribution of points in a cube and the approximate evaluation of integrals, USSR Comput Math Math Phys, 7 86-112, 1967.

Soden P D, Hinton M J, Kaddour A S, Lamina properties, lay-up configurations and loading conditions for a range of fibre reinforced composite laminates, Compos. Sci. Technol., 58 (7) 1011$1022,1998$.

Sriramula S, Chryssanthopoulos K M, Quantification of uncertainty modelling in stochastic analysis of FRP composites, Composites Part A: Applied Science and Manufacturing, 40 1673-1684, 2009

Tornabene F., Fantuzzi N., Bacciocchi M. (2017). A new doubly-curved shell element for the free vibrations of arbitrarily shaped laminated structures based on Weak Formulation IsoGeometric Analysis. Composite Structures, 171, 429-461

Tornabene F., Fantuzzi N., Bacciocchi M., Viola E. (2018). Mechanical behavior of damaged laminated composites plates and shells: Higher-order Shear Deformation Theories. Composite Structures, 189, 304-329.

Umesh K, Ganguli R, Material uncertainty effect on vibration control of smart composite plate using polynomial chaos expansion, Mech Adv Mater Struct, 20 (7) 580-591, 2013.

Zadeh L.A., (1975) Concept of a linguistic variable and its application to approximate reasoning-I, Information Sciences, 8(3), 199-249.

Zhou, X. Y., Gosling, P. D., Ullah, Z., Kaczmarczyk, L., Pearce, C. J., Stochastic multi-scale finite element based reliability analysis for laminated composite structures. Applied Mathematical Modelling, 45 457-473, 2017 